\title{
SUBMICRON AND NANO SURFACE PATTERNING USING NANOSECOND LASER TECHNIQUE
}

\author{
by \\ Ali Abdul-Kadhum Husein \\ B.Sc., University of Technology (Iraq), 1990

\begin{abstract}
A Thesis
Presented to

Ryerson University

In partial fulfillment of the requirement for the degree of

Master of Applied Science

in
\end{abstract}

The Program of Mechanical Engineering

Toronto, Ontario, Canada

Ali AK. Husein (C 2009 


\section{AUTHOR'S DECLARATION}

I hereby declare that I am the sole author of this thesis report.

I authorize Ryerson University to lend this thesis to other institutions or individuals for the purpose of scholarly research.

\section{Ali AK. Husein \\ Department of Mechanical and Industrial Engineering Ryerson University}

I further authorize Ryerson University to reproduce this project by photocopying or by other means, in total or in part, at the request of other institutions or individuals for the purpose of scholarly research.

\footnotetext{
Ali AK. Husein

Department of Mechanical and Industrial Engineering Ryerson University
} 


\title{
Submicron and Nano Surface Patterning Using Nanosecond Laser Technique
}

\author{
Ali AK. Husein, Master of Applied Science, 2009 \\ Mechanical Engineering, Ryerson University
}

\section{Abstract}

Direct writing by laser techniques in the micro and nanostructuring scale is very important for the fabrication of new materials and multifunctional devices. They have proven to be very successful tools for precision machining and microfabrication with applications in optical devices, microelectronics, medical device, biomedical, defense applications, and MEMS. Focused nanosecond (ns) laser pulses can produce periodic structures and arrays pattern structures in semiconductors and thin metallic film on shaped surfaces. The achievable structure size is restricted by the wavelength and diffraction limit as well as it is determined by material properties and laser pulse stability.

This thesis proposes a nanosecond laser nanostructuring technique in common optical path configuration to examine the limitations of the currently used fabrication methods and type of setups used; the competitive edge is using nanosecond lasers as a tool. Prospectively, this technology can be applied for femtosecond laser fabrication, because this is an easy, simple and common optical path configuration. For this experimental setup, the use of a common optical path configuration for automatic interference offers equals path lengths. It is not required for complicated optical setups while in femtosecond laser setups, it is extremely important to use path compensation in order to offer time delay for one laser beam due to a long path and more optical components. A low repetition rate, low power nanosecond laser system is investigated to 
preventing the (HAZ) conditions. The influence of the laser repetition rate and pulse energy on the size and quality of submicron features which fabricated on silicon wafers and thin gold film is investigated. In terms of nanomachining below the ablation threshold (surface patterning), the influence of laser fluence, repetition rate and pulse energy on the spacing as well as diameter of dots created on silicon wafer surface is examined. These studies show the capability of the proposed system of nanosecond laser in common optical path configuration in meeting the industry requirements. 


\section{Acknowledgments}

I would like to express my deep gratitude and respect to my supervisors, Dr. K. Venkatakrishnan and Dr. Bo Tan, for their fruitful and constructive guidance through the difficult paths of my MASc study. Their scientific and spiritual support was a pole of stability and encouragement during hard times. I am indebted to them for their penetrating guidance as well as their cooperation was very impressive for two years.

I would like to thank Dr. Greg Kawall, Director of Mechanical Engineering Graduate program, all the faculty members, technical officers and administrative staff members for their support and cooperation all the time during my stay at Ryerson University.

I am also grateful to my parents and family for their continued support, prayers and love. Deepest gratitude to my wife and two lovely kids, Sukaina and Mohammad, for their loving care, support, and bringing happiness to my life.

Finally, I am grateful to the Grace of Allah for the countless blessings I have received. 


\section{Table of Contents}

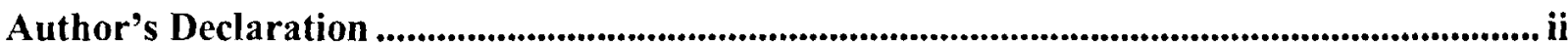

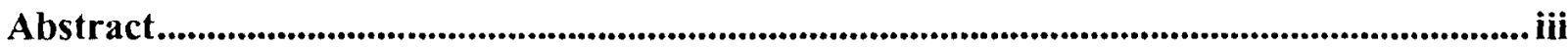

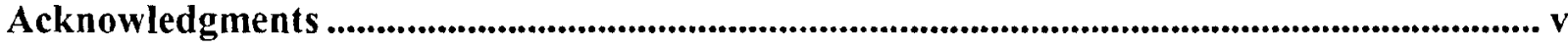

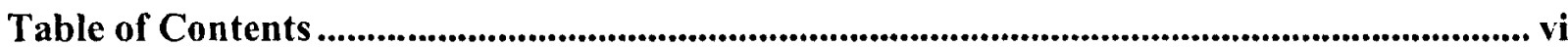

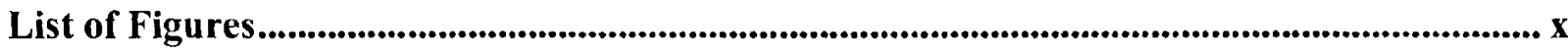

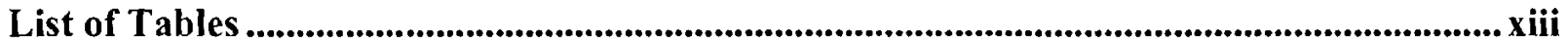

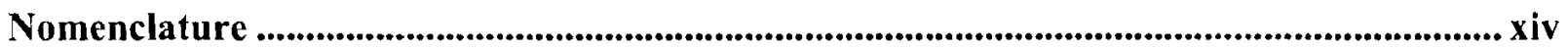

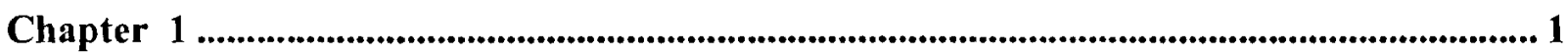

Introduction ............................................................................................................... 1

1.1 Introduction to Submicron and Nano Surface Patterning Techniques ...................... 1

1.1.1 Mechanical Grooves Scribing ............................................................. 2

1.1.2 Contact / Proximity Masking Fabrication Technique .................................. 5

1.1.3 Photolithographic Patterning............................................................... 7

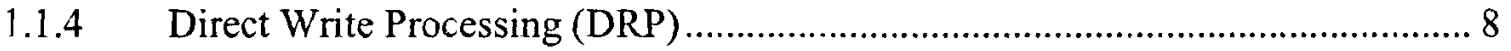

1.1.5 Photochemical Formation ................................................................... 11

1.1.6 Patterning Use Femtosecond Laser..................................................... 12

1.2 Summary of Submicron and Nano Structuring Techniques ................................ 12

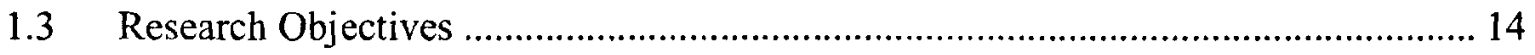

1.4 Overview of Thesis.............................................................................. 15

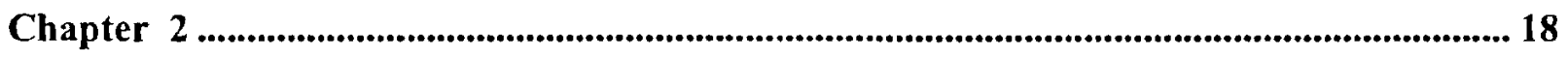

Laser Ablation Mechanism.................................................................................................. 18

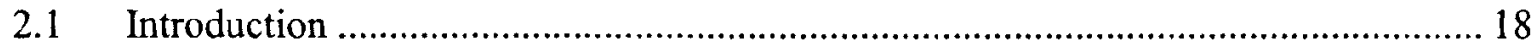

2.2 Nanosecond Laser Ablation........................................................................ 19

$2.3 \quad$ Femtosecond Laser Ablation ............................................................................ 21

2.4 Mechanisms in Laser Ablation Process .............................................................. 23

2.5 Common Optical Path Configuration ............................................................. 25

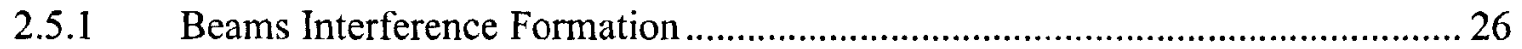

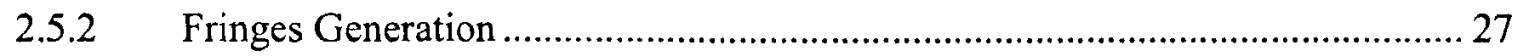




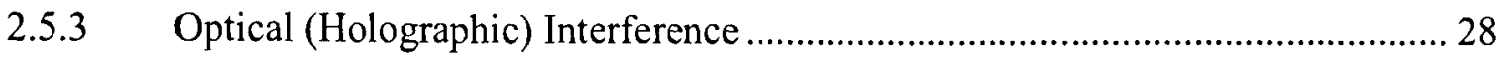

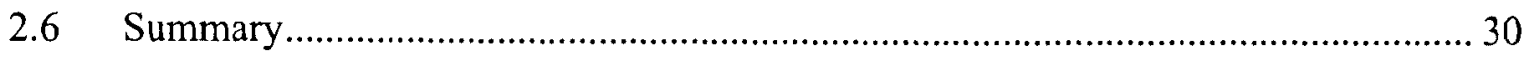

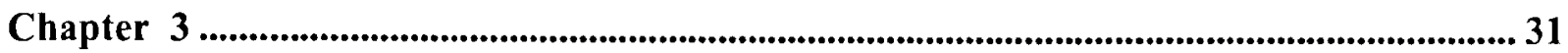

Common Optical Path Configuration for Fringes Generation ......................................... 31

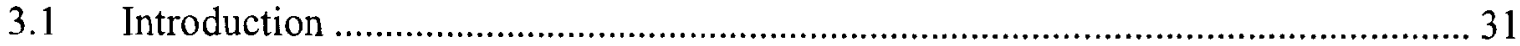

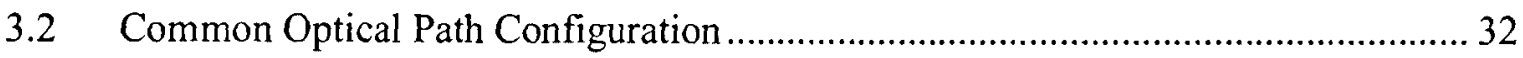

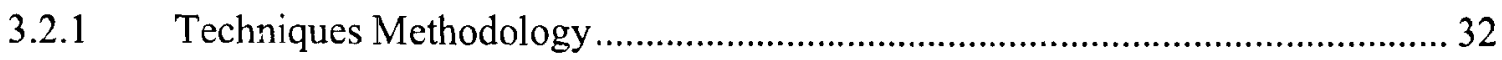

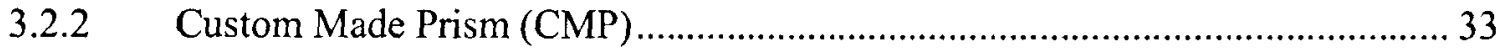

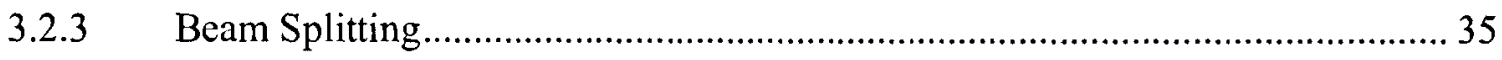

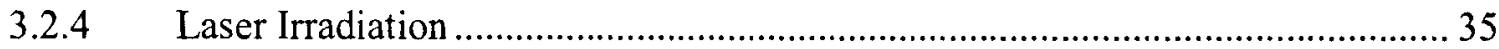

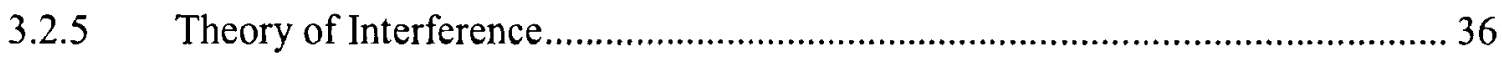

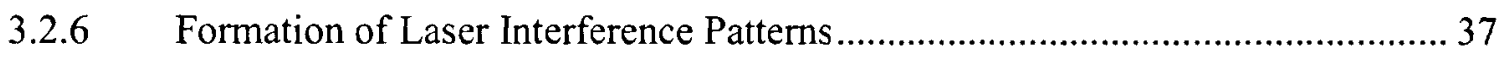

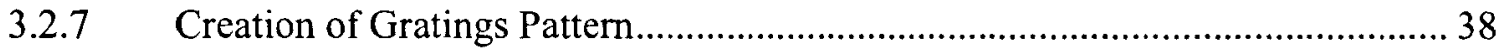

$3.3 \quad$ Summary

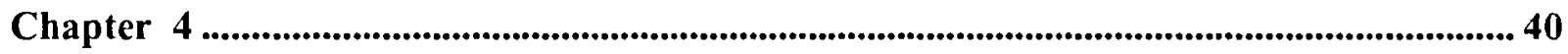

Experimental details............................................................................................................ 40

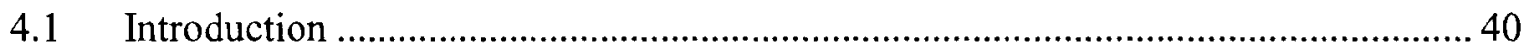

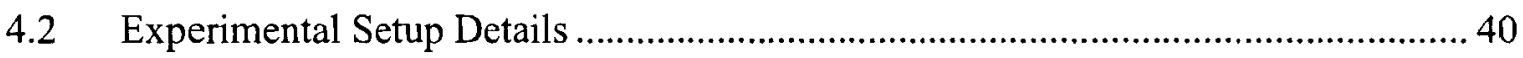

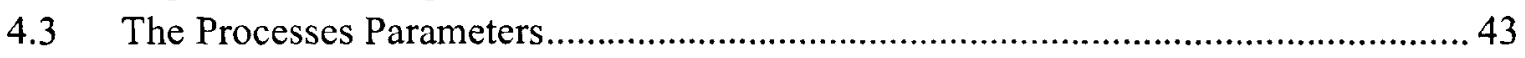

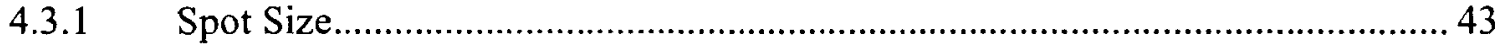

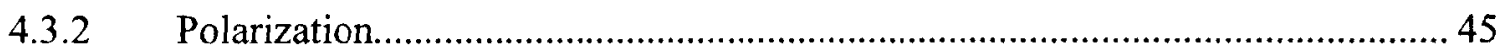

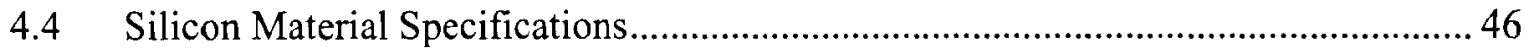

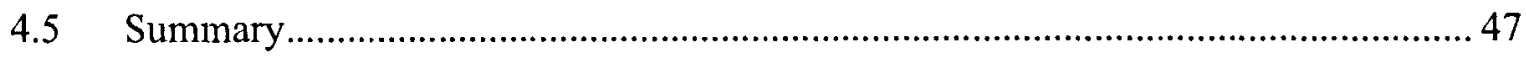

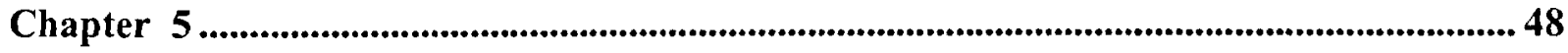

Surface Patterning of Silicon Substrate and Metallic Thin Film Using

Interferometer for Laser Irradiation Below Ablation Threshold ....................48

5.1 Introduction to Submicron and Nano Structuring of Silicon Substrate ...................... 48

5.2 Results and Discussion .......................................................................................... 52

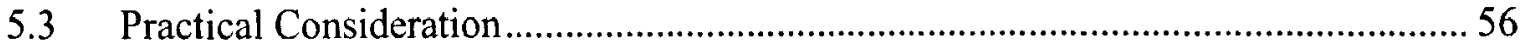

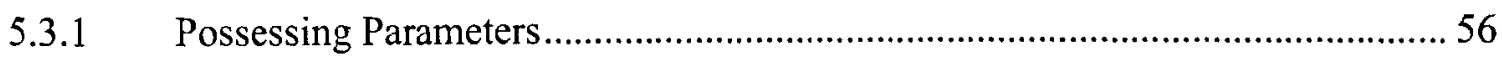

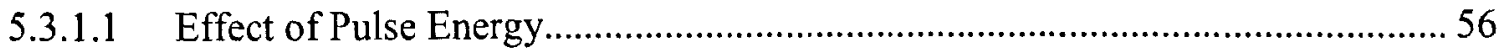

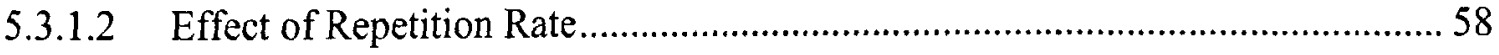




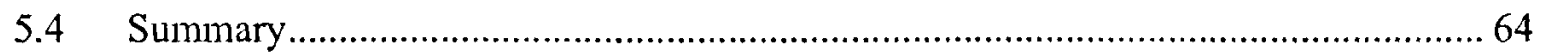

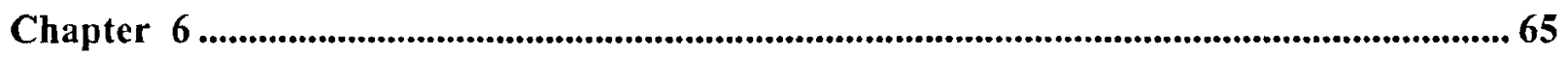

Nanosecond Laser for Silicon Nano Patterning.........................................................6 65

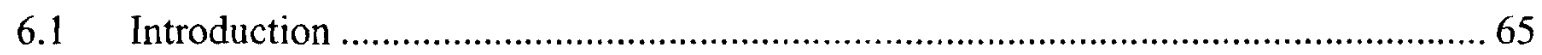

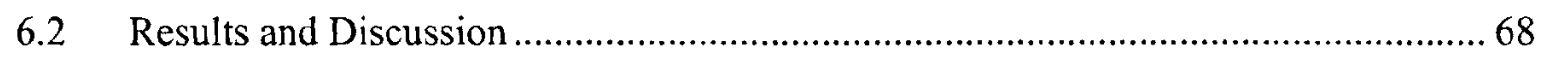

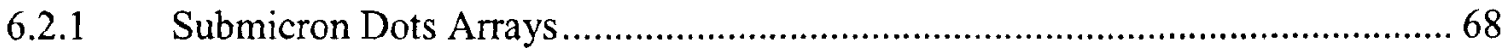

6.2.2 Ripples Patterns Induced by Interference Between Frozen Capillary Waves $(\mathrm{FCW})$ and Laser Beam ........................................................................... 73

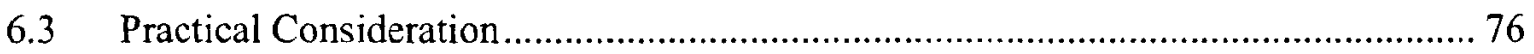

6.3.1 Prossessing Parameters ....................................................................... 76

6.3.1.1 Effect of Pulse Repetition Rate ........................................................... 76

6.3.1.2 Secondary Ripples Formation Mechanisms ............................................. 78

6.3.1.2.1 Surface Tension Waves.................................................................................. 79

6.3.1.2.2 Surface Tension Gradients ................................................................... 79

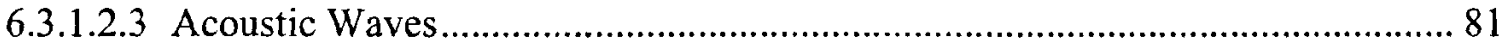

6.3.1.2.4 Interference the Incident Laser Beam With Surface Electromagnetic Wave

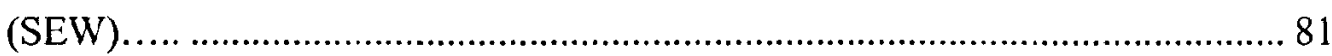

6.3.1.2.5 Interference between the Incident Laser and the Frozen Capillary Waves

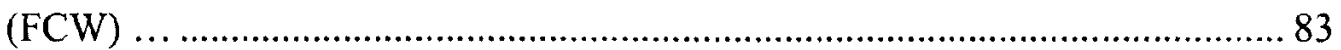

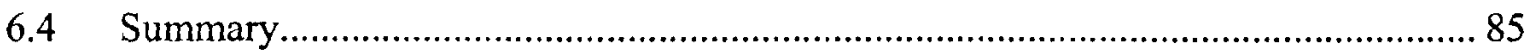

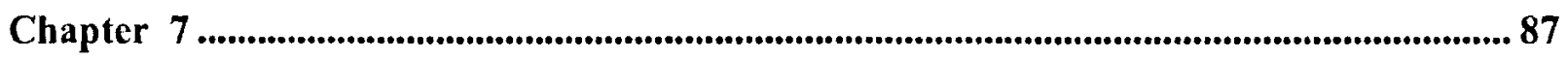

Laser Patterning Using Second Harmonic Generation Wavelength (SHG)................ 87

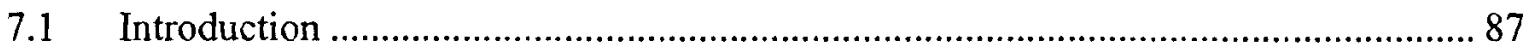

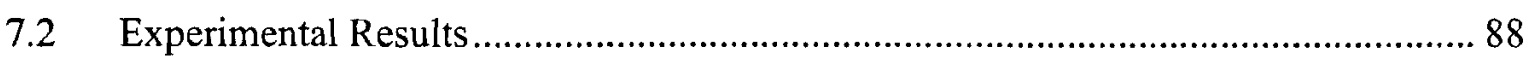

7.2.1 Gratings Induced by Long Pulses Nanosecond Laser of $532 \mathrm{~nm}$ Using Common Optical Path Interference .............................................................. 88

7.2.2 Gratings Pattern Induced by Ultrashort Femtosecond Pulses Laser of $400 \mathrm{~nm}$.. 89

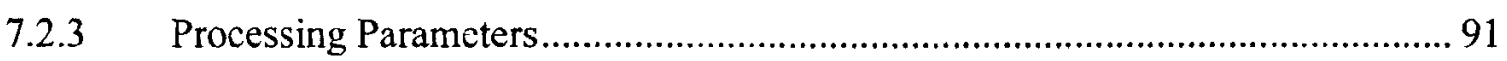

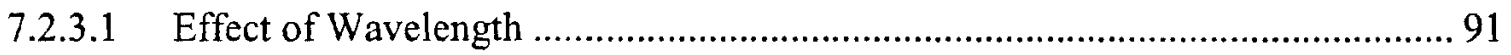




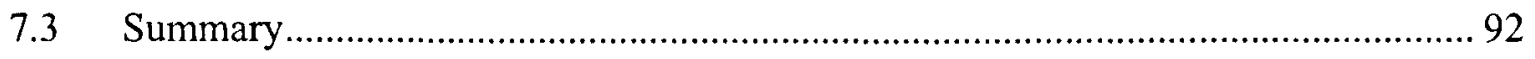

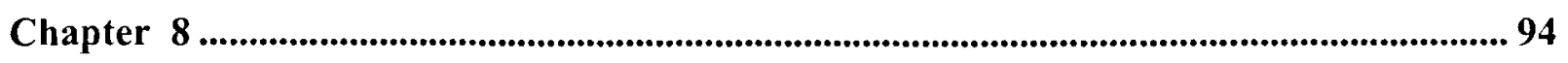

Summary, Conclusions and Future Work ....................................................................94

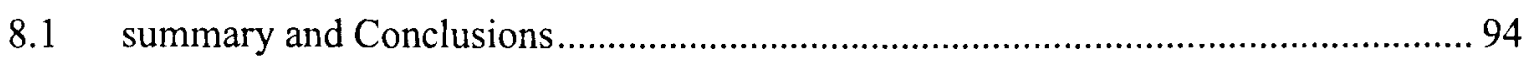

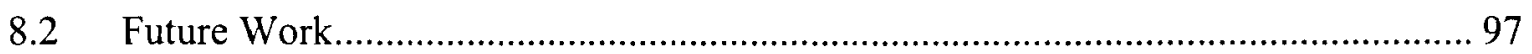

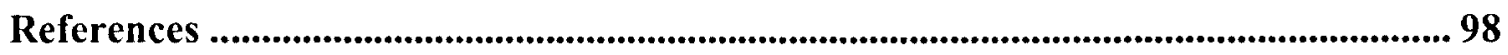




\section{List of Figures}

Figure 1-1: The two basic types of ruling tool: (a) double ended (roof edge tool) and (b) double cone tool consisting of intersecting conical surfaces. 4

Figure 1-2: Schematic representation of the mask lithography. ..................................................

Figure 1-3: Experimental setup used single laser beam fabrication. . ..........................................9

Figure 2-1: Long Pulse, Laser-Matter Interaction, displays whole view of ablation spot and it subsequent resultants.

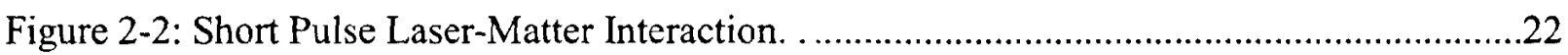

Figure 2-3: two laser beams interference pattern. ....................................................................27

Figure 2-4: Grating fabrication by optical interference of two laser beams in common optical path configuration.

Figure 3-1: The schematic diagram of common optical path configuration setup using custom-designed prism.

Figure 3-2: section $\mathrm{x}-\mathrm{x}$ of two laser beams interference that shows fringes form. .37

Figure 3-3: displays formation of interference fringes by two laser beams. 38

Figure 3-4: shows surface patterning of grating which arise by the interaction between high intensity fringes with the sample.

Figure 4-1: The schematic diagram of the experimental setup for surface patterning using common path optical configuration.

Figure 4-2: Picture of optics configuration inside the laser fabrication lab, Mechanical Engineering, Ryerson University, 2009 Toronto.

Figure 4-3: The schematic diagram of the interfering spot area shows the bonded angle between $\mathrm{x}$ - axis and single beam.

Figure 4-4: Laser spot size (do) and depth of focus $\Delta f$, for laser beam using a convex focus lens. 
Figure 4-5: Exhibits, basic definitions of $\mathrm{P}, \mathrm{S}$ and $\mathrm{C}$ polarizations.

Figure 5-1: Entire spot area for Si sample at repetition rate of $10 \mathrm{KHz}$.

Figure 5-2: Si samples at different repetition rate, (a) repetition rate of $30 \mathrm{KHz}$, pulse energy of $0.199 \mu \mathrm{J}$, (b) $20 \mathrm{KHz}$, pulse energy of $0.230 \mu \mathrm{J}$, and (c) $10 \mathrm{KHz}$, high pulse energy of $2.66 \mu \mathrm{J}$.

Figure 5-3: Si samples at different rep. rate, (a) at $40 \mathrm{KHz}$, pulse energy of $0.172 \mu \mathrm{J}$, (b) $50 \mathrm{KHz}$, pulse energy of $0.152 \mu \mathrm{J}$, (c) $60 \mathrm{KHz}$, pulse energy of $0.138 \mu \mathrm{J}$, and (d) $70 \mathrm{KHz}$, pulse energy of $0.125 \mu \mathrm{J}$. .55

Figure 5-4: At repetition rate of $10 \mathrm{KHz}$ exhibit three images of different quality at dwell time of (a) $100 \mathrm{~ms}$ (b) $150 \mathrm{~ms}$ (c) $200 \mathrm{~ms}$. 56

Figure 5-5: For different dwell time of 100,150 and $200 \mathrm{~ms}$ at repetition rate of $20 \mathrm{KHz}$ shows three results of different quality.

Figure 5-6: Threshold average beam power vs. pulse energy for low repetition rate of 10 $\mathrm{KHz}$ to $70 \mathrm{KHz}$.

Figure 5-7: Shows the grating feature quality through effect of repetition rate on line width. .58

Figure 5-8: Laser repetition rate vs, threshold average beam power. .59

Figure 5-9: SEM image of surface of sample with Au thin film after irradiation of low repetition rate of $10 \mathrm{KHz}$ and high pulse energy.

Figure 6-1: Submicron dots structure at repetition rate of $10 \mathrm{KHz}$ and pulse energy of 0.29

$\mu \mathrm{J}$.

Figure 6-2: Zoom-in of submicron dots array of multi sceneries at repetition rate of 10 $\mathrm{KHz}$ and pulse energy of $0.29 \mu \mathrm{J}$. 70

Figure 6-3: Normal view SEM images of the silicon wafer subjected to Nd: YAG laser pulses at laser fluence of $0.065 \mathrm{~J} \mathrm{~cm}-2$ : (a) Shows submicron dots array of silicon surface for partially forming zone, (b) Views of partially structuring of submicron dots at longer dwell time of $150 \mathrm{~ms}, 60 \mathrm{KHz}$, and (c) Lines 
array in consequence of dots melting at $200 \mathrm{~ms},(\lambda=1064 \mathrm{~nm}, \tau \mathrm{L}=20 \mathrm{~ns}, 10$ $\mathrm{KHz})$

Figure 6-4: SEM views of ripples at repetition rate of $30 \mathrm{KHz}$ with pulse energy of 0.199 $\mu \mathrm{J}$ .75

Figure 6-5 (a): Threshold Energy Fluence vs. Repetition Rate from $10 \mathrm{KHz}$ to $30 \mathrm{KHz}$. .77

Figure 6-5 (b): Threshold Average Beam Power vs. Repetition Rate from $10 \mathrm{KHz}$ to 30

$\mathrm{KHz}$.

Figure 7-1: shows SEM image of the grating fabricated using p-polarization of second harmonic generation of $532 \mathrm{~nm}$ of wavelength at repetition rate of $50 \mathrm{KHz}$ and pulse duration of $20 \mathrm{~ns}$.

Figure 7-2: shows AFM image of the grating formed at polarization parallel to the propagation plan employed second harmonic generation wavelength of short pulse femtosecond laser of $400 \mathrm{~nm}$ and short pulse duration of $150 \mathrm{fs}$

Figure 7-3: The grating period is measured as about $220 \mathrm{~nm}$ that induced by femtosecond laser of 400 ns wavelength 


\section{List of Tables}

Table 4-1: C-Silicon specifications.

Table 5-1: The calculation of laser intensity at different repetition rates. ...............................52

Table 5-2: Thermo-physical and optical properties of Au. ...........................................64

Table 6-1: Silicon parameter used to solve the Equations 5-2 and 5-3. ..............................78 


\section{Nomenclature}

$\Lambda \quad$ Capillary wave spacing (period), Ripple spatial period, Dot array spacing surface tension coefficient of molten silicon

$p$ mass density of liquid layer

$\lambda, \lambda_{0}$ wavelength of the laser beam

$\tau_{\mathrm{L}} \quad$ pulsewidth

$\tau_{L} \quad$ life time of the molten state

$\tau_{\mathrm{e}} \quad$ electron cooling time

$\tau, \tau_{P} \quad$ pulse duration, pulsewidth

$\tau_{i} \quad$ lattice heating time

$\delta \quad$ skin depth

E energy deposited inside target per unit mass

$\Omega \quad$ specific heat of evaporation

$\alpha \quad$ surface transmissivity

$\gamma \quad$ parameter characterizing electron-lattice coupling

$\Delta d \quad$ path difference

$\Delta \lambda \quad$ bandwidth

$\Delta \theta \quad$ divergence angle

$\theta \quad$ interference angle (incident angle)

$\emptyset_{\text {th }} \quad$ laser threshold ablation

$\triangle \mathrm{f}, \mathrm{DOF} \quad$ depth of focus

$\sigma$

surface tension coefficient

$\sigma_{0}$

surface enthalpy

$\mathrm{n}$ response function

$\tau_{\mathrm{h}} \quad$ integer multiple of $\Sigma_{\mathrm{s}}$

$\mu \mathrm{J} \quad$ micro Joule $\left(10^{-6} \mathrm{~J}\right)$

$\mu \mathrm{m} \quad$ micrometer $\left(10^{-6} \mathrm{~m}\right)$

$\gamma \quad$ surface tension

$\omega \quad$ angular frequency of incident light 
a

A

A

$\AA$

$\mathrm{Ag}$

As

AFM

$\mathrm{C}$

$\mathrm{C}_{\mathrm{e}}$

$\mathrm{C}_{\mathrm{i}}$

$\mathrm{CO} 2$

CMP

$\mathrm{Cr}$

$\mathrm{Cu}$

$\mathrm{C}-\mathrm{Si}$

$\mathrm{CW}$

$\mathrm{C}_{\mathrm{v}}$

d

dd

$\mathrm{d}_{\mathrm{o}}$

D

$\mathrm{D}_{\mathrm{e}} \quad$ electron thermal diffusivity

DRP direct writing process

$\mathrm{d} \sigma / \mathrm{dT} \quad$ temperature coefficient

E electrical vector

$E_{p} \quad$ laser pulse energy

$\mathrm{eV} \quad$ electron volt (unit of energy)

F laser fluence

$F, f \quad$ effective focal length of focused lens

$F_{t h}, \varnothing^{\text {th }}, \dot{\varnothing} \quad$ threshold fluence

$\mathrm{F}_{\mathrm{a}} \quad$ absorbed laser fluence, ablation fluence

Fs, fs femtosecond $\left(10^{-15} \mathrm{~s}\right)$

FCW frozen capillary waves 


$\begin{array}{ll}\mathrm{g} & \text { acceleration field } \\ \mathrm{Ga} & \text { gallium } \\ \mathrm{GW} & \text { gigawatt }\left(10^{9} \mathrm{~W}\right) \\ \mathrm{h} & \text { height/depth of the molten layer } \\ \mathrm{HAZ} & \text { heat affected zone } \\ \mathrm{HD} & \text { heat diffusion coefficient } \\ \mathrm{Hg} & \text { Mercury } \\ \mathrm{Hz} & \text { hertz } \\ \mathrm{I}_{0,} \mathrm{I}_{\mathrm{a}, \mathrm{I}} & \text { laser intensity } \\ \mathrm{I}_{\mathrm{th}} & \text { threshold intensity } \\ \mathrm{I}(\mathrm{t}) & \text { laser intensity at time } \mathrm{t} \\ \mathrm{k} & \text { wave number, wave vectors } \\ \mathrm{k}_{\mathrm{L}} & \text { wave vector of the incident radiation } \\ \mathrm{k}_{\mathrm{e}} & \text { electron thermal conductivity } \\ \mathrm{k}_{\mathrm{o}} & \text { conventional equilibrium thermal conductivity } \\ \mathrm{k}_{\mathrm{s}} & \text { real part of the propagation wave vector } \\ \mathrm{KHz} & \text { Kilohertz }\left(10^{3} \mathrm{~Hz}\right) \\ \mathrm{L}_{\mathrm{m}} & \text { specific heat of melting } \\ \mathrm{LIPSS} & \text { laser induced periodic surface structures } \\ \mathrm{Mo} & \text { Molybdenum } \\ \mathrm{ms} & \text { microsecond } \\ \mathrm{MW} & \text { megawatt }\left(10^{6} \mathrm{~W}\right) \\ \mathrm{Nd}: \mathrm{YAG} & \text { neodymium: } \text { yttrium-aluminum-garnet } \\ \mathrm{nm} & \text { nanometer }\left(10^{-9} \mathrm{~m}\right) \\ \mathrm{Ns}, \mathrm{ns} & \text { nanosecond }\left(10^{-9} \mathrm{~s}\right) \\ \mathrm{N} & \text { number of grating lines } \\ \mathrm{NA} & \text { numerical aperture } \\ \mathrm{P} & \text { power } \\ \mathrm{Ps}, \mathrm{ps} & \text { picosecond }\left(10^{-12} \mathrm{~s}\right) \\ \mathrm{PMMA} & \text { poly-methyl methacrylate } \\ \mathrm{Q} & \text { heat flux } \\ \mathrm{R} & \text { reflectivity } \\ \mathrm{Re} & \text { Rhenium } \\ \end{array}$




$\begin{array}{ll}\mathrm{R}_{\mathrm{r}} & \text { repetition rate } \\ \mathrm{S} & \text { laser heating source term } \\ s & \text { surface entropy } \\ \mathrm{SEM} & \text { scanning electron microscopy } \\ \mathrm{SEW} & \text { surface electromagnetic waves } \\ \mathrm{SiO} 2 & \text { silicon dioxide } \\ \mathrm{t} & \text { time } \\ \mathrm{T} & \text { temperature } \\ \mathrm{T}_{\mathrm{e}} & \text { electron temperature } \\ \mathrm{T}_{\mathrm{i}} & \text { lattice temperature, initial temperature } \\ \mathrm{T}_{0} & \text { temperature at time }=0 \\ \mathrm{~T}_{\mathrm{m}} & \text { melting temperature } \\ \mathrm{UV} & \text { ultra violet } \\ \mathrm{V} & \text { beam velocity vector } \\ \mathrm{V}_{\mathrm{C}} & \text { critical velocity of the Jaser beam } \\ W & \text { watt } \\ W & \text { ripple base width } \\ \mathrm{z} & \text { direction perpendicular to target surface }\end{array}$




\section{CHAPTER 1 \\ INTRODUCTION}

\subsection{INTRODUCTION TO SUBMICRON AND NANO SURFACE PATTERNING TECHNIQUES}

Fabrication of surface patterns structures in micro and nanometer scales of various materials is one of the most important applications in micromachining domain. According to [1], microtechnology has changed our lives immensely. Concurrently, the demand for more precise and more efficient apparatuses is driving the semiconductor industry towards the use of different materials of thin films fabricated by pulsed laser. It has attracting interest in increasing the densities of integrated circuits, quantum devices, and recording media. Also in optoelectronics and optical telecommunications as well as IC chips technology which are the most today's essential industries to sustain our lifestyles. Such as these versatile patterns have great interest for fundamental techniques in the development of semiconductor applications. However, the fabrications of such surface patterns like grating and dot array have been a major challenge. For instance, nanodot fabrication techniques have been suggested to meet the severe requirements of generate highly efficient dots arrays by disposing dot density, dot shape, dot pitch and array spacing.

As a summary, fabrication methods at micro and nanometer scale are developing more important from the industrial applications viewpoint. These technologies have uscd to fabricate surface patterns at micro and nano scales on various materials. Nanosecond and femtosecond 
pulsed laser ablation therefore is preferred techniques for precise patterning over the large-scale machining area and it can be considered a capable performance.

In the following sections, the principal techniques for micro and nanofabrication pattern formation are described and represent by these techniques. The applicability of each for a particular purpose is determined both by the intrinsic optical characteristics of the pattern forming technique and by the characteristics of the specific machining process. The emergent laser patterning techniques is touched upon and will be described totally in Chapter 1, Sections

\subsection{2-1.1.6}

\subsubsection{MECHANICAL GROOVES SCRIBING}

Conceptually, a grating is a simple pattern constituting periodic lines and spaces. Before the development of the laser holography recording techniques, all the optical gratings were made by the method of mechanical ruling. The grating ruling work needs high precision machine and is very time consuming and can be performed only in a few shops. As referenced from [2] a ruled grating is produced by a ruling engine that cuts grooves into the coating on the grating substrate (typically glass coated with a thin reflective layer). This classical method of manufacturing gratings, used for over 150 years, is to scribe, burnish or emboss a series of grooves upon a good optical surface. Originally this surface was one of highly polished speculum metal but ever since vacuum coating techniques were developed in the 1930s the majority of gratings have been ruled in thin layers of aluminum or gold deposited upon a glass substrate. 
Basically, there are two problems in ruling gratings, the first is to produce consistently a groove of the right shape and the second is to ensure that it is put in the right place. The profile of the groove is important, but in principle it is generally less crucial than that of maintaining a high precision in positioning. The shape of the groove determines the efficiency of the gratings and its quality (degree of surface roughness and jagged edges) will influence the level of diffuse scattering. If there are variations in depth or shape along the length of the groove, these can generate stray light. The density of the grooves determines the dispersion and the accuracy of position of the grooves determines the quality of the spectral image. The tool is usually polished to an angle of $90^{\circ}$ or more in order to give the required groove shape. In order to attain maximum hardness it is necessary carefully to align the tool with respect to the crystallographic axes. There are two common forms of ruling diamonds as illustrated in Figure 1-1. The first is a straight chisel or roof shape and the groove is formed by dragging the edge of the tool through the metal. This type is comparatively easy to align and since only a small area is in contact with the metal it requires a comparatively light loading. However, the stresses on it are concentrated upon a smaller area and when it does fracture or wear, it must be discarded. The second type is boat shaped and the ridge of the tool is a curve defined by the intersection of two cones. This has the advantage that different sections of the tool may be used for different gratings. Therefore tool life is extended. But that does require far greater loads and more precise alignment than with the roof type. 


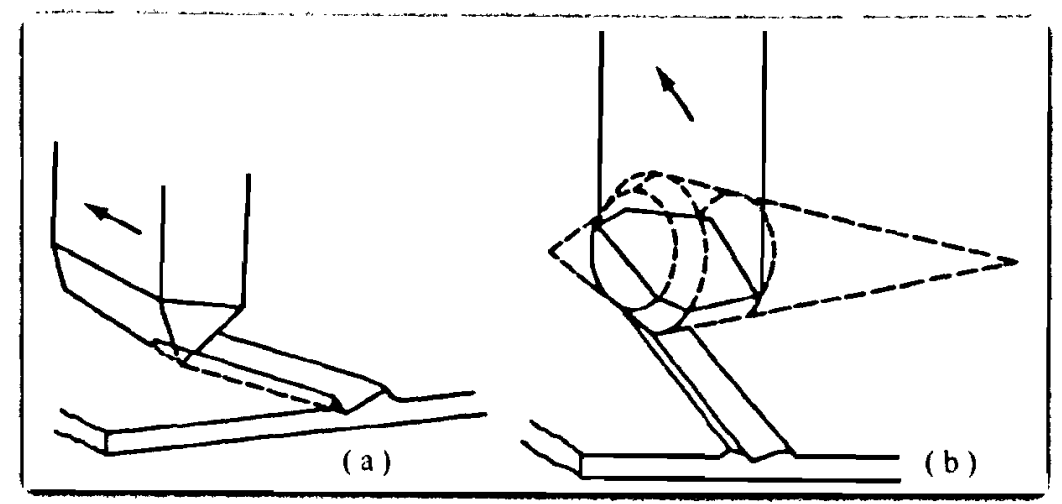

Figure 1-1: The two basic types of ruling tool: (a) double ended (roof edge tool) and (b) double cone tool consisting of intersecting conical surfaces [2].

When a series of grooves is ruled, the shape of a given groove is changed as the subsequent groove is ruled. Material will be pushed away from the tool and this will tend to steepen the angle of the adjacent facet. It is therefore preferable to arrange that each new groove is ruled on the side of the steep facet of the existing grooves. Practically all modern ruled gratings are done in a layer of metal, usually aluminum or gold, which has been vacuum deposited upon the surface of an optically flat glass substrate. The most notable exception is gratings used with high powered infrared lasers, in which case the grooves are ruled directly onto a substrate of copper that has been designed for the rapid extraction of heat.

The quality of the gratings depends not so much upon the optical properties of the film but upon its mechanical properties and the tolerances on imperfections is far for more severe. The problem which has received by far the most attention is to maintain high position precision. The tolerances involved are probably the most severe of any mechanical operation and in some cases demand accuracies of the order of atomic dimensions. The ruling tool travels large distance to rule one grating; also the ruled grating is formed by burnishing each groove individually. It may take several weeks to rule a long grating. For example, a square grating of dimensions $100 \times$ 
$100 \mathrm{~mm}$ with 1000 grooves per millimeter will require the diamond to move $10 \mathrm{~km}$ (over six miles).

In the next section the non-conventional (Lithographic / Holographic) laser patterning techniques will be fully described, such as Phase Mask, Direct Writing Process, Holographic Interference of two laser beams, and other techniques.

\subsubsection{CONTACT / PROXIMITY MASKING FABRICATION TECHNIQUE}

Proximity masking may be used for patterning photoresists in a conventional photolithographic process. In this technique, the mask is either separated from substrate by only a very small distance or is in direct contact with the substrate. The ratio of the feature size of the mask and the actual feature size of the replica is approximately 1:1. No focusing optics is required and uniform illumination across the substrate is desired. The pattern will form by exposing followed by developing, typically with a wet etching solution, or by a direct of one step photochemical reaction. The optical resolution of this approximation is given by Fresnel diffraction,

$$
\omega^{2}=\frac{\lambda z}{2}
$$

Where $\lambda$ is the light wavelength, $z$ is the mask to surface separation and $\omega$ is angular wave frequency. For a small feature sizes, diffraction takes place. Light guiding effects decrease the effective resolution as holes are machined deeper undercutting can occur by scattering light on to the sidewalls. This effect does not depend on the laser light intensity but on the machining depth and the diffraction orders. The nonzero intensity of laser light in the shadow of the mask 
can cause undercut; higher laser light intensity can make this problem more serious. Further increases in laser intensity have slightly effect on the etching rate for bottom of the hole but continue to increase the rate of sidewall erosion. Therefore, higher intensity light will be damaging to the process resolution.

Furthermore proximity masking for ablation, machining of polymers lets some degree of edge profile control. Adjustment of the mask to substrate separation makes it possible to vary this edge profile from nearly vertical to highly taper. This tapered profile at the mask edge determines the machining depth per pulse through the strong fluence dependence typical of polymer ablation. Exposing Light for a longer time than required leads to increased tapering from the sidewall. The minimum number of laser pulses necessitated to remove all polymers from a substrate is determined by the fluence at the Fresnel diffraction. Moreover, the thickness of the polymer layer also affects the etch profile since more laser pulses are required to totally ablate of a thicker layer. The Longer wavelength laser can give a somewhat more vertical sidewall than does shorter wavelength laser. Notwithstanding the wider lateral spread produced at the pattern edge by the longer wavelength, the higher threshold fluence required for polyimide ablation at $388 \mathrm{~nm}$ vs. $248 \mathrm{~nm}$ more than compensates for the wider spread. High resolution machining has been achieved with contact or proximity masking. Exposure of a methylmethacrylate methacrylic acid copolymer with $157 \mathrm{~nm}$ laser followed by wet development has produced $0.13 \mu \mathrm{m}$ lines. Single step fabrication of $0.3 \mu \mathrm{m}$ grooves in Mo has been achieved using interference fringes from a single narrow slit proximity mask with laser wavelength of 193 $\mathrm{nm}[4]$. 


\subsubsection{PHOTOLITHOGRAPHIC PATTERNING}

Photolithographic techniques which use image projection and proximity or contact masking are employed in conventional patterning processes. In the image projection technique, an image of a mask placed remotely from the substrate surface is demagnetized by projection optics to give the requested pattern at the surface as shown in Figure 1-2. Commercially available exposure tools that operate in the short wavelength laser visible and near ultra violet usually uses refractive optics. However, reflecting optics instead of refracting optics are highly beneficial for shorter wavelengths since they enable the use of ultraviolet (UV) laser without the problem of laser absorption (a) in the optical elements and the difficulty of chromatic correction. The Rayleigh criterion for diffraction limited feature size for image projection is given by,

$$
\omega=\frac{1.22 \lambda}{2(\mathrm{NA})}
$$

Where $\lambda$ is the wavelength in the medium in contact with the substrate and NA is the numerical aperture $(\mathrm{NA}=$ optical element diameter/ $(2 \times$ focal length $))$. A large numerical aperture improves transmission of the higher spatial frequencies. Submicrometer features scale is easily achieved using image projection techniques. For example, gratings with $0.2 \mu \mathrm{m}$ spacing can produce on Si using wavelength laser of $193 \mathrm{~nm}$. However, power densities in excess of 2 MW. $\mathrm{cm}^{-2}$ have produced a loss of resolution when etching organic materials $[4,5]$. 


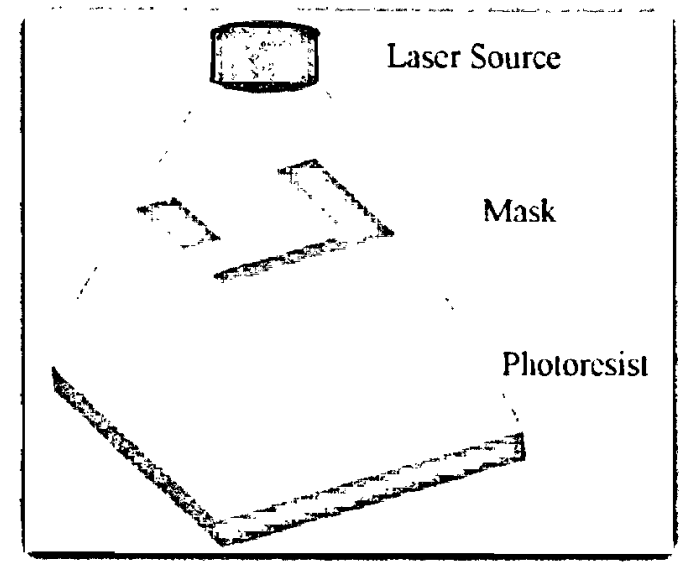

Figure 1-2: Schematic representation of the mask Photolithography.

\subsubsection{DIRECT WRITE PROCESSING (DRP)}

The direct writing technique does not employ a mask to define the pattern. Instead the output beam of a laser is focused directly onto the substrate surface. The beam may be held stationary to fabricate a hole in the substrate or scanned to produce a grating pattern. There are one step and two steps of direct write processes. In a one step process material is removed concurrently with the irradiation beam. While in the two step process a resist is exposed by the laser but its removal is effected during a subsequent development step similarly in conventional photolithography. An interference pattern can be generated from a single collimated monochromatic coherent laser beam if it is made to reflect back upon itself, as illustrated in Figure 1-3. 


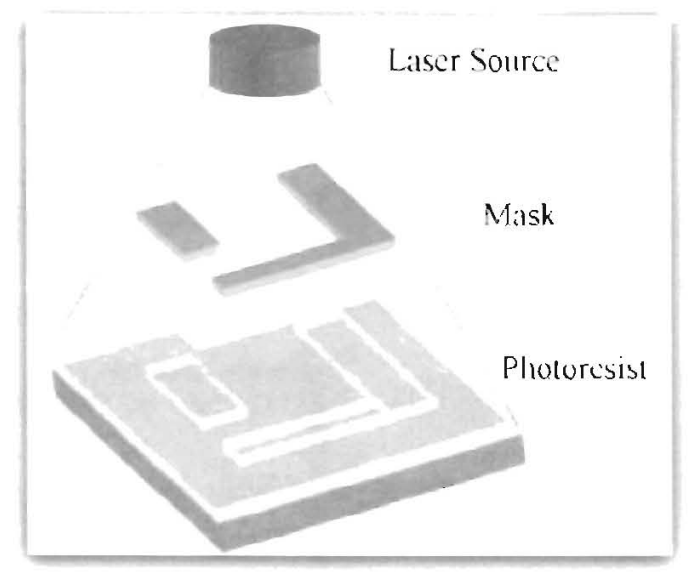

Figure 1-2: Schematic representation of the mask Photolithography.

\subsubsection{DIRECT WRITE PROCESSING (DRP)}

The direct writing technique does not employ a mask to define the pattern. Instead the output beam of a laser is focused directly onto the substrate surface. The beam may be held stationary to fabricute a hole in the substrate or scanned to produce a grating pattern. There are one step and two steps of direct write processes. In a onc step process material is removed concurrently with the iriadiation beam. While in the two step process a resist is exposed by the laser but its removal is effected during a subsequent development step similarly in conventional photolithography. An interference pattern can be generuted from a single collimated monochromatic coherent laser beam if it is made to reflect back upon itself. as illustrated in Figure 1-i. 


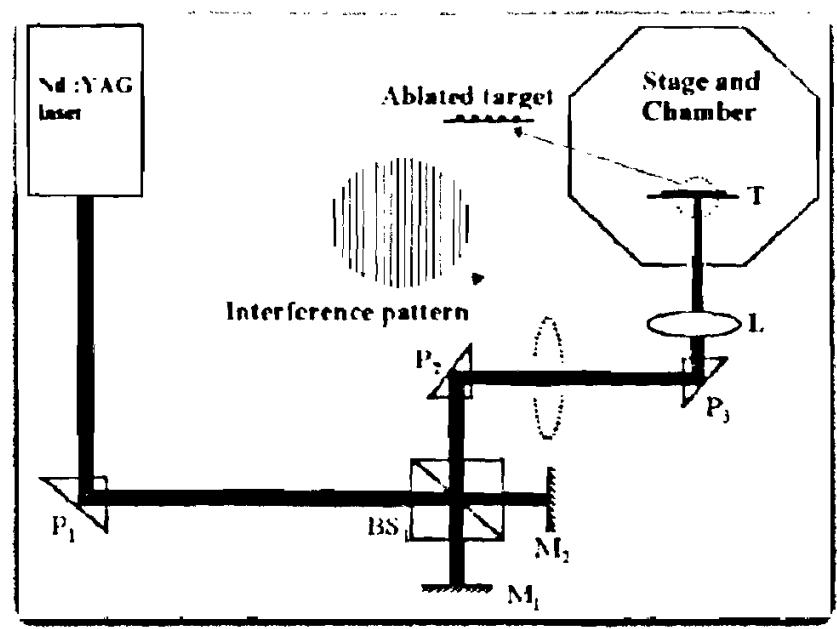

Figure 1-3: Experimental setup used single Iaser beam fabrication [3]

In Figure 1-3, a Michelson interferometer was assembled with high damage threshold beam splitter BS1 and two mirrors M1 and M2. The interferometer was illuminated with second harmonic of high power Q-switched Nd: YAG laser with the help of a 90 prism P1. The output of this interferometer consists of straight parallel fringes. Periodicity of this pattern depends on the wavelength and the angular separation between two interfering beams, which can be controlled by adjusting the tilt of one of the mirror online. The interference pattern steered with help of two 90 prisms, P2 and P3, as shown, on stage into chamber. The laser beams are traveling at certain "very small" angular separations "few mrad". Using wavelength value of $532 \mathrm{~nm}, 593 \mathrm{~nm}$ grating spacing have been achieved [3].

A standing wave pattern will be formed, with intensity maxima forming planes parallel to the wavefronts. The intersection of this interference pattern if a photoresist covered substrate will yield on its surface a pattern of grooves, again whose spacing, $d$, depends on the angle between incident light and the substrate surface, $\theta$. The definition of an incident angle, $\theta$, is illustrated in Figure 3-3. The relation between, $d$, and, $\theta$, is given by Equation 1-3, 


$$
\mathrm{d}=\frac{\lambda}{2 \sin (\theta / 2)}
$$

It must be emphasized that the recording geometry behind the single beam holographic grating is different from that of the interferometer. The arrangement of focusing optics decides whether or not the laser spot size at the surface is diffraction limited. A diffraction limited spot size can be achieved only by placing the final optical element very close to the substrate surface. Moreover high image precision requires a very flat surface because of the very shallow depth of field. When longer working distances are used, a larger minimum spot size, which is determined by the focused laser beam waist, results but the depth of field is increased. For a Gaussian profile beam, the diffraction limited spot size at $1 / \mathrm{e}^{2}$ of the maximum intensity is given by,

$$
\omega=\frac{\lambda}{\mathrm{n}(\mathrm{NA})}
$$

Where $\lambda$ is vacuum wavelength and $n$ is the index of refraction of the medium in contact with the substrate surface. Submersion of the last optical element in a solution with a high index of refraction gives a smaller spot size. The resolution of direct write processes is often influenced by thermal process effects, since many direct write processes employ ordinary chemical reactions that are thermally activated. Features can be produced which are either larger or smaller than the original beam diameter (D), depending on the total energy deposited, the thermal diffusion properties of the substrate and the energy deposition rate. In general, narrower features are produced by pulsing or rapidly scanning the beam, while wider features are produced if the beam is stationary or scanned slowly. This is a consequence of the exponential dependence on temperature exhibited by thermally activated chemical reactions and the dependence of the substrate temperature profile on the rate of laser energy deposition. Other 
parameters influences the ablation process in pulsed lasers are the pulse shape, pulse duration and energy density which affect the temperature rise and the thermal gradient profile. Resolution can be degraded when an unsuitable combination of laser power and scan speed produces ripple like structures. The comparative resolution of image projection and one-step and two-step direct writing depends on particular process characteristics. One step direct writing using a thermally activated chemical process with a fairly large activation energy, and therefore a large contrast parameter, can produce a minimum line spacing which is one fourth that possible using image projection. A two step direct writing process has a resolution between one step direct writing and image projection technique depending on its nonreciprocity parameter $[1,4]$.

\subsubsection{PHOTOCHEMICAL FORMATION}

In this method the substrate itself is altered in some patterned way prior to exposure to uniform, unpatterned laser intensity. This approach is especially convenient for carrier controlled photochemical etching, where photo generated electrons and/or holes drive the etching chemistry. Under such conditions alteration of the carrier concentrations by altering substrate electronic properties such as recombination results in substantially different photochemical machining rates in the altered and unaltered regions of the surface. Two examples of this involve drastically increasing the carrier recombination rate by either ion implantation or impurity introduction. Ion implantation has been used to produce recombination promoting damage in semiconductors to suppress both wet photoelectrochemical etching and dry photochemical etching of GaAs using a Hg lamp as the photon source [5]. 


\subsubsection{PATTERNING USE FEMTOSECOND LASER}

The main advantage of using femtosecond laser pulses for the ablation of materials comparing with using nanosecond laser pulses, the heat diffusion into the target is negligible, which results in highly localized ablation and precise patterning of the sample without much thermal damage to the surroundings $[7,8]$. Current reasoning suggests that the pulsewidth of a femtosecond laser is too short to allow for heat conduction into the bulk of the ablated material. Therefore, defects associated with thermal heating during ablation can be eliminated and superior machining quality can be obtained. With new techniques in fiber amplification, high power femtosecond lasers are now available on the market. There are valuable researches carried using femtosecond lasers as attractive alternative for surface patterning in machining of a variety of materials has created huge interest [43-47]. Therefore, using a femtosecond laser for surface patterning applications involving a variety of materials could be considered a feasible and promising industrial tool. This method will be explained in detail in Chapter 7, Sections 7-2-2 and 7-2-3 of the thesis.

\subsection{SUMMARY OF SUBMICRON AND NANO STRUCTURING TECHNIQUES}

Besides the mechanical grooving scribing technique which shapes a ruled grating by burnishing each groove individually; the ruling diamond may travel a very large distance to rule one grating. The quality of the gratings depends upon its mechanical properties, (optical quality and particularly the surface smoothness, is often improved by ruling) and the tolerances on imperfections are far for more severe. The problem which has received by far the most attention is the positioning accuracy. So, the disadvantage of mechanical scribing that grating ruling work needs high precision machine is very time consuming and can be performed only in a few shops. 
While in such other conventional techniques, there are some parameters such as wavelength, laser intensity, pulse duration and its coherence that influence pattern resolution and quality. The most fundamental limiting factor is the wavelength since, clearly, the shortest wavelengths permit the highest optical resolution. However, other process considerations, such as absorption by reactant precursors, substrate material selectivity based on band gap, or minimization of substrate damage from (UV) photons [4], may make it more advantageous to use longer wavelengths despite the decrease in optical resolution. Depending on the pattern formation technique, coherence might be either essential, as in holographic etching, or undesirable, as in projection etching and proximity masking. Some laser sources, such as Nd: YAG has long coherence lengths. At the same time, the ablation process depends on thermal and optical properties of the materials. In consequence, such these methods can be used to produce useful patterns, like gratings, on the surface of a substrate by periodically varying the intensity of light at the surface using the interference technique. There are a number of possible pattern formation techniques that can be employed in typical conventional laser fabrication. The achievable resolution for a particular technique is often determined both by the inherent optical resolution and by specific process characteristics. The laser source for these typical conventional techniques produces pulses with the duration in the range of nanoseconds to milliseconds. Besides the advantages of fabricating with laser, it has several limitations. The thermal load in the material due to the heat conduction is relatively high which results in large (HAZ) that may damage the delicate circuitry in the vicinity. Moreover a large amount of melting also affects the quality of finishing. As a conclusion for this Chapter, in order to meet the future demand for fabricating patterns of features size below $100 \mathrm{~nm}$ can be possible with nanosecond laser of $2^{\text {nd }}$ harmonic generation and up. Nanosecond laser fabrications apply easy setup with minimum optical parts, 
although it performs large (HAZ). The next step it can be transferring the same setup for patterns fabrication using femtosecond laser which perform less (HAZ), and can be considered as practical solution with laser fabrication.

\subsection{RESEARCH OBJECTIVES}

The use of nanosecond laser source still has unsatisfactory experimental studies in the field of laser fabrication. The main goal of this thesis is to study the low power and below threshold repetition rate "surface patterning by laser interference in common optical path configuration" using nanosecond laser system and its effect on the pattern quality and specifications. The advantage of using femtosecond laser systems for novel laser processing techniques in nano and micro scale has a wide application in micromachining area. At the same time the capabilities of the laser systems were using at the earlier time did not have the potential of overcoming the throughput and grating spacing requirement. The feasibility of using nanosecond lasers system in grating fabrication is un-complex setup and procedures which greatly depends upon the laser power and repetition rate. The primary difficulty of direct fabrication grating by femtosecond interferometric technique is to align two femtosecond pulses travel exactly the same optical path. Eventually, scrutinize the morphology of the pattern spacing that controlling by several experimental conditions, mainly by exposure time. The very short dwell time will prevent thermo stress, fatigue and material hardness with the minimum of structure modifications then increase the sample specification. Therefore, the outline of the research objectives of this thesis can be summarized as,

1. Develop a self-aligned optical configuration for direct grating writing.

2. Study the influence of laser parameters on the grating writing. 
3. Develop a method for the generation of nanodots arrays.

4. Investigate phenomenon associated with grating writing.

\subsection{OVERVIEW OF THESIS}

In Chapter 2, the pulsed laser ablation mechanism is discussed in general. Existing mechanism and theories describing laser matter interaction during the long pulse and short pulse ablation of silicon are summarized, with exceptional consideration to nanosecond laser ablation. Direct grating writing for holographic interference fabrication technique in common optical path was demonstrated as well as the fringes generation. In Chapter 3, common optical path configuration for fringes generation in details was displayed. The explanations discussed the existing custom made prism, beam splitting, theory of interference, formation of interference pattern, and creation of grating pattern. Chapter 4 demonstrates the research experimental setup in details, also important laser parameters are discussed and substantiated the role of influential parameters. Chapter 5 bestowed a methodical experimental study in addition investigated the influence of pulse energy, low repetition rate of $10-70 \mathrm{KHz}$ using low power nanosecond laser on grating patterns which achieved a shorter period (d) of $1300 \mathrm{~nm}$ by applying a fundamental wavelength of $1064 \mathrm{~nm}$. Discussed and verified the morphology of the modified silicon pattern, prevented the thermo stress, fatigue and material hardness with the minimum of structure modifications in order to increase the sample specification. Decisive parameters such as the ablation threshold and repetition rate are inferred as a function of grating period width, and the grating shape, which is distinguishable and acuter at higher repetition rates. The threshold energy fluence (threshold ablation) is found to decrease and the threshold beam power increases slightly with the increase in the pulse repetition rate. The results obtained have been donated to the 
experimental analysis of the effect of pulse repetition rate on the ablation threshold and fabricating pattern quality utilizing nanosecond lasers with low threshold power. Beneficial indicator for line width shape variation was seen as a function of laser repetition rate. As shown in Figure 5-7 the line width is inverses proportional with the influence of repetition rate. The molten debris and its dependence upon the longer dwell time of $200 \mathrm{~ms}$ totally provide unclear pattern that demolish the grating shape have been found. The quality of the pattern obtained and the amount of debris seen decrease at a shorter time and higher machining speed.

A systematic study of the influence of the fixed pulse width of $20 \mathrm{~ns}$, repetition rate, pulse energy, and the machining time on the dots array formation, the dots separation and its spacing that created during the surface patterning operations, as well as the formation mechanism of secondary patterning of ripples in submicron scale are studied in Chapter 6. The formation of regulated submicron dots arrays and the dynamics of the interaction between a single shot of two interfered nanosecond laser pulses and Silicon wafer are discussed. The dots arrays was governed and arose from intersect two patterns, first is grating that generated by direct interference writing formula and second is ripple that generated by interference between the incident laser and the frozen capillary waves (FCW). A number of different experiments were performed that compares the influence of laser parameter on the profile and quality of fabricating sub-micron dots patterns. The experimental results attained by using the low power, low repetition rate nanosecond laser are encouraging for surface patterning of silicon wafers. The mechanisms and theories guide to the formation of dots and ripples in both the nanosecond and femtosecond pulse width ranges were explained. The influence of the machining time, pulse energy and laser parameters on the machining quality and the feature size were studied successfully. Theoretically, it was seen that for our pulse width (laser pulse duration), repetition rate and pulse 
energy, the dots arrays spacing increased with the life time of the molten state $\tau_{\mathrm{L}}$ which equal to laser pulse duration, and laser fluence $\emptyset$, while other parameters did not play as decisive a role. It is also the first time that a low repetition rate nanosecond laser has been demonstrated for real world industrial application for laser fabrication in nano scales. Low pulse energy of $10 \mathrm{~K} \mathrm{Hertz}$ achieved acceptable quality of dots shape, and pattern quality is demonstrated during the experiments can be considerable when applying for industrial usage. Chapter 7 displays study for the influence of nanosecond laser parameter at second harmonic generation (SHG) wavelength of $532 \mathrm{~nm}$ for direct grating writing in common optical path setup. Also in Chapter 7 , the advantage of using common optical path configuration for laser interference and employed this technique for grating fabrication using femtosecond laser system have been demonstrated. Finally, Chapter 8 summarizes the results obtained from this research work. Also, the achievements and applications of the proposed patterning technique using the low repetition ratelow power nanosecond laser system are discussed. This Chapter also includes suggestions for further research that can be carried out. 


\section{CHAPTER 2 \\ LASER ABLATION MECHANISM}

\subsection{INTRODUCTION}

The production efficiency, continuous requirement for miniaturisation, and low cost MEMS, optical cables and other electronics parts, incessantly requires the development of industrially viable, efficient, and precise laser fabrication techniques with high resolution. Initially removal of material from the surface of metals and semiconductors following irradiation with nano, or femtosecond laser pulses are commonly called laser sputtering or laser ablation.

Nanosecond fabrication technology that used Diode Pumped Solid State laser uses a direct write method, which provides faster setup. The Nd: YAG and Nd: YLF lasers are the most common solid state lasers in industry. Different types of Nd lasers are currently employed. Nd laser can be frequency doubled, tripled or quadrupled through harmonic generation. Comparison of ablated structures fabricated by laser pulses of different pulse widths proposes that a higher structuring accuracy can be acquired with the use of short laser pulses to reduce the effect of (HAZ) [6]. The ablation mechanism is dependent on the properties of the sample material [7].

This Chapter focuses on the fundamental physical mechanisms and characteristics of laser-matter-interaction. It's valuable to refer that numbers of investigation has shown that the mechanism of laser ablation is determined by pulsewidth. Depending on the pulsewidth regimes, the mechanism can be thermal or non thermal in nature. However, the physics of laser matter interaction that leads to material breakdown and ablation differ with short and long pulses [7-12]. The next section will enrich best understanding for the benefits of laser ablation, laser matter interaction mechanisms both for nanosecond and femtosecond pulsewidth and it significance. 


\subsection{NANOSECOND LASER ABLATION}

Ablation using nanosecond laser pulses is difficult because it complicated mechanisms. This advantage is exploited by the time have been widely used, in particular, for material removal and micro fabrication [13-16]. In principle, any type of nanosecond laser with intensities above the ablation threshold could potentially be utilized as the light source for silicon machining. Figure 2-1, shows long pulse, laser-matter interaction which displays whole view of ablation spot and it subsequent resultants. There are many nanosecond lasers available, with repetition rates varying from a few 10 's of $\mathrm{KHz}$ to a few $\mathrm{Hz}$, having a maximum average per pulse intensity from a few $\mathrm{GW} / \mathrm{cm}^{2}$ to up to a thousand $\mathrm{GW} / \mathrm{cm}^{2}$ and pulse lengths from a few nanoseconds to a few tens of nanoseconds. The efficiency of laser pulse utilization in terms of removing material is an important aspect that needs to be known before choosing laser sources. The physical process of laser-silicon interaction in the nanosecond time regime is a complicated process, involving many aspects of laser-matter interaction. Relative to [17] states, the most fundamental feature of the interaction in the nanosecond regime are heat diffusion into the surrounding material. In other words, the process is thermal in nature involving direct vaporization [18]. The heat diffusion affects a large area. The heating and subsequent cooling waves in this region cause mechanical stress and microcracks, or sometimes macrocracks, which may propagate deeper into the material and cause premature device failure. Finally, the heat diffusion is associated with creation of shockwaves which can damage nearby device structures or split into multilayer materials. The more energy deposited into the micromachining process, the stronger the associated shockwaves. With long pulse lasers, the shot to shot reproducibility is poor. 


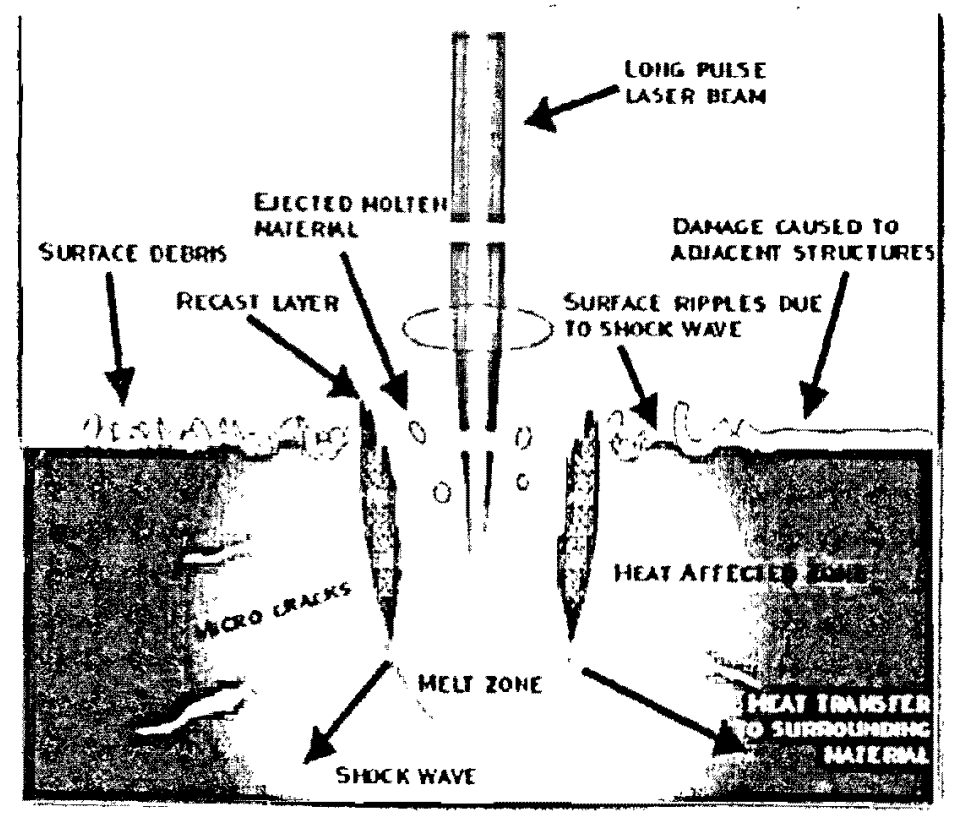

Figure 2-1: Long pulse, laser-matter interaction, displays whole view of ablation spot and it subsequent resultants [17]

By way of [18], in nanosecond lasers, the mechanism involves direct vaporization. The laser beam deposits energy in a shallow layer close to the surface. The surface reaches thermal equilibrium and some of the heat conducts to the material surrounding the laser spot. A molten layer forms and continuous application of the laser vaporizes the substrate. Material is removed by melt expulsion aided by a locally high vapor pressure. The material spatters around the irradiated area and solidifies as residue in the surrounding area. The thermal effects create a thick (HAZ). Shockwaves generated by the explosive change of phase can cause mechanical stress in the material. The formation of liquid and vapor phases around the spot interfere with the precision of the laser machining by deflecting the beam. Laser machining with nanosecond laser pulses lead to a large heat affected area and a large splatter zone for expulsed material. Heating 


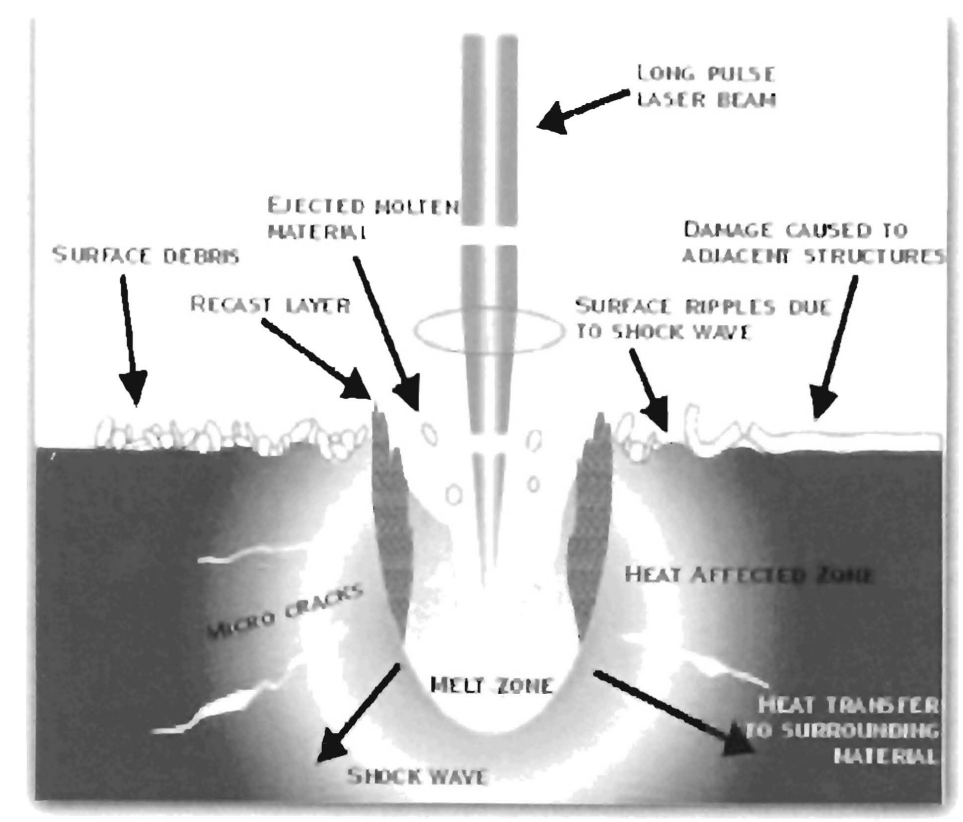

Figure 2-1: Long pulse, laser-matter interaction, displays whole view of ablation spot and it subsequent iesultants $[17]$

By way of [181. in nanosecond lasers, the mechanism inrolves direct vaporization. The laser beam deposits encrgy iis a shallow layer close to the surface. The surface reaches thermal squilibrium and some of the huit conducts to the material surrounding the laser spot. A molten layer forms and continuous application of the laser vaporizes the substrate. Material is removed by melt expulsion aided by a locally high vapor pressure. The material spatters around the irradiated area and solidifies as residue in the surounding area. The thermal effects create a ihick (HAZ). Shockwaves generated by the explosic change of phase can cause mechanical stress in the material. The formation of iiquid and vapor phases around the spot interfere with the precision of the laser machining by deflecting the beam. I aser machiring with nanosecond laser pulses lead to a large heat affected area and a a: ge splatter /une for expulsed material. Heating 
of metal targets by long laser pulses has been a subject of wide range of both experimental and theoretical studies $[7,8,37]$.

Herein this research work that executed by nanosecond laser, the ablation mechanism carried as a thermal effect. Furthermore the ablation is performed somewhat below the single shot ablation threshold, still some (HAZ) and surface morphology can be seen, which can be ascribe to a thermal process because the melt expulsion occurrence in the nanosecond regime. This would indicate that electronic melting is occurring and that ablation happens by homogeneous nucleation of gas bubbles in the liquid phase $[18,36]$.

\subsection{FEMTOSECOND LASER ABLATION}

Femtosecond laser pulses provide an attractive choice as it provides a way to obtain very clean removal of materials. The laser pulses illuminate the material for only a short period of time which in turn does not allow the heat deposited into the silicon to dissipate away from the work spot because the laser puise duration is shorter than the heat diffusion time. The peak power delivered by femtosecond lasers is extremely high and easily reaches hundreds of TW/ $\mathrm{cm}^{2}$ range at the work spot. The high power density allows for machining of very hard materials such as Molybdenum and Rhenium. In addition, as [17] mentions, the amplitude of the electromagnetic field corresponds to the peak power of the laser. Ultrafast lasers generate tremendous peak power. As such, the laser pulse can knock free the "bound" electrons in the sample. Per [18] depicts, the ablation is a direct solid-vapor transition. The timescale is to the first order, sufficiently short to ignore all thermal effects and hydrodynamic motion. The minimal (HAZ) is proof of this phenomenon. This leads to a clean cut with no visible collateral damage as shown in Figure 2-2. 


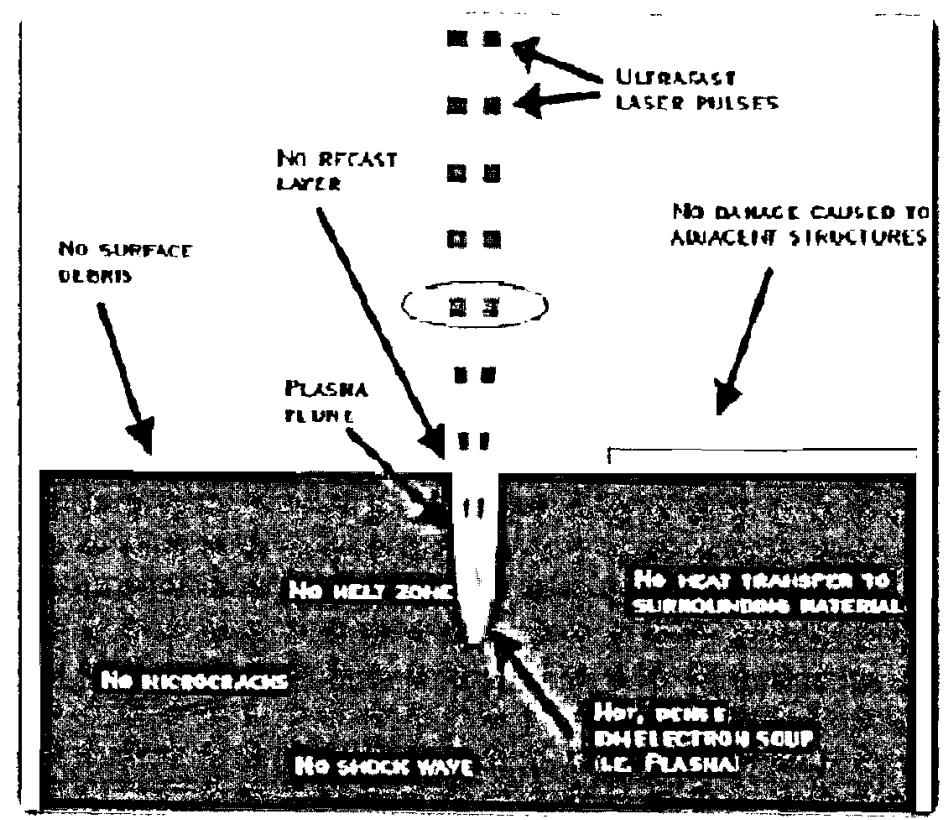

Figure 2-2: Short Pulse Laser-Matter Interaction [17]

In respect of the Ultra short lasers, the pulsewidth is too short for the heat to be conducted away from the surface during illumination. In this regime, the laser beam deposits its energy into the surface of the material, excites electrons to the conduction band and generates an excess of electrons. The laser pulsewidth is shorter than the electron relaxation time, which is on the order of picoseconds. By the time the laser beam is turned off, the system is out of equilibrium as the electrons are at a much higher temperature than the ions $[19,20]$. The hot electron relaxation dynamics in metals after the excitation by a femtosecond laser pulse has been intensively studied during the last decade $[38,39,40]$. The depth of the optical absorption determines the thickness of the heated layer and is independent of the pulsewidth [21]. One indication of this phenomenon is the absence of an observable heat affected zone. Author, Harzic et al, did not observe a (HAZ) in femtosecond ablation of Aluminum, but concluded that the (HAZ) was smaller than their observational limit of $2 \mu \mathrm{m}$ [22]. Another theory is that heating can occur electronically via fast 
Auger recombination [18]. The presence of amorphous silicon in certain experiments indicates rapid thermal effects because amorphous silicon forms from rapid cooling of liquid silicon [23, 24]. In the nanosecond timescale, where thermal melting occurs, melt expulsion by vapor pressure "pushes" molten material above the surface, leaving behind an amorphous ring [25]. Ablation would occur by a homogenous nucleation of gas bubbles at the surface causing an explosion and expelling of material [26]. Third, the mechanism may be delayed Auger recombination $[18,27]$. Here, heating of the substrate is a two step process, the first involving direct heating of the substrate by the laser pulse and the second by indirect heating from recombination effects [28]. Since the electron relaxation timescale is in the order of $1-10 \mathrm{ps}$. [29], the femtosecond laser beam is off by the time most of the heating occurs in the substrate. A fourth model is of plasma annealing $[18,30,31]$ which explains a change in structure via a non thermal annealing process. Once thermalization is complete, the material is molten and is then carried away by the hydrodynamic forces [32]. Some research indicates a combination of thermal and non thermal effects [18]. At higher fluences, melting occurs through ultrafast, non thermal effect. Above the ablation threshold, material is removed $[18,33]$. In metals, it has been shown that the energy not used in expelling material will induce a liquid phase leading to an amorphous or polycrystalline phase during solidification $[18,29,34]$. There has also been report on the oxidation of the ablated hole in addition to amorphization $[18,35]$.

\subsection{MECHANISMS IN LASER ABLATION PROCESS}

The principle of laser matter interaction is actually very hard to understand and includes full variety of different phenomenon, underlying the different aspects of laser matter interaction. In metals, the absorbed energy is transferred into the material by electrons or either by phonons 
in non metals. The energy relaxation time in metals is very fast $\sim 10^{-14} \mathrm{~s}$, although, in non metals, it is much slower of $\geq 10^{-12} \mathrm{~s}$, [41]. Laser matter interaction mechanisms, basically can explain by the ablation stages that would be, absorption and ionization, energy relaxation, heat transport, the Laser generated plasma, the melt flows in laser ablation, and shock wave phase. In non-metallic materials, the highest energy level occupied by an electron "in the valence band" is separated by an energy gap "the band-gap" from the lowest energy level that is unoccupied "in the conduction band". In femtosecond duration laser pulses, photo ionization during the leading edge of the laser pulse provides seed electrons for avalanche ionization [42]. Initially the electron density grows through avalanche ionization until the plasma frequency of the electrons in the conduction band approaches the frequency of the incident laser radiation (the "critical" plasma density). At the end of the laser pulse shot, basically there are hot electrons and a cold lattice left. Second stage of laser matter interaction mechanism is energy relaxation phase; the laser radiation interacts primarily with the electronic states of the valence and the conduction bands. The time to deposit the energy within these states is determined by the laser pulsewidth. Thirdly, is heat transport stage, which in dielectrics and semiconductors is comparably low, since the electrons are not able to escape due to the charge separation force keeping the material neutral. However, even in the case of metals heat diffusion can be significantly reduced when ultrashort pulses are used, due to the strong non-equilibrium interactions between electrons and lattice. Fourth, is the laser generated plasma phase, which it characteristics depend on the material, the laser pulse length, wavelength and laser intensity as well as the ambient conditions. The region closest to the target is the central or core region where the plasma temperature is maximal and the plasma density are highest. Fifth phase is the melt flows in laser ablation; the laser ablating is classified as volumetric laser ablation and superficial laser ablation. Shorter pulse length will reduce the melt 
pushups and hence reduce the melt recast and therefore improve ablation quality. Finally, shock wave phase, a shock wave is produced by the expansion of the plasma plume, if the ambient environment for ablation is a gas, such as air. The thickness of the target and the shock changing velocity determine the travel time, and the travel time together with the initial velocity determine the shock attenuation and its pressure at the back surface.

Ablation mechanisms using nanosecond laser are becoming even more complicated, but this can be exploited to our advantage, in particular, for high-aspect-ratio, very clean pattern of silicon due to simple setup of common optical path configuration which demonstrated in Chapter 3 and 4, and fast fabrication of single step as well as single shot, which is experimentally demonstrated in Chapters 5,6 and 7.

\subsection{COMMON OPTICAL PATH CONFIGURATION}

Laser interference is a maskless patterning technology to produce periodic and quasiperiodic nanostructures. The optical interference pattern can be used to produce interferometer gratings. There are numerous interference path configuration approaches to generate fringes from one or more light beams. That task demands light source of high temporal and spatial coherence. The interference patterns can be arrays or matrices of laser beam lines or dots [95].

The expanded laser beam when split into two equal parts by wave front or beamsplitter, guide it by two or more mirrors to reflected. An interference pattern can form by using common optical path which direct and reflect two beams through single optical part to focus and superpose beams on the sample surface instead long separate paths. Hence common optical path configuration offers fringes pattern from two laser beams of same the energy distribution in interference pattern with true interference fringes and their contrast. Common optical path 
configuration can lower the vibration, when vibration is too large, the fringes may be disappear or arise with low contrast. Furthermore, employing common optical path configuration would be error free which may be caused by time delay between the two interfering waves through long optical path and each passing through different optical path.

This method is based on selective laser ablation of materials by two or more high power laser beams, which superimpose on the sample surface, and form an interference pattern. The energy distribution $I(x)$ of a two-beam interference pattern are given by Equation [90],

$$
I(X)=2 I_{o}\left[\cos \left(\frac{2 \pi x}{d}\right)+1\right]
$$

Where $I(X)$ is laser energy distribution for two interference beams, $I_{0}$ is the intensity of laser beam, and $d$ is the period (fringes distance). The grating period is changed by varying $\theta$ or the incident wavelength.

\subsubsection{BEAMS INTERFERENCE FORMATION}

Laser radiation is supply coherent light with a suitable wavelength and coherence length. It can be performed by a single laser or a laser with amplifiers. Solid state lasers system is common for laser ablation and surface structuring; it can be a good selection for direct writing patterning. It can choose lasers according to their requirements especially in laser interference. Interference phenomena have been the means of establishing the wave nature of light and have found considerable practical applications in interferometer, metrology and spectroscopy. Later on the interference phenomena have been utilized for the surface fabrication of materials in a spacious range of applications such as micromachining and biomedical applications. Laser interference processing is a comparatively new technique finding raised utilization in the field of 
materials surface processing. Laser interference technique includes splitting of a laser beam by a beam splitter followed by superposition of the beams by single prism or set of mirrors to generate interference patterns. Therefore the interference pattern that produced shows matchless intensity variation which can be employing for periodic material surface patterning. The typical interference pattern produced by the superposition of two laser beams is shown in Figure 2-3. A small angle between the beams will produce a widely spaced fringe pattern "large d", whereas a larger angle will produce a fine fringe pattern [92].

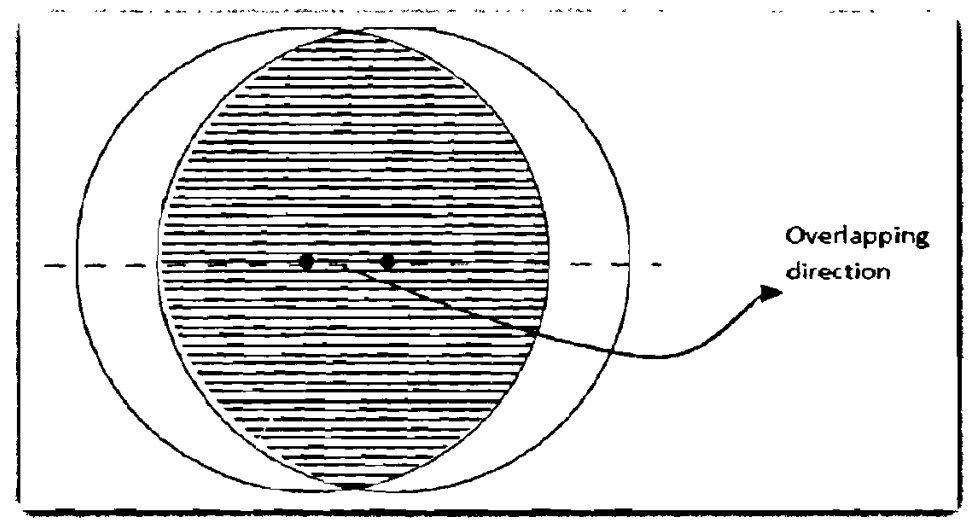

Figure 2-3: Two laser beams interference pattern

\subsubsection{FRINGES GENERATION}

If two pulses meet on sample surface, they interfere and form fringes. Common optical path configuration interference techniques employ the modulation of laser intensity that occurs when a coherent beam is split into two beams and subsequently interfere with each other at the substrate surface. Only highly periodic structures, such as gratings and dots arrays, can be fabricated by interference method. Interferometric grating patterning is based on the formation of stable and uniform interference fringes. That task demands light source of high temporal and 
spatial coherence. Typically the interference induces by intense ultraviolet laser radiation of $\mathrm{Nd}$ : YAG laser and femtosecond laser. The interference of the two beams produces a sinusoidal intensity variation at the surface. The spatial frequency of this laser pattern is determined by the angle between the beams. The intensity distribution of the interference patterns exposes materials with a pitch of sub-wavelength of the interfering light. When using such radiation to interact with materials, feature sizes down to a fraction of the laser wavelength can be created. This technology provides a way for submicron and nano patterning periodic and quasi-periodic patterns that are spatially coherent over large areas.

\subsubsection{OPTICAL (HOLOGRAPHIC) INTERFERENCE}

High efficient optical gratings which is called holographic grating can be obtained by applying the interference pattern of two coherent laser beams in common optical path configuration. The grating spacing is governed by the angle between the two incident beams. As shown in Figure $2-4$, the holographic grating fabrication by interfere two laser beams, the grooves are created at simultaneous time.

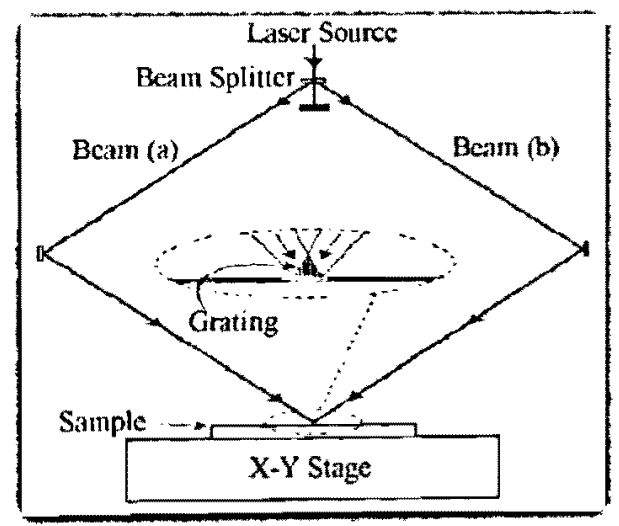

Figure 2-4: Grating fabrication by optical interference of two laser beams in common optical path configuration 
Interference holography is a common tool for making diffraction gratings and the principle of interference holography is simple. Two beams of laser interfere and produce interference fringes parallel planes of high and low laser intensity. A useful analogy is to visualize two expanding circular water waves excited possibly by the dropping of two stones into a quiet pool. Where the two waves intersect a stable interference pattern called a standing wave will appear. Furthermore Interference techniques employ the modulation of laser intensity that occurs when a coherent beam is split into two beams that subsequently interfere with each other at the substrate surface. Only highly periodic structures, such as gratings, can be fabricated with this technique. The relief gratings produced by these interference techniques are classed as thin film holograms, so the technique is also called holographic fabrication. The interference of the two beams produces a sinusoidal intensity variation at the surface. The spatial frequency of this laser pattern is determined by the angle between the beams. The spacing between intensity maxima is given by,

$$
d=\frac{\lambda}{2 n \sin \theta}
$$

Where, $n$, is the index of refraction of the machining surroundings. The grating period (d) is changed by varying $\theta$. There are two important components to the resolution of grating produced by this interference technique. The quality of a grating is generally better if it is machined at lower intensities; actually a long time machining at higher intensities can completely destroy a grating. The quality of a grating can be monitored during fabrication by regulate the laser beam intensity, which first increases with machined time. The maximum number of lines in a grating $(N)$ is determined either by the bandwidth or the source divergence which can represent by the Equations, 


$$
\mathrm{N}=\frac{2 \lambda}{\Delta \lambda}=\frac{2}{(\Delta \theta)^{2}}
$$

Where $\Delta \lambda$ is the bandwidth or wavelength spread and $\Delta \theta$ is the divergence angle. A special technique known as spatial frequency doubling is an alternative to regular interference techniques. Using $193 \mathrm{~nm}$ laser wavelength, $99.5 \mathrm{~nm}$ grating spacing has been achieved $[2,4,5]$.

\subsection{SUMMARY}

The complicated physical process of laser-matter interaction involves variety of different phenomena. The laser ablation mechanism which is the process of material removal upon the irradiation of the laser beam on the surface be reliant upon the properties of the sample material along with the laser pulsewidth. Aspects of laser-matter interaction are elaborated through the differences between short and long pulses. Long pulses such as nanosecond lasers, material removal process is through phase explosion where due to thermal accumulation over the surface, more (HAZ) and cracks occur. In ultrashort pulses such as femtosecond lasers, the ablation mechanism is mainly via solid to vaporization state of materials. In femtosecond laser matter interaction in terms of transferring laser energy into thermal energy of the target is that electrons and ions are not in equilibrium during the laser pulse. Result in strict machining of the sample without thermal damage and less cracks. Common optical path configuration is the most effective method of fringe generation by high intensity laser beams interference which imprint on substrate surface for grating and dot array fabrication. No errors can induced, no vibration because minimize the optics number that used. High efficient optical gratings which is called holographic (optical) grating can be obtained by employing the interference pattern of two coherent laser beams in common optical path configuration. 


\section{CHAPTER 3 \\ COMMON OPTICAL PATH CONFIGURATION FOR FRINGES GENERATION}

\subsection{INTRODUCTION}

Laser interference processing is a comparatively new technique finding increased utilization in the domains of extended-area surface processing of materials. In this Chapter, a brief study of the applications and the theory of laser interference phenomenon for materials surface patterning have been exhibited and discussed.

There are different focusing setups as well as there are long focusing paths which make the whole experiment setup not immunize for inherent vibrations. The concept of setup that used in this study is very short and common focusing path, its works on simplifies the optical setup. Subsequently after expanded the laser beam, it's reflected by a mirror of $100 \%$ efficiency and $45^{\circ}$ deflection angle to guide it through focusing scanning lens of $75 \mathrm{~mm}$ positive focal length. The collimated beam was split into two orthogonal beams of equal intensity by a non polarizing beamsplitter NPBS, as shown in Figure 3-1. The intensity of two laser beams can be control through tuning and adjusting the $\lambda / 2$ waveplate that displayed in Figure (4-1). A custom-made crystal prism (CMP) $(2.5$ in $\times 1$ in $\times 1$ in) was used to reflect and redirect two laser beams. Subsequently the two beams have interfered on the sample surface and the interference fringes have been generated. 
Both side surfaces of custom-made prism is coated with $45^{\circ}$ mirror coating to restrain the intersection angle $\theta$ and fixed to certain direction at $90^{\circ}$. The two beams will interfere at focal point which located at the sample's surface and get the interference fringes imprinted and generate the grating, as reported by [57]. In this case the laser must have a few conditions in order to achieve a correct and spatial interference fringes, such spatial coherence and sufficient temporal coherence for path length mismatch.

\subsection{COMMON OPTICAL PATH CONFIGURATION \\ 3.2.1 TECHNIQUES METHODOLOGY}

The conventional gratings techniques involve complex setup which includes path length more complex. It involves more optical components for optical delay that prevent the difference between two optical paths, as well as long pathway that requires more energy and additionally energy loss through whole optical components. The optical path difference between the interfering beams is determined by the difference in the length of the interferometer paths. The optical path difference must be less than the coherence length to maintain the temporal coherence. The optical path difference also determines how well defined the pattern is. If one path of the interferometer is shorter than the other, the beam from the shorter path will arrive at the sample surface first thus initiating the surface modifications at the sample surface. For such a case, the interaction time between the beams will decrease.

Most of the laser interference setup procedures, optical apparatus such as beamsplitter are used which divide an incident laser beam into two by partly transmitting and reflecting a laser beam. In a simplified arrangement, a beamsplitter comprised two triangular glass prisms which 
are joined together at the base using appropriate resin. In other arrangements, thin films deposited on glass surfaces which improve the reflectivity can be used as beamsplitter [90].

\subsubsection{CUSTOM MADE PRISM (CMP)}

In this study an original setup for surface patterning by laser interference have been designed from commercially available optical elements. The setup is comprised of $\mathrm{Nd}$ : YAG laser, non polarization beamsplitter and custom-made special prism. The intensity of the two beams can be controlled by rotating the $\lambda / 2$ waveplate. The prism is an optical slab, the two side surfaces were polished then coated with $45^{\circ}$ coating twice to ensure precise reflecting tol 064 and $532 \mathrm{~nm}$ wavelengths. The two orthogonal beams (beam "a" and beam " $b$ ") reflect and automatically interfere at the focal point at sample surface. The interference fringes with high intensity imprinting and generate surface patterning on the sample. This prism insures that the two incident beams travels the same lengths in common optical path. The geometry of the interference patterns formed by the superposition of two coherent and linearly polarized beams depends on the wavelength and the angle between the beams. The intensity distribution $\mathrm{I}(\mathrm{X})$ resulting from the superposition of two linearly polarized beams with their $\mathrm{E}$ vectors in the $\mathrm{x}$ direction as expressed by Equation 2-1 [90],

The interference of the two beams generates only one dimensional periodic pattern. While two and three dimensional periodic patterns such dots array pattern can be obtained by increasing the number of beams. In that case, the intensity distribution resulting from the superposition of four beams is given by,

$$
I(X, Y)=4 I_{0}\left[\cos \left(\frac{2 \pi x}{d}\right)+\cos \left(\frac{2 \pi y}{d}\right)\right] \wedge 2
$$


Where $I(X, Y)$ is laser energy distribution for four interference beams, $I_{o}$ is the intensity of laser beam, $d$ is the fringes period. Typical arrangement for materials processing using two laser beams interference employing custom made prism (CMP) is shown in Figure 3-1. The various elements of this arrangement are the laser source, mirrors beamsplitter and custom made prism.

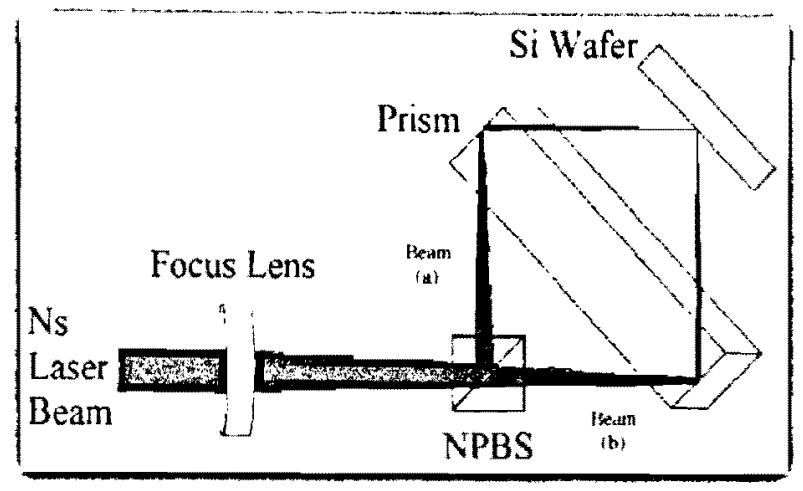

Figure 3-1: The schematic diagram of common optical path configuration setup using custom-designed prism

The processing advantage of using this method is common optical path configuration with automatic alignment for direct fabrication. The setup involves few optics and no adjusting components. The simple configuration means no special skills are required to operate and maintain the system. The only error in the common optical path length comes from the parallelism of the two mirror surfaces, which can be controlled by tightening the tolerance. Moreover the special custom-made prism is creative method used for beams interference. The special manufactured prism has two side surfaces which flatted in an extreme accuracy to certain meticulously angle of $45^{\circ}$, it's coated for perfect light reflecting with a least energy loss. The special-made prism's surfaces were made within double coating for both 1064 and $530 \mathrm{~nm}$ wavelengths on the same coating mirror. The coating processing for this kind of prism mirrors was obtained by two steps, at the time many of cheap optical parts coating in single shot. The 
two side surfaces of the (CMP) coating was performed by two steps; polishing and coating twice with time roughness flatness wave plate $\lambda 25$ and then measured by optical microscopy to ensure the flatness accuracy.

\subsubsection{BEAM SPLITTING}

Beam splitting is required to acquire separate coherent beams for laser interference technique. Two beams interference gives linear fringes which are attractive for a number of applications such gratings fabrication, while multi beam interference is needed for fabricate two or three dimensional structures such dots arrays pattern. It is worthwhile to be able to select the number of output beams to generate different patterns in selective materials. For a multi beam interference system, the select of beam splitting methods varies with the number of beams, flexibility, pattem size, laser wavelength and power. In this study a non polarizing beamsplitter NPBS which diverge an incident laser beam into two equal intensity beams by partly (50\%) transmitting and reflecting a laser beam. In a simplified arrangement, a beamsplitter comprised two triangular glass prisms which are joined together at the base using appropriate resin.

\subsubsection{LASER IRRADIATION}

The role of laser irradiation is to supply coherent light with a suitable wavelength, power and coherence length. It can be performed by a single laser or a laser with amplifiers. Solid state lasers of Nd: YAG system is common for laser ablation and surface structuring; it can be a good selection for direct writing patterning. Excimer lasers are also appropriate for high instant power applications, and perfect for direct writing. It can choose lasers according to their requirements. 


\subsubsection{THEORY OF INTERFERENCE}

As to a beam of laser, if divide by an appropriate device such beamsplitter or by the division of wave front into two or more beams which are afterward superposed, the energy intensity in the area of super position exhibits the matchless variation. It was found that energy intensity in the area of beams superposition alters from point to point between the maximum, "passing the sum of intensities in the beams" and the minimum "possibly zero". This superposition of two or more beams is referred as interference. When two coherent beams of equal intensity and equally polarized intersect each other. Interference patterns are generally formed in the region of intersection if both sets of light are of the same wavelength. The beams coming from different sources are reciprocally incoherent and no interference is usually observed under common experimental condition. Anyway, if the two beams originate from the same source, the fluctuations in the beam are generally correlated and the beams will be completely or partially coherent. The superposition of these coherent beams that originating from the same source leads to interference patterns. There are two methods for acquiring coherent beams from a single laser source; division of amplitude and division of wave front. Initially, a beam is divided by partly reflecting surfaces where a part of laser is reflected and the other part is transmitted such beamsplitter. In the other method, a beam is divided by passage through adjacent apertures [91]. The interference patterns, generated from two coherent beams of laser radiation, give arrays of laser beam lines. Figure 3-2 shows section X-X of fringes for two laser beams interference and fringes are formed. 


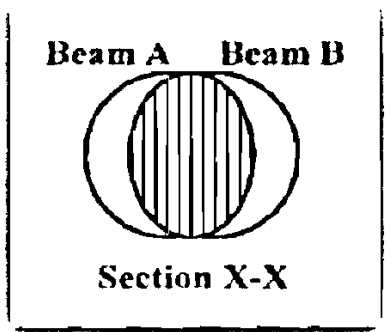

\section{Figure 3-2: section $x-x$ of two laser beams interference that shows fringes form}

\subsubsection{FORMATION OF LASER INTERFERENCE PATTERNS}

Interference phenomena have been the means of establishing the wave nature of light and have found considerable practical applications in interferometer, metrology and spectroscopy. Later on the interference phenomena have been utilized for the surface fabrication of materials in a spacious range of applications such as micromachining and biomedical applications. Laser interference processing is a comparatively new technique finding raised utilization in the field of materials surface processing. Laser interference technique includes splitting of a laser beam by a beam splitter followed by superposition of the beams by single prism or set of mirrors to generate interference patterns. Therefore the interference pattern that produced shows matchless intensity variation which can be employing for periodic material surface patterning. The typical interference pattern produced by the superposition of two laser beams is shown in Figure 3-3. A small angle between the beams will produce a widely spaced fringe pattern, whereas a larger angle will produce a fine fringe pattern [92]. 


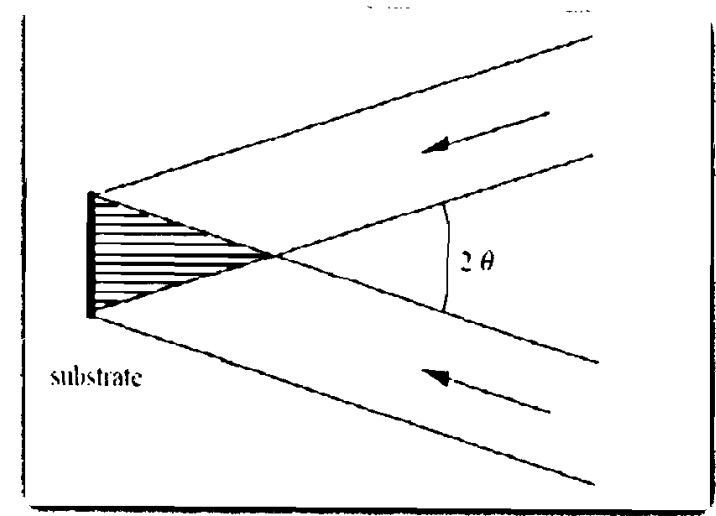

Figure 3-3: displays formation of interference fringes by two laser beams

\subsubsection{CREATION OF GRATINGS PATTERN}

There is a wide variety of optical systems that may be used to bring together two beams of light in such a way that will generate interference fringes. In all cases the two interfering beams are generated from the same light source in order to ensure coherence [93]. The two beams may be acquired either by the division of amplitude such beamsplitter which reflects a fraction of the laser intensity of the whole beam and transmits what is left after absorption. The other method may be obtained by the division of wave front, in which case one half of the wave front pursues a different optical path to the other half. Both beams will intersect at the focal point at sample surface and give fringes pattern of high contrast [94]. The high fringes intensity will translate, inscribe on the surface and generate gratings like comb patterns. Figure 3-4 shows grating pattern which arise from fringes that induced by interfering two coherent high intensity laser beams. The smaller the angle between the interfering beams, the larger is the spacing in the pattern. 


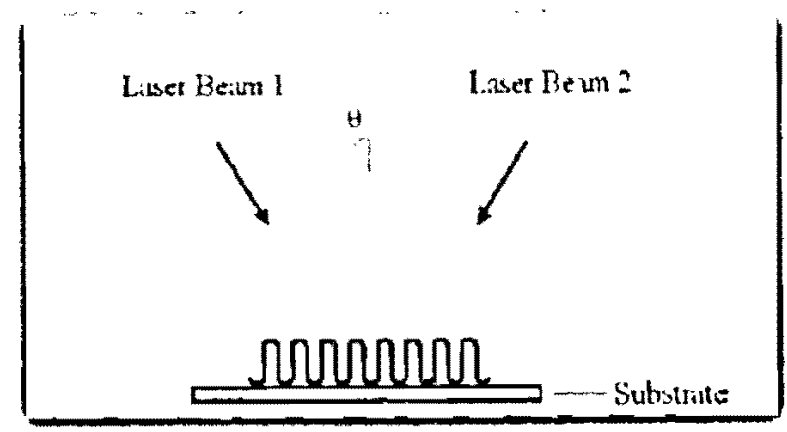

Figure 3-4: shows surface patterning of grating which arise by the interaction between high intensity fringes with the sample

\subsection{SUMMARY}

In this Chapter the theory and application of fringes interference have been outlined. When two coherent liners polarized laser beam intersect through common optical path configuration, interference fringes pattern will arise that caused by two beams superposition. Common optical path configuration method offers beast result for high quality fringes as well as less energy loss. There are two methods to acquire two beams, either by the division of amplitude such beamsplitter which reflects a fraction of the laser intensity of the whole beam and transmits what is left after absorption. The other method may be obtained by the division of wave front, in which case one half of the wave front pursues a different optical path to the other half. Optical (CMP) was used in such common optical path configuration to guide and overlap the two laser beams. This prism insures that the two incident beams travels the same lengths in common optical path. The geometry of the interference patterns formed by the superposition of two coherent and linearly polarized beams depends on the wavelength and the angle between the beams. Both beams will intersect at the focal point at sample surface and give fringes pattern of high contrast. The advantages quality is very uniform, simplifies the setup, self alignment, equal beam intensity, and immunizes the system to extraneous and inherent vibrations. 


\section{CHAPTER 4 \\ EXPERIMENTAL DETAILS}

\subsection{INTRODUCTION}

Commencement, the experimental setup that used for this study has been made to ensure getting large machining area for stable fringes and low errors. The setup was represented by resplendent innovation by common optical path configuration which uses CMP. All experiments were carried out in ambient atmosphere. The experimental setup and related parameters, like interfering beams arrangement, laser beam spot size and the laser beam polarization are discussed. Also, all experiments were conducted using a low power, low repetition rate of $\mathrm{Nd}$ : YAG nanosecond laser system capable of delivering a maximum output power of $10 \mathrm{~W}$. The fundamental laser wavelength is $1064 \mathrm{~nm}$ with liner polarization and pulse duration of $20 \mathrm{~ns}$ was adopted in forming surface submicron grating and nano dot arrays. The experimental results were carried in the Micro and Nano Fabrication Research Lab, Mechanical engineering department, at Ryerson University, Toronto, Canada.

\subsection{EXPERIMENTAL SETUP DETAILS}

Figure 4-1 shows a schematic drawing of optical setup configuration for the surface patterning. The nanosecond laser system used in the experiment is a diode pumped, Nd: YAG system capable of producing average output power of $10 \mathrm{~W}$ with a fundamental central wavelength of $1064 \mathrm{~nm}$ and repetition rates ranging between $10 \mathrm{KHz}$ and $100 \mathrm{KHz}$. The laser beam is polarized to either $\mathrm{P}$ or $\mathrm{S}$ polarization. The optical $(1 / 2 \lambda)$ waveplate is used for modifying 
the polarization of light. The laser beam was collimated and expanded by two (UV) fused silica (Plano concave and convex) lenses of $-50 \mathrm{~mm}$ and $+400 \mathrm{~mm}$ focal length.

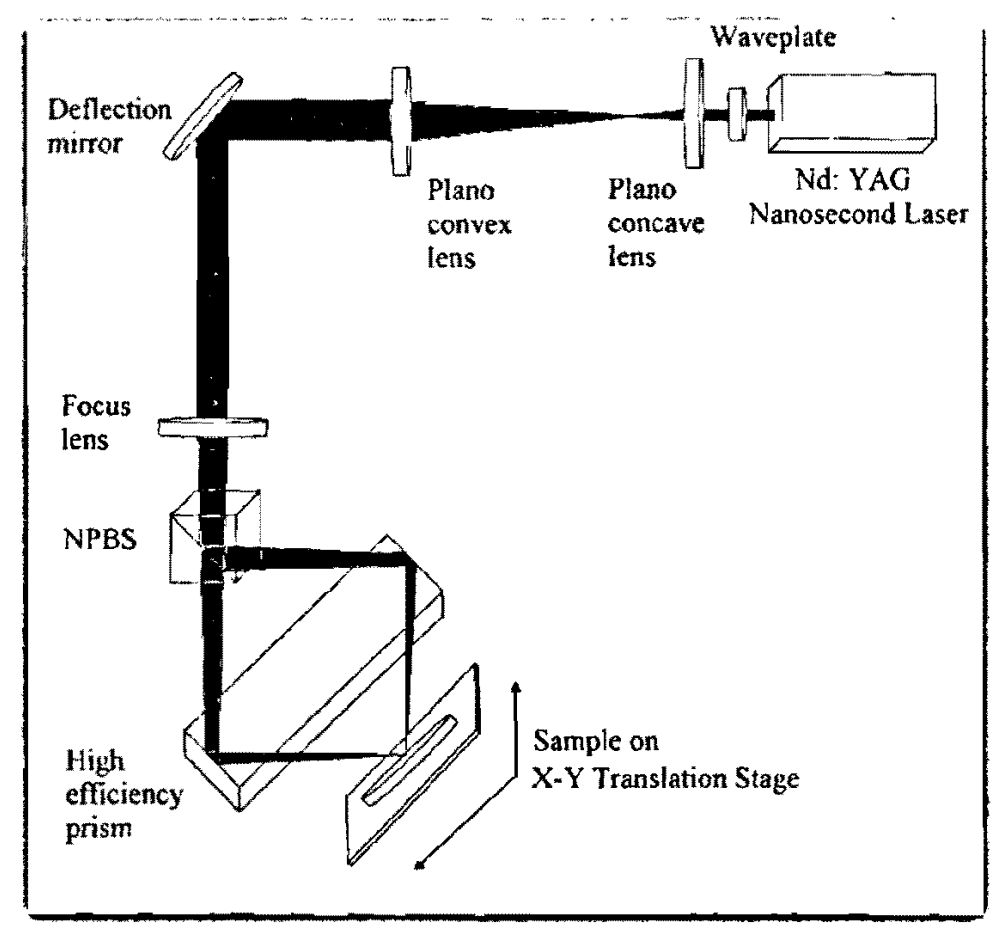

Figure 4-1: The schematic diagram of the experimental setup for surface patterning using common optical path configuration.

As well as Figure 4-2 express real picture of optics configuration sit on the smart table inside the laser fabrication lab, Ryerson University. 


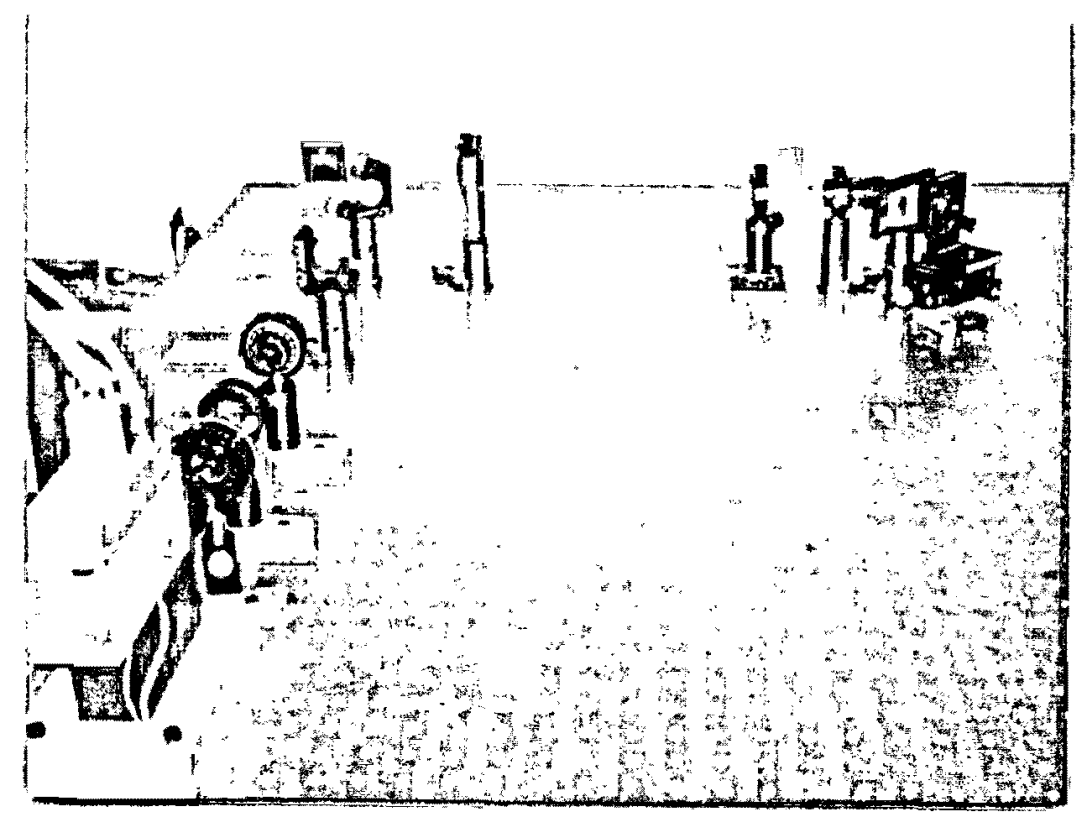

Figure 4-2: picture of optics configuration inside the laser fabrication lab, Mechanical Engineering, Ryerson University-2009 Toronto

The laser beam reflected by the mirror and passes down then split into two orthogonal beams of equal intensity. The diverging laser beam was then made to pass through the common path configuration described in the below section. The lens that was used to focus the laser beam must have focal length long enough to provide sufficient work space to accommodate the optical setup for the common path configuration. The mechanical stage was used to transport the sample in the direction parallel to the fringes.

In this configuration, the incident angle $\theta$ is $45^{\circ}$. 


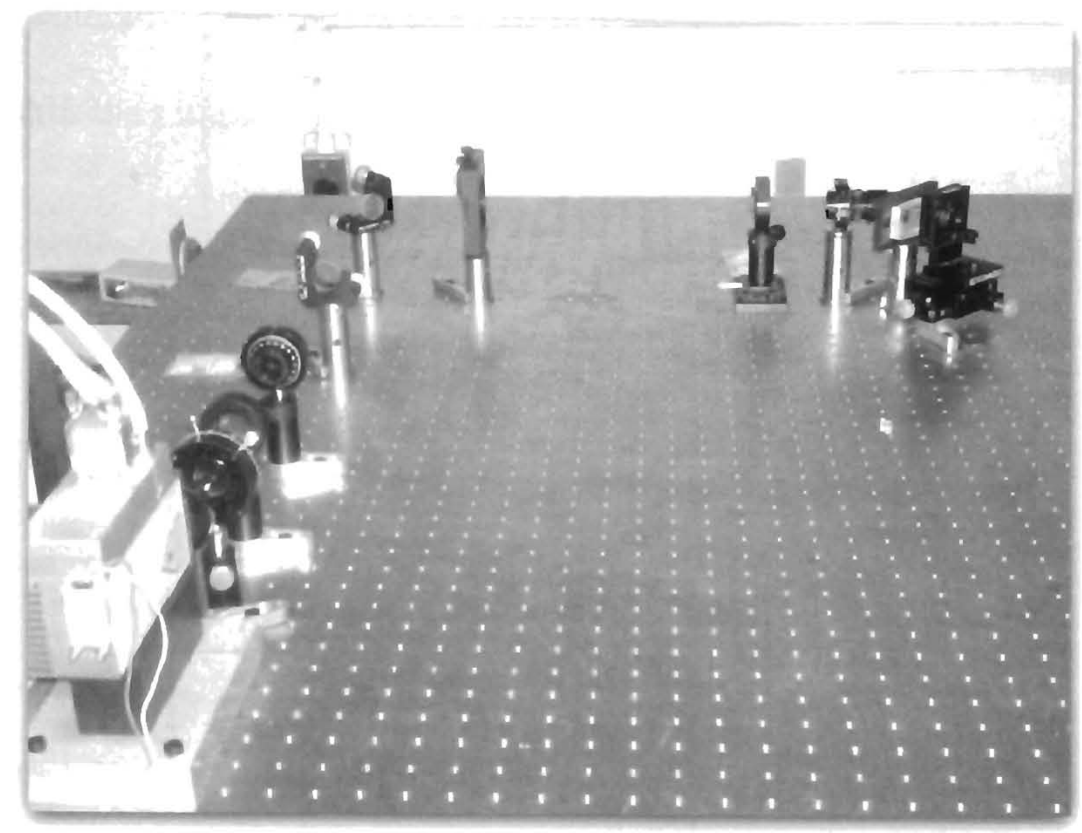

Figure 4-2: picture of optics configuration inside the laser fabrication lab. Mechanical Engineering,

\section{Ryerson University-2009 Toronto}

The laser beam reflected by the mirror and passes down then split into two orthogonal beams of equal intensity. The diverging laser beam was then made to pass through the common path configuration described in the below section. The lens that was used to focus the laser beam must have focal length long enough to provide sufficient work space to accommodate the optical setup for the common path configuration. The mechanical stage was used to transport the sample in the direction parallel to ihe fringes.

In this configuration the incident angle $\theta$ is $45^{\circ}$. 


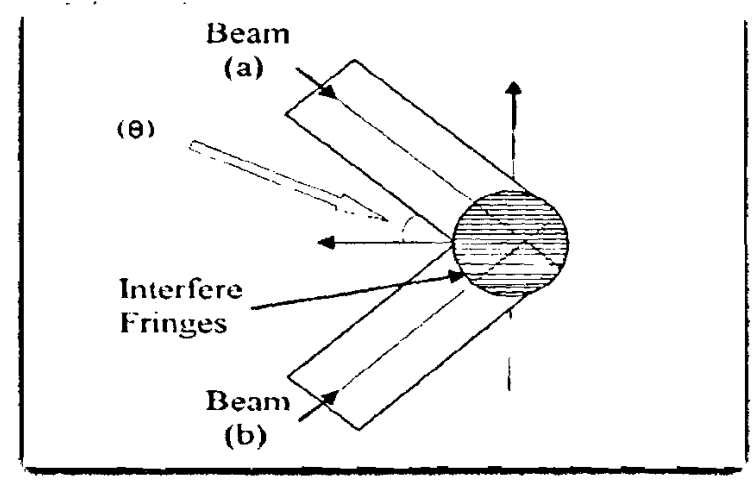

Figure 4-3: The schematic diagram of the interfering spot area (A) shows the bonded angle between $x$ axis and single beam

\subsection{THE PROCESSES PARAMETERS}

\subsubsection{SPOT SIZE}

Laser beam quality is quantified by the beam parameters, which one of them is the beam width or (spot size). After laser beam expansion using multi optics in the optical configuration setup, the laser beam should go to the waist size (spot size) before hit the machining sample in order to increase the laser intensity and efficiency. That can obtain by using focus lens with suitable focal length to give enough distance between it and the sample. Practically, the way to know the size of the focused beam spot, a single spot can be machined on a thin metal film, with pulse energy well above the material threshold. The size of the machined spot, measured using a Scanning Electron Microscope, bestows the focused beam size. Whereas theoretically, the spot size $\left(d_{0}\right)$ value of the firmly focused laser beam can be expressed as in Equation 4-1 [49],

$$
\mathrm{d}_{\mathrm{o}} \approx \frac{1.27 \lambda f}{\mathrm{D}}
$$

Therefore the spot size at wavelength of $1064 \mathrm{~nm}$ is calculated as $40 \mu \mathrm{m}$ and $20 \mu \mathrm{m}$ at $535 \mathrm{~nm}$. During the experiment the spot size may be more spacious caused by scatter and 
misalignment. Generally, smaller spot size results in higher laser fluency which need faster speed and control to shift the sample during the machining.

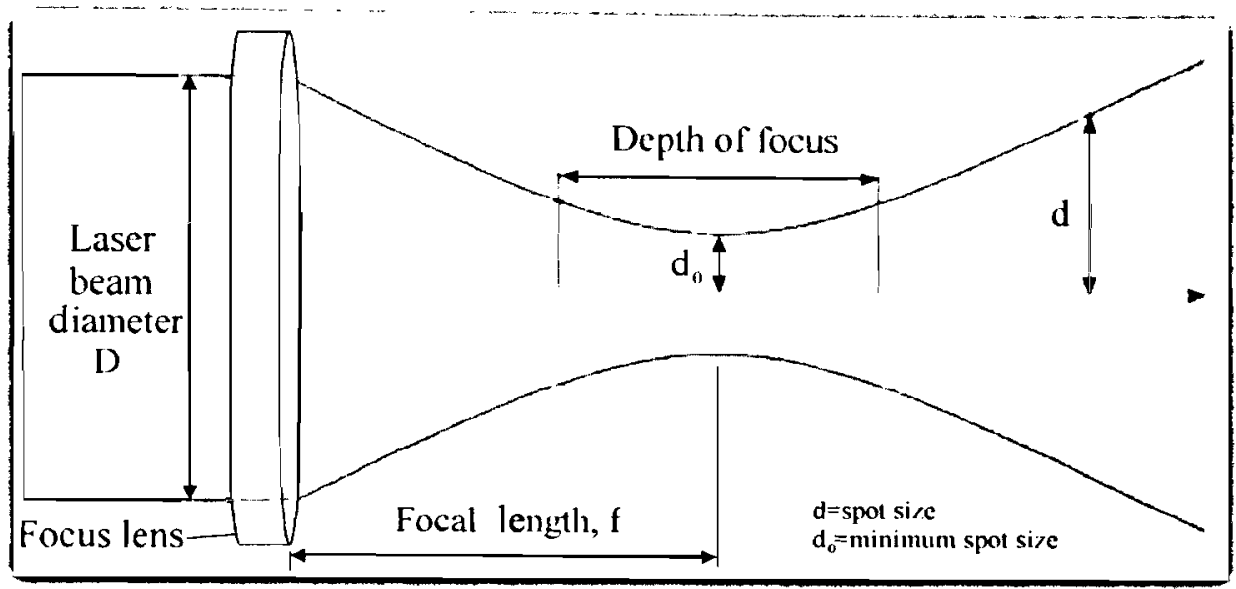

Figure 4-4: Laser spot size $\left(d_{0}\right)$ and depth of focus $\Delta f$, for laser beam using a convex focus lens

For a Gaussian beam propagating in free space, as shown in Figure 4-4, the spot size $d$ will be at a minimum value $d_{o}$ at one place along the beam axis, known as the beam waist. The distance between the plane of focus lens and beam waist known as focal length, $f$. The optimum machining requirement, it meet the condition of focus depth $\Delta \mathrm{f}$, which to reduced a focus spot size without sacrificing depth of focus. This is lead to preventing the loss of efficiency of laser beam without any losing of the beam intensity. It can be calculate the depth of focus (DOF) is given by Equation 4-2,

$$
\mathrm{DOF}=2.5 \lambda(f / D)^{2}
$$

Where, $\lambda$ symbolizes laser wavelength, $f$ symbolizes the effective focal length of used focused lens, and $D$ symbolizes the laser beam diameter at the output of the Plano-convex lens. In our setup and experiment, (DOF) was $1.66 \mathrm{~mm}$ which quite small that makes the interference 
procedure to be hard from first time and after a while gave us kind of challenging to possession such as these valuable results.

\subsubsection{POLARIZATION}

Micromachining laser fabrication of silicon efficiency is a beam polarization dependent process. There are two kinds of polarization, liner and circular polarization, which the liner in turn divides to $\mathrm{P}$ and $\mathrm{S}$ polarization. Using a half $(\lambda / 2)$ wave plate in front of the focusing lens, the polarization state of the beam can be converted to $P$ from $S$ and vice versa. In order to get a circular polarization the half $(\lambda / 2)$ wave plate can be replaced by quarter $(\lambda / 4)$ waver plate placed $45^{\circ}$ to the plane of incidence of the beam. The linear polarization states of the laser beam, that is to say $\mathrm{P}$ polarization, $\mathrm{S}$ polarization and circular polarization $\mathrm{C}$.

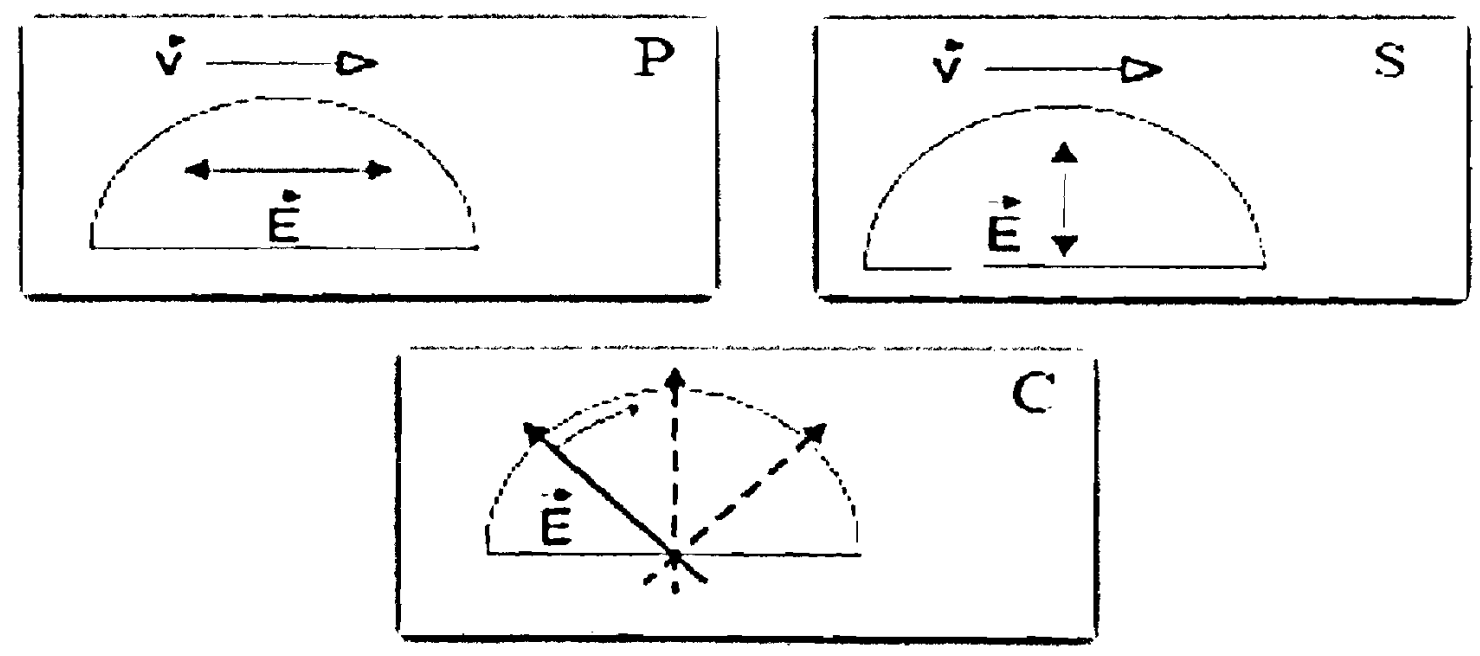

Figure 4-5: exhibits, basic definitions of $P, S$ and $C$ polarizations

Laser beam polarization refers to the electronic vector of the laser beam oscillates in a specific direction with reference to the plane of incidence of the beam. Furthermore in case of $P$ polarization, an electrical vector (E) is parallel to the beam velocity vector (V), and when 
electrical vector $(E)$ is perpendicular to the beam velocity vector $(V)$ will reference to $S$ polarization, as shown in Figure 4-5. The influence of the orientation of the polarization on machining efficiency and feature shape has been well studied for the laser micromachining applications $[50,51]$. Due to the different reflectivity at different incident angles, a linearly polarized laser spot has a higher intensity at the bottom of the cut in the direction perpendicular to the polarization.

\subsection{SILICON MATERIAL SPECIFICATIONS}

Silicon material predominates in micromachining area and therefore is selected as test samples for this study, also tested are gold thin films of thickness of $3000 \AA$ coated on silicon substrate and gold thin films of thickness of $1000 \AA$ coated on transparent substrates. Silicon is the most common semiconductor used in optoelectronic and microelectronics devices. It has band gap of $1.07 \mathrm{eV}$ which leads to efficient detection of visible light and conversion of sunlight into electricity. Silicon is produced in higher volume for lower cost than any other semiconductor and comes only second to oxygen in earthly abundance. Silicon is used in many forms (including crystalline, amorphous, and porous) in numerous optoelectronic and photonic devices. Silicon sample must adhere to strict mechanical, electrical and surface specifications. However, silicon has several defects, as silicon cannot be used to detect many important communications wavelengths; it is a poor light emitter and an indirect band gap material. A large research effort is directed at manipulating the properties of silicon to overcome these shortcomings. The low cost and easy integration with existing silicon fabrication techniques that drove researchers to find new forms and uses of silicon. Table 4-1 shows, some of single crystalline $\mathrm{Si}(\mathrm{C}-\mathrm{Si})$ parameters $[10,11,14,15,81]$. 


\begin{tabular}{|c|c|c|}
\hline Parameter & Value & $\underline{\text { Int }}$ \\
\hline The meltung pount $T_{m}$ & 1687 & $\mathrm{k}$ \\
\hline Specific heat $C_{F}$ & 0.7 & $\mathrm{Jg}^{-1} \mathrm{C}^{-1}$ \\
\hline Therwal conducturty $k_{\text {eq }}$ & 0.46 & $W \mathrm{~cm} \mathrm{~K}$ \\
\hline Reflectrity $R$ & 0.3 & - \\
\hline Band gap $e^{\prime}$ & 1.07 & $\mathrm{eV}$ \\
\hline Specific heat capacty $C$ & 2 & $\mathrm{Jcm}^{-5} \mathrm{~K}$ \\
\hline Specific heat of meltung $L_{m}$ & 4130 & $\mathrm{~J} \mathrm{~cm}^{-3}$ \\
\hline Surface tension coefficient $\sigma$ & 850 & $\mathrm{~m} \mathrm{Vm}^{-1}$ \\
\hline Liqud mass density $\rho$ & 2.52 & $\mathrm{~g} \mathrm{~cm}^{-3}$ \\
\hline Atomic weight $t_{r}$ & $28.086 \times 10^{-3}$ & kg mole \\
\hline
\end{tabular}

Table 4-1: C-Silicon specifications

\subsection{SUMMARY}

The experimental setup is designed with a minimum number of optical components; the technique can simplify the optical parts for a better fabrication process with fewer vibrations. The setup comprised common path optical configuration using (CMP) instead of using two reflecting mirrors to ensure easy beams interference for nanosecond laser. Laser parameters including wavelength, pulse duration, pulse energy intensity and circular beam shape with pure Gaussian pulse shape must be optimized to fulfill the requirements of precise tasks and to maximize the efficiency of laser power usage. No special skills are required to operate and maintain the system. The only error in the common path length comes from the parallelism of the two mirror surfaces, which can be controlled by tightening the tolerance. 


\title{
CHAPTER 5
}

\section{SURFACE PATTERNING OF SILICON SUBSTRATE}

\author{
AND METALLIC THIN FILM USING \\ INTERFEROMETER FOR LASER IRRADIATION \\ BELOW ABLATION THRESHOLD
}

\subsection{INTRODUCTION TO SUBMICRON AND NANO STRUCTURING OF SILICON SUBSTRATE}

Silicon micro-machining has found numerous applications in different fields. Silicon has been micro-machined for the purpose of creating optoelectronics devices, waveguides and optical telecommunication fiber gratings. An important amount of research in pulsed laser ablation and laser processing of material has been done employing pulses from nanosecond to ferntosecond time regime. For instance gratings of submicron spacing are important components in the development of wavelength specific devices, such as channel selectors and waveguide laser mirrors [52].There are number of gratings fabrication techniques that have been suggested to meet the stringent requirements. Each of these techniques interlaces some drawbacks and difficulties along with its advantages. Various grating structure of spacing larger than $0.5 \mu \mathrm{m}$ has been fabricated by holographic method, where the gratings are created by direct transfer the interference fringes from laser spot to the sample surface through laser ablation. This method had been used to create gratings on various surfaces: silica, $\mathrm{Cu}, \mathrm{Si}$, polymers, and thin metallic films 
$[53,54]$. Usually, the holography gratings techniques involve complex optical setups to achieve the common path length, $[48,54-57]$.

Graham D. Marshall [52] reported that the direct writing process (DRP) for fabricating grating structures is implemented without the need to use lithographic and/or etching techniques. The fabrication techniques can use either continuous wave $(\mathrm{CW})$ or pulsed lasers as the coherent light to create interference patterns. Some patterning techniques use (CW) lasers because its output intensity is easier to control and more commercially available. However, the drawback for using $(\mathrm{CW})$ is the difficulty in fabricating hard materials, such as silicon and glass, because numerous of the preferred holographic fabrication materials, such as dichromated gelatin and photopolymerizable compositions, are quite pragmatically fit for use with continuous wave (CW) laser sources. Using pulsed lasers for grating fabrication can reduce or solve many of the problems accompanying $(\mathrm{CW})$ laser use. Due to the different physics of Nd: YAG pulsed laser operations, a small frame pulsed laser is able to generate higher light intensity than its (CW) counterparts. For example, small frame frequency doubled Nd: YAG pulsed lasers can generate $1.1 \mu \mathrm{J}$ of energy during a $35 \mathrm{~ns}$ long pulse at $532 \mathrm{~nm}$. The advantages of Nd: YAG laser lie in three aspects having high efficiency, a long lifetime, and Polarization of the beam may be achieved simply by stressing the YAG rod, prior to frequency doubling. Nd: YAG laser is a compact solid state laser with lower operating costs than other lasers, such as $\mathrm{CO} 2$ and excimer laser. For these reasons, Nd: YAG Jaser is preferred for grating fabrication [54].

A significant amount of research in pulsed laser ablation and surface patterning of material has been done employing pulses from nanosecond to femtosecond time regime. In particular, there are several attempts of grating fabrication using different mechanisms and 
configurations are reported to date. The mechanisms responsible for material ejection and machining are strongly influenced by the physical properties of the solid, repetition rate, the laser fluence, and pulsewidth employing different materials [58-61]. T.T. Chen et al. [53] reported an interference pattern dedicated to imprinting grating on sensitive materials, such as Agfa 10E75 photo-plates, NRC H-1 photo-polymer and AZ 1350 photoresist. The laser beam was split into two beams by a broad-band 50/50 dielectric beam splitter NRC and formed an angle of approximately $18.5^{\circ}$. After that, the two split beams were brought together by using two single mirrors to interfere on the thin film surface. This setup gives a grating spacing larger than 0.5 $\mu \mathrm{m}$. The disadvantage of this grating formation method is the limiting small fixed angle which results in large grating spacing. Another disadvantage is the type of sensitive film used to fabricate grating, which is expensive and limited in use. It cannot use this setup to fabricate other materials, because the laser power and optics that are used was designed to fabricate grating on sensitive films. For glass or silicon surface require for more power and optics should be minimized to prevent energy losing. The only advantage for this setup is the simple optical parts that are used. An alternative technique is suggested to cover the drawbacks mentioned above. $\mathrm{K}$ Venkatakrishnan et al. reported and investigated planar gratings on a copper substrate using a direct writing technique [48]. This configuration reduces the number of optical components and makes the setup immune to vibration. However, the major drawback for this method is that the incident angle between two beams is just $10^{\circ}$ to $30^{\circ}$. In [57] have reported that the fabrication mechanism is completely different in the above methods. Grating fabrication uses quartz substrate coated with $100 \mathrm{~nm}$ thick copper thin film. This type of technique is fabricated by the irradiation of interference fringes of two laser beams at $400 \mathrm{~nm}$ wavelength. The reported 
method has some drawbacks, such as the limited small interfering angle $\theta$ of $5.72^{\circ}$, large grating spacing of $4 \mu \mathrm{m}$ and the alignment limitation of optical components.

The initial intent of this research will be to investigate the feasibility of using low repetition rate nanosecond laser for submicron and nanoscale material processing. The effect of pulse repetition rate and pulse energy on nanosecond laser machining of silicon and gold coated silicon wafer was studied to determine the optimum values of each of these parameters for processing $(\mathrm{C}-\mathrm{Si})$ and thin metallic film. The observations of the experimental results have been presented with an emphasis on the dependence of ablation threshold, grating spacing, ablation depth and debris on the varying of pulse energy from 0.260 to $0.125 \mu \mathrm{J}$. At each Pulse energy value, the average beam power was changed from a value slightly above the damage threshold down to the point where no machining occurred, namely below ablation threshold.

This study has been demonstrated a new technique of direct laser of surface patterning for grating fabrication in common path optical configuration. The machined structures were analyzed under scanning electron microscopy (SEM). The experimental pulse energy for this study ranged from 0.260 to $0.125 \mu \mathrm{J}$. The dependence of the ablation threshold on pulse energy and the effect of the low repetition rate and low power on the substrate have been never investigated due to the non availability of such capabilities of nanosecond laser system. The influence of the low power on ablation threshold, spacing, fluence and debris were analyzed. 


\subsection{RESULTS AND DISCUSSION}

The total average laser power at the sample surface was varied between $2.60-8.80 \mathrm{~W}$ depending on repetition rate. The laser intensity, $I$, is expressed as,

$$
I=\frac{E_{p}}{\tau_{p} A}
$$

Where, $I$, denotes the laser beam intensity, $E_{p}$, is the pulse energy, $\tau_{P}$, is nanosecond laser pulse duration and, $A$, is the spot area. The intensity of the laser beams was varied accordingly. The values are shown in Table 5-1.

\begin{tabular}{|c|c|c|c|}
\hline Power (I) & Rep. rate $(\mathbf{K H z})$ & Pulse energy $(\boldsymbol{\mu J})$ & Laser Intensity $\left(\mathbf{W c m}{ }^{-2}\right)$ \\
$\boldsymbol{P}$ & $\boldsymbol{R}_{\boldsymbol{r}}$ & $\boldsymbol{E}_{\boldsymbol{p}}$ & $\boldsymbol{I}$ \\
\hline 2.60 & 10 & 0.260 & 1.009 .316 \\
4.60 & 20 & 0.230 & 892.857 \\
5.97 & 30 & 0.199 & 772.515 \\
6.90 & 40 & 0.172 & 667.701 \\
7.60 & 50 & 0.152 & 590,061 \\
8.30 & 60 & 0.138 & 535.713 \\
8.80 & 70 & 0.125 & 485.248 \\
\hline
\end{tabular}

Table 5-1: The calculation of laser intensity for Si sample at different repetition rates

The morphology of the spacing formed was controlled by several experimental conditions but mainly by exposure time. The irradiation was for a few seconds; this will prevent thermo stress, fatigue and material hardness with the minimum of structure modifications then increase the sample specification. 
The result in Figure 5-1 shows wavy lines, because there is some tolerance in the holder moving stage, it not accurate $100 \%$ and caused the vibration and shaking the stage. Supplemental, during the machining time the stage was moving in single axis to ensure getting the interference.

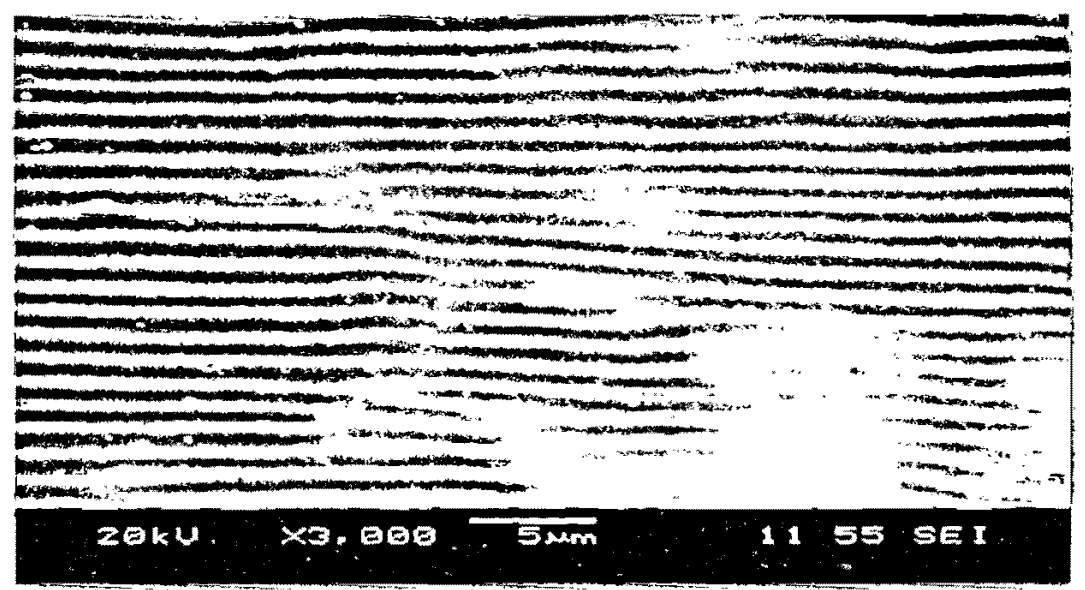

Figure 5-1: Entire spot area for Si sample at repetition rate of $10 \mathrm{KHz}$

From the Equation 1-3, the theoretical spacing is calculated as $1390 \mathrm{~nm}$. The experimentally that obtained grating spacing can been easily measured from Figure 5-2 (a) and (b). The shortest grating period (d) achieved is $1300 \mathrm{~nm}$, which is in good agreement with the theoretical calculation. To the best of our knowledge, it is the smallest grating spacing fabricated on silicon by nanosecond laser ablation at a fundamental wavelength of $1064 \mathrm{~nm}$. In addition, this setup uses common path optical configuration which gives decisive advantages when using femtosecond laser for ablation. Usually femtosecond laser setup requires an optical delay to ensure that the two pulses overlap in time and collide. The primary difficulty of direct fabrication grating by femtosecond interferometric technique is to align two femtosecond pulses travel exactly the same optical path. Since laser beam travels at the light speed, a small deviation in the 
optical path will lead to poor tempo overlap of the two pulses. For femtosecond laser interferometer, the presented common path configuration facilitates not only interference but also the ease of alignment.

The results that represented by Figure 5-2 ( $a, b$, and c) shows influence of pulse energy and repetition rate on grating writing. It found that at higher repetition rate efficiently offer sharp edge and better grating quality. General looking to the results at 30,20 and $10 \mathrm{KHz}$ reveals that at higher repetition rate the energy will be low and the energy level will be closed to threshold and result in low crust layer for better gratings. The proper amount of energy signifies there is enough energy per pulse to start machining and the average threshold is fairly well enough to start machining with best equality. Good grating comprise the edge quality of grating spacing is acute sharp and pointed with high aspect ratio, as appeared in Figure 5-3 (a, b, c, and d).

It can be seen from Figures 5-4 and 5-5 that repetition rates, pulse energy and dwell time has the major effects on the quality and profile of the grating pattern. The results demonstrate the effect of dwell time variation. More debris is observed as seen in Figures 5-4-c and 5-5-c, which describes longer dwell time, can result for extremely debris, molten material and very low structure quality. The molten debris in consequence of long time of $200 \mathrm{~ms}$ totally gives unclear pattern that demolish the grating shape. Figures 5-4-a and 5-5-a have been exhibited that grating fabrication with nanosecond laser at $100 \mathrm{~ms}$ can produce a very high quality with least of debris. The quality of the pattern obtained and the amount of debris seen decrease at a shorter time and higher machining speed. This will lead to reduction in surface debris, increase in sample strength and less contamination. 


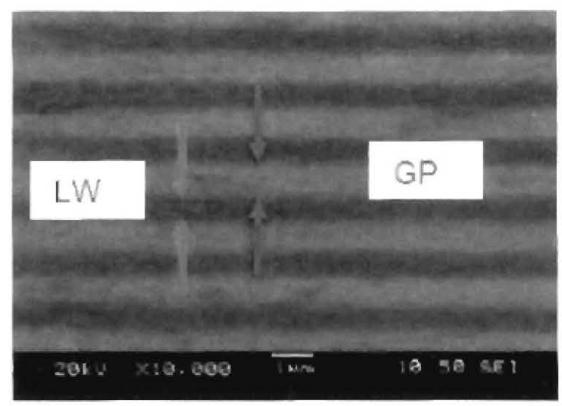

(a)

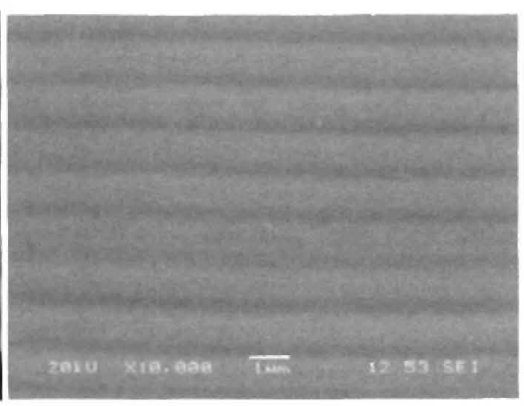

(b)

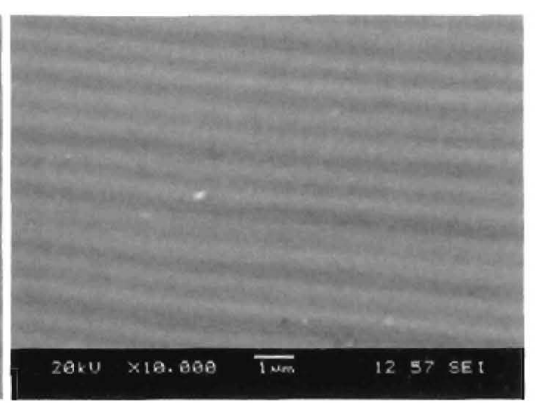

(c)

Figure 5-2: Si samples at different repetition rate, (a) repetition rate of $30 \mathrm{KHz}$, pulse energy of 0.199 $\mu \mathrm{J}$ shows line width ( $\mathrm{LW}$ ) of $460 \mathrm{~nm}$ and grating period (GP) of $1300 \mathrm{~nm}$, (b) $20 \mathrm{KHz}$, pulse energy of $0.230 \mu \mathrm{J}$, and (c) $10 \mathrm{KHz}$. high pulse energy of $0.260 \mu \mathrm{J}$.

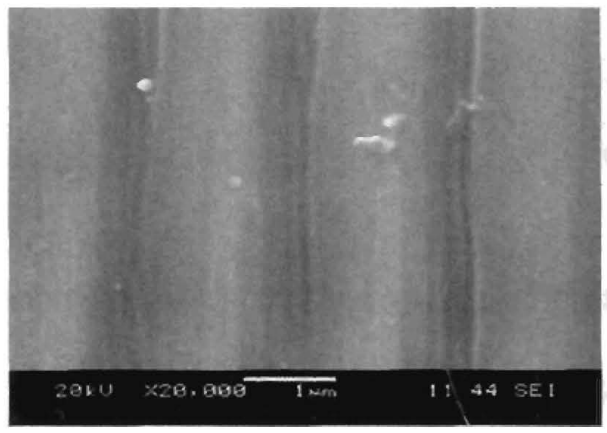

(a)

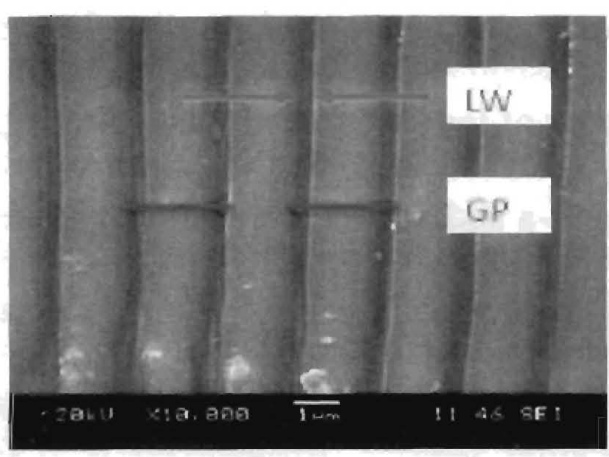

(c)

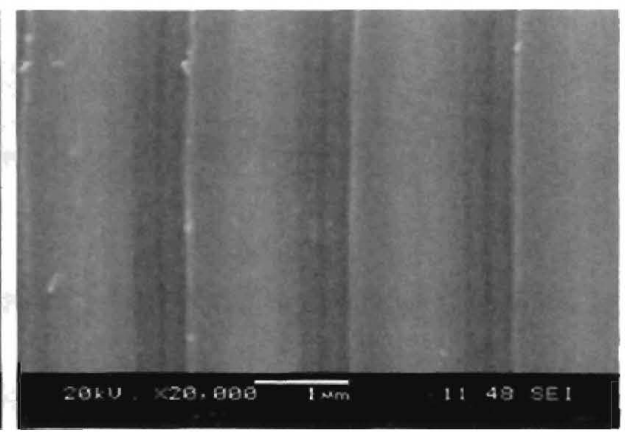

(b)

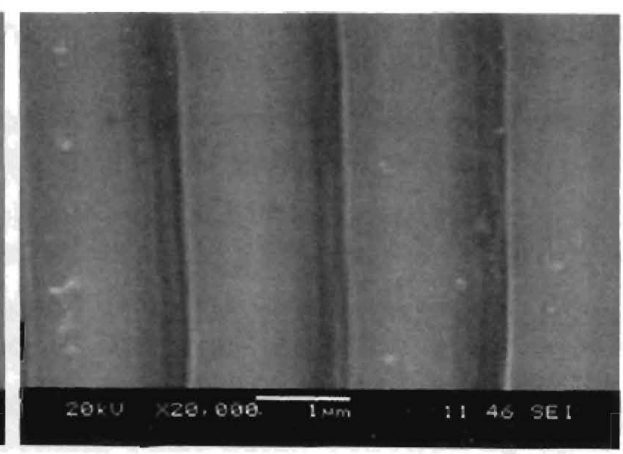

(d)

Figure 5-3: Si samples at different rep. rate, (a) at $40 \mathrm{KHz}$, pulse energy of $0.172 \mu \mathrm{J}$, (b) $50 \mathrm{KHz}$, pulse energy of $0.152 \mu \mathrm{J}$, (c) $60 \mathrm{KHz}$, pulse energy of $0.138 \mu \mathrm{J}$ exhibits $(L W)$ of $320 \mathrm{~nm}$ and $(G P)$ of $1300 \mathrm{~nm}$ , and (d) $70 \mathrm{KHz}$, pulse energy of $0.125 \mu \mathrm{J}$ 


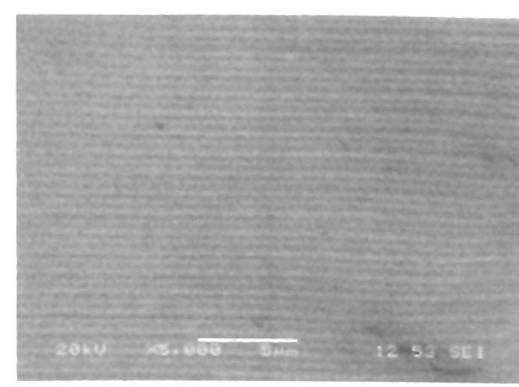

(a)

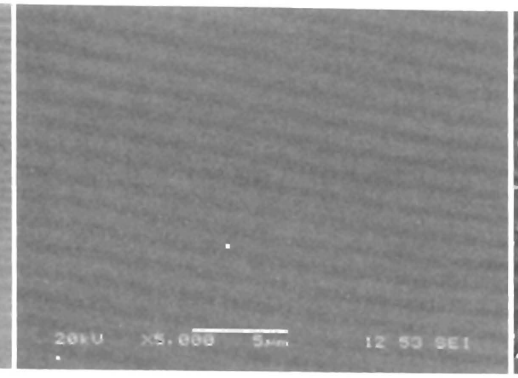

(b)

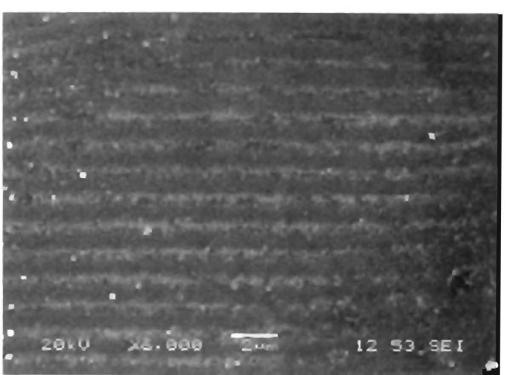

(c)

Figure 5-4: At repetition rate of $10 \mathrm{KHz}$ exhibit three images of different quality at dwell time of (a) $100 \mathrm{~ms}$ (b) $150 \mathrm{~ms}$ (c) $200 \mathrm{~ms}$.

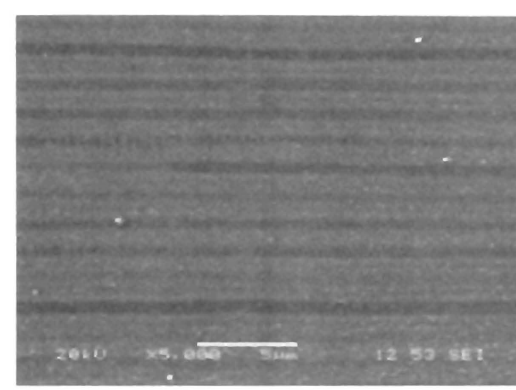

(a)

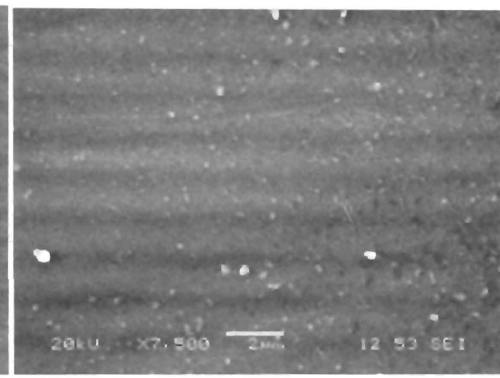

(b)

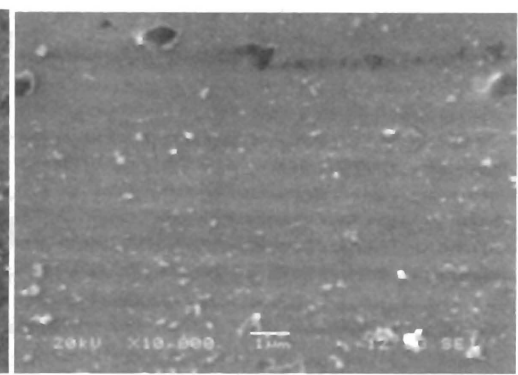

(c)

Figure 5-5: For different dwell time of 100, 150 and $200 \mathrm{~ms}$ at repetition rate of $20 \mathrm{KHz}$ shows three results of different quality.

\subsection{PRACTICAL CUNSIDERATION}

\subsubsection{POSSESSING PARAMETERS}

\subsubsection{Effect of Pulse Energy}

The pulse energy $E_{p}$ of two interfering beams is set to be equal to each other and calculated from the Equation 5-2. 


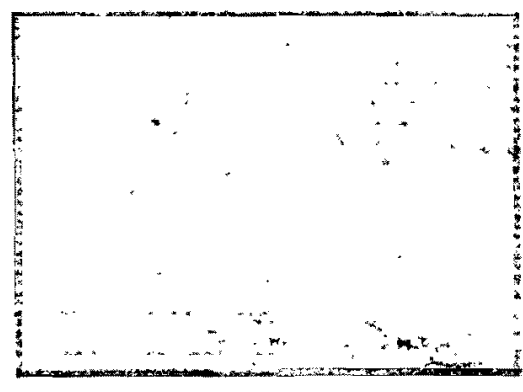

(a)

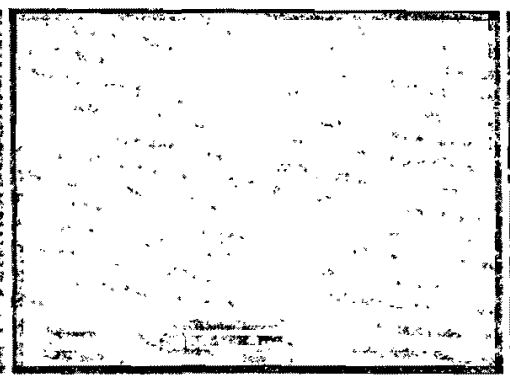

(b)

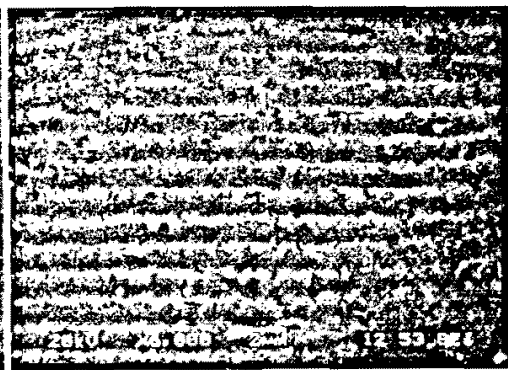

(c)

Figure 5-4: At repetition rate of $10 \mathrm{KHz}$ exhibit three images of different quality at dwell time of (a)

$$
100 \mathrm{~ms} \text { (b) } 150 \mathrm{~ms} \text { (c) } 200 \mathrm{~ms} \text {. }
$$

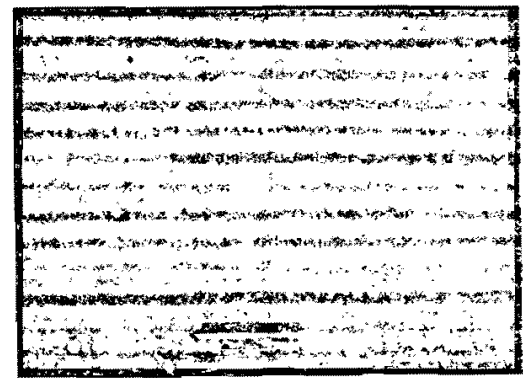

(a)

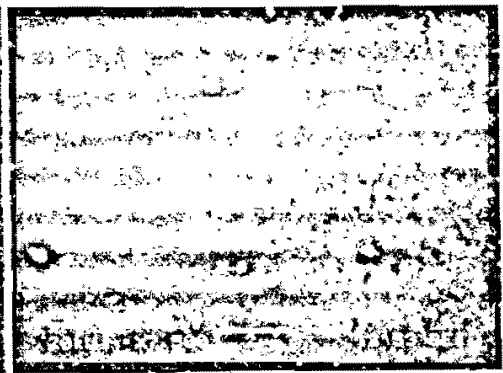

(b)

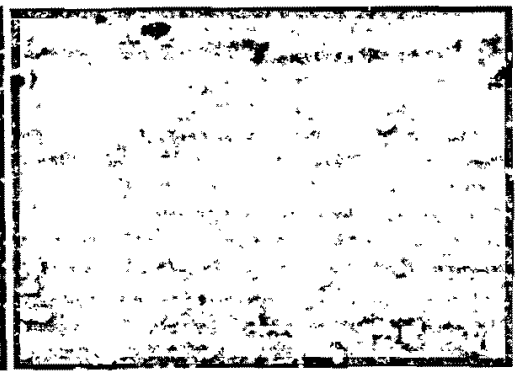

(c)

Figure 5-5: For different dwell time of 100,150 and $200 \mathrm{~ms}$ at repetition rate of $20 \mathrm{KHz}$ shows three results of different quality.

\subsection{PRACTICAL CONSIDERATION}

\subsubsection{POSSESSING PARAMETERS}

\subsubsection{Effect of Pulse Energy}

The pulse energy $E_{p}$ of two interfering beams is set to be equal to each other and calculated from the Equation 5-2, 


$$
E_{p}=\frac{P}{R_{r}}
$$

Where, $P$, denotes the laser beam power, $R_{r}$, denotes the laser repetition rate.

For example, by variation and changing the repetition rates $(30,20$ and $10 \mathrm{KHz})$, the pulse energy was $0.199,0.230$ and $0.260 \mu \mathrm{J}$, respectively. The results of this investigation are in agreement with results found by many authors [62-64] who showed that the properties of $\mathrm{Si}$ is greatly influenced by the characteristic of structure produced by laser ablation near or under the threshold ablation $\emptyset_{\mathrm{th}}$. The morphology of a nanosecond laser induced surface structure depends on both pulse energy and time duration for laser irradiation. The evolution of semiconductor surface (including silicon) morphology has resulted from the irradiation of two interfering beams of $1064 \mathrm{~nm}$ wavelength. Figure 5-6 shows the dependence of pulse energy on beam threshold power which acquired for both theoretical and experimental data.

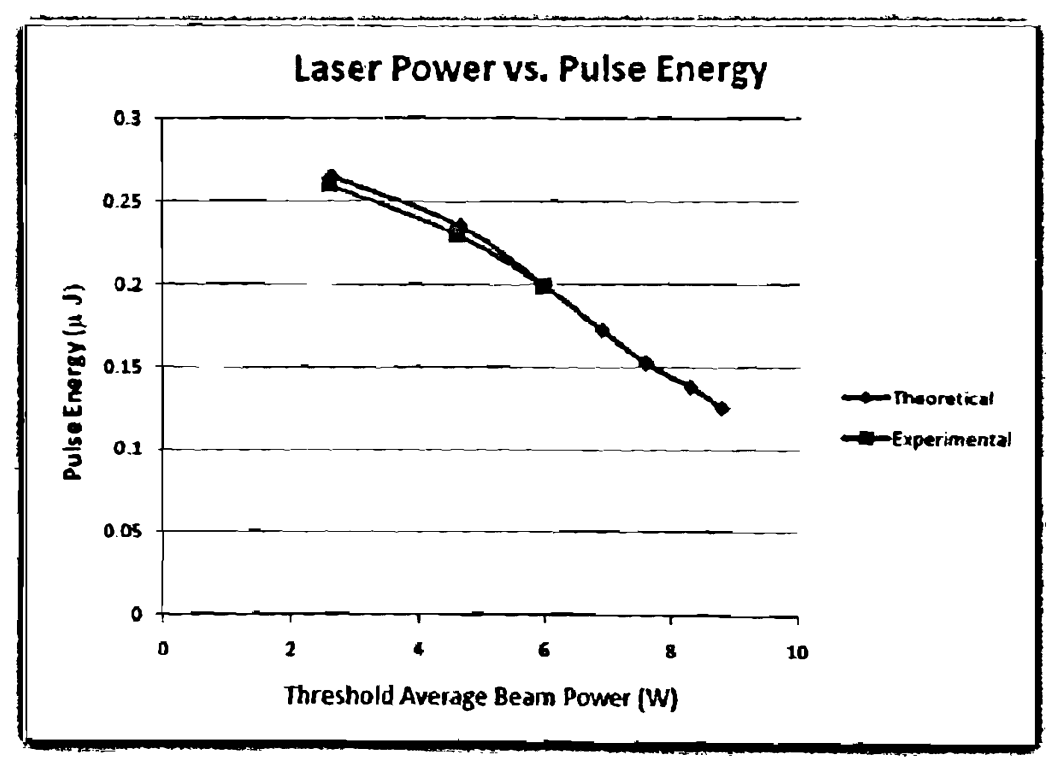

Figure 5-6: Threshold average beam power vs. pulse energy for low repetition rate of $10 \mathrm{KHz}$ to 70 $K \mathrm{~Hz}$ 


\subsubsection{Effect of Repetition Rate}

The experimental result shown that the shape and quality of the grating have been varies with the repetition rate. As shown in Figure 5-3 (a, b, c, and d), it can be seen that the structure boundaries to be much sharper and with good grating edge acuity at higher repetition rates. Thorough study for the grating parameter will provides a beneficial indicator for line width (LW) shape, quality and size under different repetition rate. As shown in Figure 5-7 the line width is inverses proportional with the influence of repetition rate. At $30 \mathrm{KHz}$ the line shape was more homogenous and has sharp edges while at $10 \mathrm{KHz}$ the shape and size of line width was much bigger and not upright. By another meaning it's possible to consider the line width variation as a function of laser frequency.

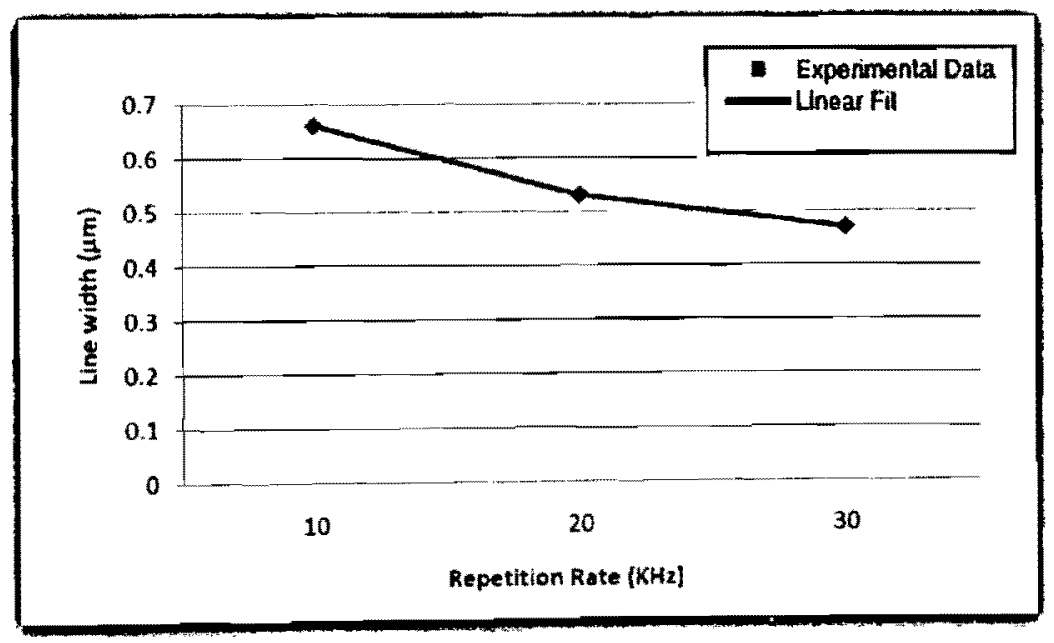

Figure 5-7: Shows the grating feature quality through effect of repetition rate on line width

The dependence of laser power and repetition rate explained below by Figure 5-8, which display the major effect of laser frequency on laser threshold power. As seen in Figure 5-8, graph demonstrating threshold average beam power for different laser repetition rates and the effect of 
pulse repetition rate on beam power. However, the higher the laser repetition rate is, the higher the threshold average beam power becomes. It is important to note that flats out in the range of repetition rate between $10-100 \mathrm{KHz}$, will remains at constant threshold fluence. This is due to the fact that minimum threshold fluence is required to start the ablation process. If the laser fluence goes below this minimum threshold fluence of $10 \mathrm{KHz}$, no ablation occurs no matter how much machining time is fed to the target surface. Therefore, as Figure 5-8 demonstrates, as long as the threshold fluence remains almost constant, increase in the pulse repetition rate leads to the increase in threshold laser power.

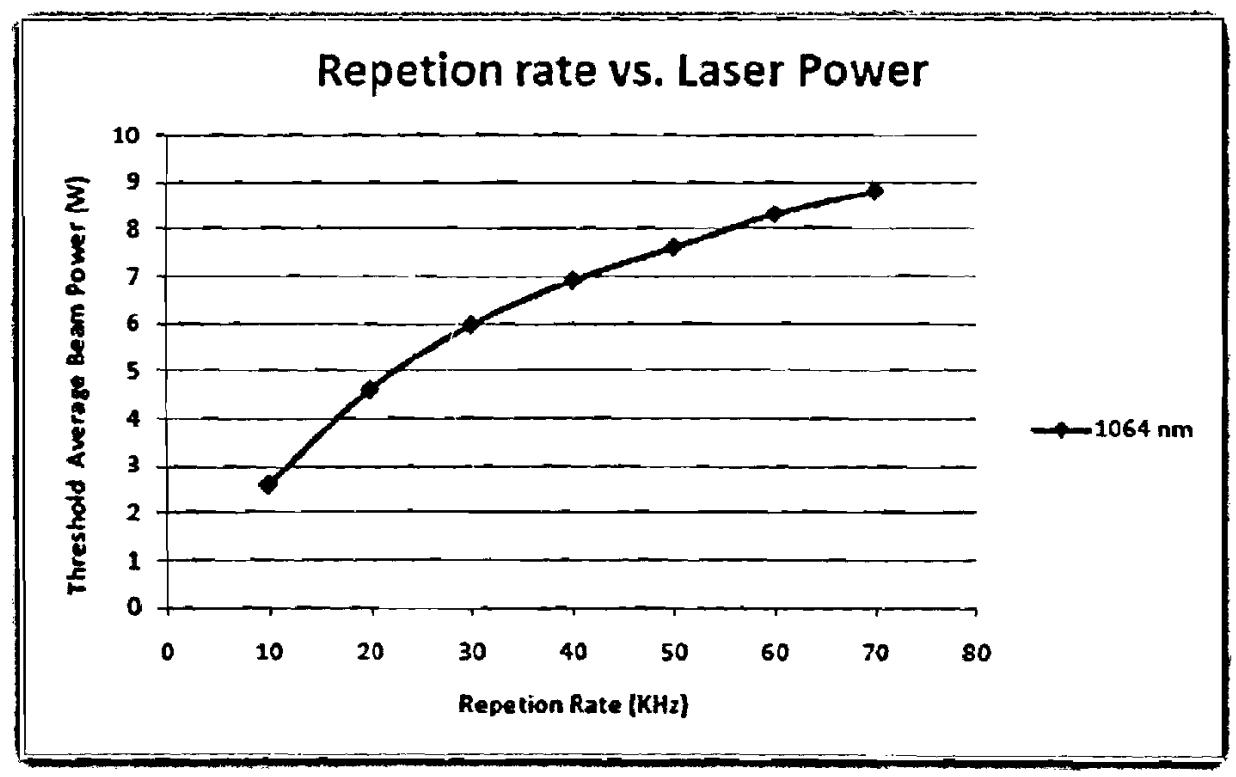

Figure 5-8: Laser repetition rate vs. threshold average beam power

\subsubsection{Effect of Laser Intensity}

The morphology of nanosecond laser induced surface structure depends on both pulse energy, which represented by laser intensity and time duration for a laser shot. The evolution of semiconductor surface (including Silicon) and metallic thin film (include Gold) morphology has 
resulted from irradiation of two interfering beams of $1064 \mathrm{~nm}$ wavelength. The dependence of intensity on pulse energy can derive by the Equation 5-1.

For a thin metallic film (Including gold), the ablation with lasers in the nanosecond region at medium and high laser fluence maybe proceeds and take place via electron-ion recombination or ionization processes. This phenomenon is not observed in the femtosecond regime yet, for nanosecond regime, Figure 5-9 shows experimental result for thin gold film sample at low repetition rate of $10 \mathrm{KHz}$ and pulse energy $E_{\mathrm{p}}$ was $0.260 \mu \mathrm{J}$. The incoming laser was absorbed by the electrons and the energy was passed to the metal lattice by electron-photons interplay. Due to the fast energy that transferred from electrons to the lattice, increasing of the lattice temperature and caused melting then evaporate of gold coating. At later time rapidly the cooling mechanism begin which take place between two pulses period, the electrons and thin gold film lattice afterward immediately cooled off with energy transfer off the lattice. Rapid under-cooling was resulted in fast solidification and re-crystallization. Such this is mechanism phenomenon resulting in nano bump gratings formation $[65,66]$. The threshold laser fluence which is necessary for evaporation with long laser pulses grows as $\tau_{\mathrm{L}}{ }^{1 / 2}$. In case of ablation with long laser pulses there is enough time for the thermal wave to propagate into the target and to create a relatively large layer of melted material [7]. In our application, it is obligatory to consider the target surface temperature for the case of succession of laser pulses rather than only one pulse. The properties of $\mathrm{Au}$ are greatly influenced by the characteristic of nanostructure fabricated by laser ablation near or below threshold ablation. 


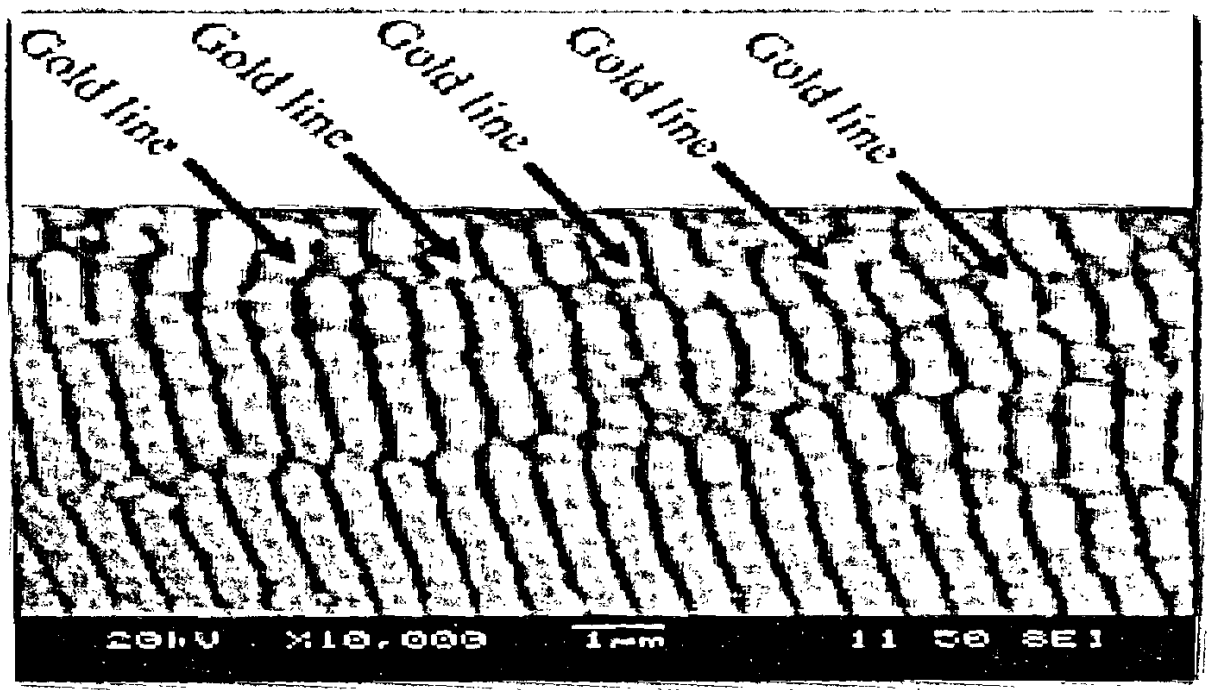

Figure 5-9: SEM image of surface of sample with Au thin film after irradiation of low repetition rate of $10 \mathrm{KHz}$ and high pulse energy

As mentions by [7], If can assume that the thermalization within the electron subsystem is very fast and that the electron and the lattice subsystems (phonon component) can be characterized by their temperatures $\left(\mathrm{T}_{\mathrm{e}}\right.$ and $\left.\mathrm{T}_{i}\right)$, It can further be assumed that the laser energy will be absorbed in a layer much thinner than the penetration depth of the heat wave. Therefore the energy transport into the metal can be described by the following one-dimensional, twotemperature diffusion model,

$$
\begin{aligned}
& \mathrm{C}_{\mathrm{e}} \frac{\partial \mathrm{T}_{\mathrm{e}}}{\partial \mathrm{t}}=-\frac{\partial \mathrm{Q}(\mathrm{z})}{\partial \mathrm{z}}-\gamma\left(\mathrm{T}_{\mathrm{e}}-\mathrm{T}_{\mathrm{i}}\right)+\mathrm{S} \\
& \mathrm{C}_{\mathrm{i}} \frac{\partial \mathrm{T}_{\mathrm{i}}}{\partial \mathrm{t}}=\gamma\left(\mathrm{T}_{\mathrm{e}}-\mathrm{T}_{\mathrm{i}}\right) \\
& \mathrm{Q}(\mathrm{z})=-\mathrm{k}_{\mathrm{e}} \frac{\partial \mathrm{T}_{\mathrm{e}}}{\partial \mathrm{z}}, \quad \mathrm{S}=\mathrm{I}(\mathrm{t}) \mathrm{a} \alpha \exp (-\alpha \mathrm{z})
\end{aligned}
$$


Where, $z$, is the direction perpendicular to the target surface, $Q(z)$, is the heat flux, $S$, is the laser heating source term, $I(t)$, is the laser intensity, $a$, is the laser absorption, $\alpha$, are the surface transmissivity and the material absorption coefficient, $C_{e}$, and , $C_{i}$, are the heat capacities (per unit volume) of the electron and lattice subsystems, $\gamma$, is the parameter characterizing the electron-lattice coupling, and, $k_{e}$, is the electron thermal conductivity.

In the above Equations a thermal conductivity in the lattice subsystem (phonon component) is neglected. The electronic heat capacity is much less than the lattice heat capacity; therefore electrons can be heated to very high transient temperatures. When the electron temperature (in units of energy) remains smaller than the Fermi energy, the electron heat capacity and the non-equilibrium electron thermal conductivity are given by Equation 5-6,

$\mathrm{C}_{\mathrm{e}}=\mathrm{C} \square_{\mathbf{e}} \mathrm{T}_{\mathrm{e}}$, Eq 5-6

Where, $\mathrm{C}_{\mathrm{e}}$, is a constant, Electron thermal conductivity, $\mathrm{k}_{\mathrm{e}}$, is given by Equation 5-7,

$$
\mathrm{K}_{\mathrm{e}}=\mathrm{k}_{\mathrm{o}}\left(\mathrm{T}_{\mathrm{i}}\right)
$$

Here, $\mathrm{k}_{0}$, is the conventional equilibrium thermal conductivity of a metal [36].

Equations 5-3, 5-4 and 5-5 have three characteristic time scales $\tau_{e}, \tau_{i}$ and $\tau_{L}$, where $\tau_{e}=$ $\mathrm{C}_{\mathrm{e}} \gamma \gamma$ is the electron cooling time, $\tau_{\mathrm{i}}=\mathrm{C}_{\mathrm{i}} / \gamma$ is the lattice heating time $\left(\tau_{\mathrm{e}}<\tau_{\mathrm{i}}\right)$ and $\tau_{\mathrm{p}}$ is the duration of the laser pulse. These parameters define the regime of the laser-metal interaction in nanosecond regime. 
In our application, for ablation with nanosecond laser pulses, when the condition, $\tau_{\mathrm{P}} \gg$ $\tau_{i}$, is fulfilled. In this case the electron and lattice temperatures are equal $T_{e}=T_{i}=T$, also (Equations 5-3, 5-4, and 5-5) reduces to Equation 5-8,

$$
C_{i} \frac{\partial T}{\partial t}=\frac{\partial}{\partial z}\left(k_{0} \frac{\partial T}{\partial z}\right)+I_{a} \alpha \exp (-\alpha z)
$$

Axiomatic, metals need much more energy to vaporize than to melt. During the interaction the main source of energy losses is the heat conduction into the solid target. The heat penetration depth is given byl $\sim(H D t)^{1 / 2}$, where $\mathrm{HD}$ is the heat diffusion coefficient, $H D=\mathrm{k}_{0} /$ $\mathrm{C}_{\mathrm{i}}$. Note that for long-pulse laser ablation of metal targets the condition, $\operatorname{HD} \tau_{\mathrm{p}} \alpha^{2} \gg 1$, is usually fulfilled. The energy deposited inside the target per unit mass is given by $E_{m} \sim I_{a} t / \rho l$ where $\rho$ is the density. When at a certain moment $t=t_{t h}$ this energy becomes larger than the specific heat of evaporation $\Omega$, significant evaporation occurs. From the condition $E_{m} \sim \Omega$ get $t_{\mathrm{t}} \sim H D(\Omega \rho / I)^{2}$ was acquired. Thus, the condition for strong evaporation, $E_{m}>\Omega$ (or $\tau_{P}>t_{t h}$ ), for laser intensity and fluence can be written as,

$$
\begin{aligned}
& I>\mathrm{I}_{\mathrm{th}} \sim \frac{\rho \Omega(H D)^{1 / 2}}{\tau_{\mathrm{P}}^{1 / 2}} \\
& \mathrm{~F}>\mathrm{F}_{\mathrm{th}} \sim \rho \Omega(\mathrm{HD})^{1 / 2} \times \tau_{\mathrm{P}}^{1 / 2}
\end{aligned}
$$

Where, $\rho$, is the density and, $\Omega$, is the specific heat of evaporation (per unit mass), and these two Equations 5-9 and 5-10 is for laser intensity and fluence respectively. Below Table 5-2 that shows the thermo-physical and optical properties of gold which used in the calculation of heat conductions through pulses laser ablation. 


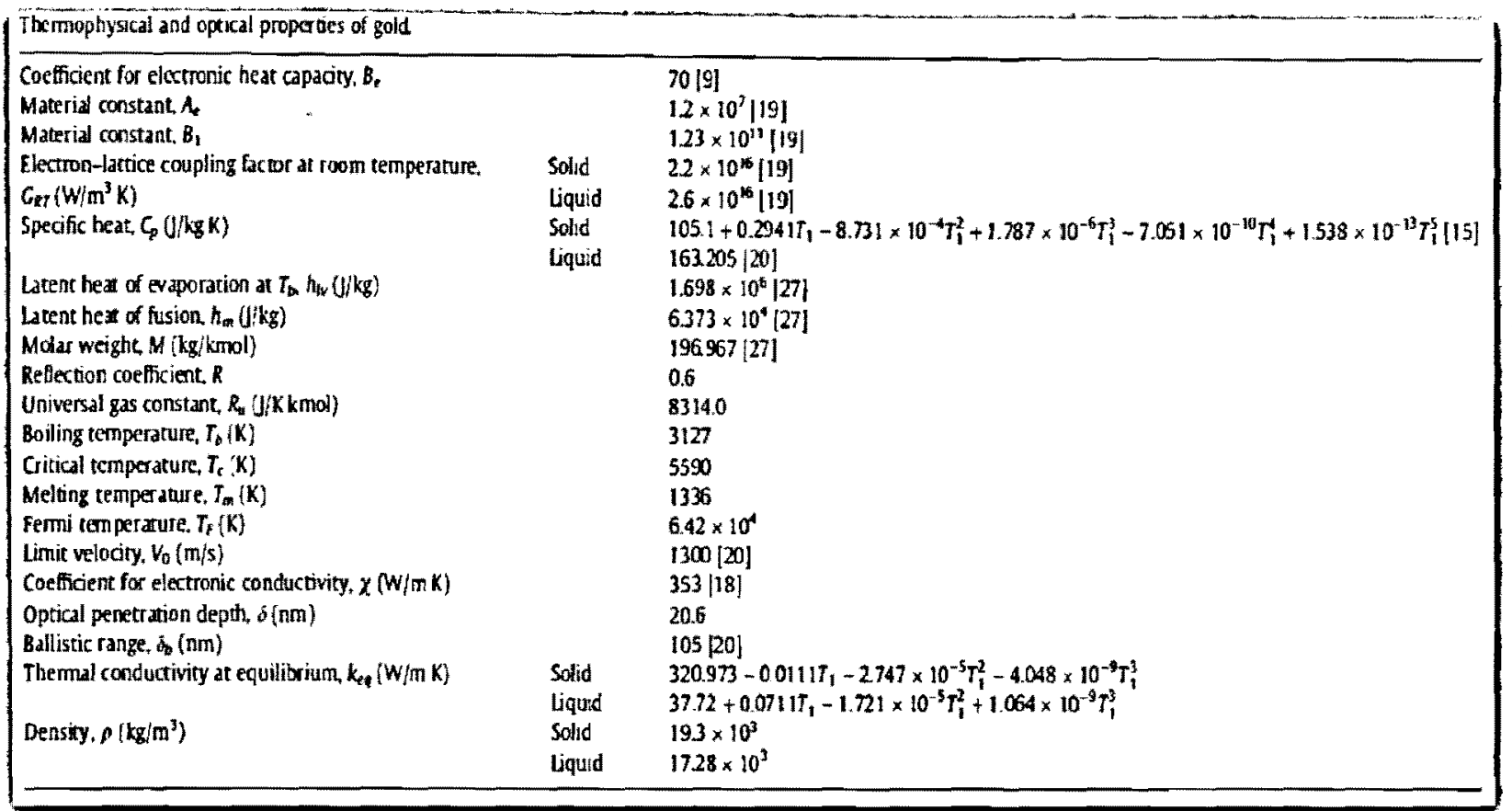

Table 5-2: Thermo-physical and optical properties of A u [67]

\section{$5.4 \quad$ SUMMARY}

In conclusion, surface grating on a silicon sample and thin gold film have been fabricated. The competitive edge is using nanosecond lasers as a tool and the use of a common path optical configuration for automatic interference. At the fundamental wavelength of 1064 $\mathrm{nm}$, grating spacing of $1300 \mathrm{~nm}$ was fabricated. Smaller grating can be expected with shorter wavelength of $2 \mathrm{nd}, 3 \mathrm{rd}$ and 4 th harmonic generation of fundamental wavelength. In summary, the grating shape is distinguishable and sharper at higher repetition rates. Some advantages of this technique include that the quality is very uniform, machining time is very short, ability to reduce the fringe space to very fine features, simplifying the optical setup, low in cost, self alignment, equal beam intensity, and also immunizing the system to extraneous_and inherent vibrations. Future work can comprise of increase in the frequency of the gratings and reduction of the feature sizes. 


\section{CHAPTER 6 \\ NANOSECOND LASER FOR SILICON NANO}

PATTERNING

\subsection{INTRODUCTION}

Nano patterns fabrication have attracted attention in industrial applications. As electronic components become more compact, the miniaturization of these devices requires a smaller feature size, much more density per area and fast patterning technique with large-scale. Although much research has been carried out using femtosecond laser into industrial application, nanosecond laser still remain able to achieve surface patterning as cheaper and reliable laser resource. To demonstrate the advantages of using low repetition rate nanosecond laser system, feature size and spacing using different laser parameters like pulse repetition rate and pulse energy are presented for silicon samples. However, a systematic investigation of further relevant parameters enables the processing quality and size to be optimized.

A significant amount of research in pulsed laser structuring and laser processing of material in field of surface patterning has been done employing pulses from nanosecond to femtosecond time regime. Furthermore fabrication of micro and nanodots structures and maskless surface patterning of various materials is one of the most important applications. Dots patterns have great interest for fundamental techniques in the development of data storage applications as well as attracting interest in increasing the densities of integrated circuits, quantum devices, and recording media. However, the fabrication of such nanodots has been a major challenge. Nanodots fabrication techniques have been suggested to meet the severe 
requirements of generate highly efficient dots arrays by disposing dots density, dots shape, dots pitch and arrays spacing. Fabrication methods at micro and nanometer scale are developing more important from the industrial applications viewpoint. These technologies have used to fabricate dots and patterns at micro and nano scales on Silicon and thin metallic films deposited on silicon substrate. Laser ablation therefore is preferred technique for dots arrays over the large-scale machining area by a single shot, fast, clean operating and economic.

As in [68], has been investigated that periodic structures of submicron dots were fabricated by an Nd: YAG second harmonic linearly polarized laser of $532 \mathrm{~nm}$ with repetition frequency of $10 \mathrm{~Hz}$ and pulse width of $6 \mathrm{~ns}$ to Silicon and Nickel thin films deposited on $\mathrm{SiO} 2$ film. The interference between the laser beam and the scattered surface wave leads to the spatial periodicity of beam energy density distribution on the samples surface. Other group has demonstrated the bump formation on a glass surface with a poly-methyl methacrylate (PMMA) film coating using a focused femtosecond laser pulse [69]. The bumps were formed as a result of suppressing material emission from the glass surface by a shielding effect of plasma generated through ablation of the (PMMA) film and by physical blocking of the (PMMA) film using a focused femtosecond laser pulse. The thin transparent coating was formed of (PMMA). A thinner film with a thickness of $0.7 \mu \mathrm{m}$ produced a reduced shielding effect, forming an exploded bump with a small pit at its center and debris around the perimeter. D. Mills et al [70] was investigated that at enough low laser fluence, frequent arrays of Si microdots are formed by irradiating a Si surface that was covered with a $\mathrm{Cr}$ thin film grating. Incident non polarized laser pulses in air at $0.4 \mathrm{~J} \mathrm{~cm}^{-2}$ of 1000 laser shots leads to the ablation of silicon that formed of a 140 $\mathrm{nm}$ pitch $\mathrm{Cr}$ grating. The result was regular bright balls like silicon dioxide and no holes are observed. It is likely that the structures grow due to molten silicon migrating from the hotter 
regions of the surface to the cooler areas and solidifying formation. This group was mentioned in their investigation that in place of a frequent array of Si microdots of $1 \mu \mathrm{m}$ diameter, $1 \mu \mathrm{m}$ microdot spacing and $3 \mu \mathrm{m}$ row spacing is created directed like parallel bands in the direction of the ablated chromium strips on clean silicon wafer under low power irradiation. In [71] the group has reported that the periodic structure formation of two-dimensional was fabricated on the surface of silica glass by a single shot of three interfered femtosecond laser pulses. Microstructures including both micro holes and micro orbicular platform have been investigated due to the interaction between the Silica glass and the femtosecond laser at a wavelength of 800 $\mathrm{nm}$. The fabricated microstructures can be ascribing the formation of the plasma and the molten liquid on the surface of the bulk silica glass at different energy level of the pulse. Several groups $[72,73]$ have prospered for fabrication of the periodic structures in photosensitive transparent materials by interfere multi beams of femtosecond laser pulses with the assist of the diffractive beam splitter. The limitation of this method represents by the quantity of pulses energy and the angles which bounded by two beams. Therefore this will result the difficulty of microstructure fabrication with small period, also for the materials that has big energy band gap such as silica glass is difficult of fabrication. These techniques mingle advantages with some drawbacks. Each of these techniques is preferred due; their advantages include such, easy fabrication process, low cost and fast.

In previous Chapters, laser machining processing combined many material changes and stages have been mentioned. As [10] has reported, amorphization, melting, re-crystallization, nucleated vaporization and ablation occur as part of the laser machining of silicon wafers. Features such as dots as well as ripples and columns are created during these stages. The resolidification happens in two stages; amorphization and re-crystallization. The main difference 
between these two processes rests with amount of energy deposited in the material in temperature form and the following cooling velocity. At lower temperatures, characterized by short irradiation periods, the material does not re-crystallize after melting and remains in an amorphous state. In any event, if the temperature is high quite, then the slow cooling will permit re-crystallization. From the other point of view, the modification threshold refers to the laser fluence required to initiate the amorphization process, namely changing the silicon surface from solid to liquid. After melting, re-crystallization of the liquid silicon layer occurs [74]. Certainly, if the laser fluence is above the ablation threshold, the silicon wafer is machined. In our experiments, a new technique of submicron dots arrays fabricating by direct laser irradiation below threshold using a self-aligned common path optical configuration, the dots formed at very low fixed laser fluence of $0.129 \mathrm{~J} / \mathrm{cm}^{2}$.

In our experimental results, the Figures clearly show that two intersection lines have been formed at same time, first related to gratings formula and the other as ripples patterning structure related to theory of interfere the incident light with the surface wave. Sub-micron dots structure has been fabricated by direct fabrication through transfer the interference fringes from a laser spot to the sample surface.

\subsection{RESULTS AND DISCUSSION}

\subsubsection{SUBMICRON DOTS ARRAYS}

The existent surface structure change of submicron dots induced by $1064 \mathrm{~nm}$ laser is presented in Figure 6-1 and Figure 6-2 as a function of laser energy density, wavelength, and pulse duration as well as fluence intensity. Laser fluence intensity is $0.129 \mathrm{Jcm}^{-2}$ and was calculated from the ratio of the pulse energy that attains the value needed for silicon melting 
against spot area. These dot structures were defined by two kinds of mechanism formation which performed approximately at same time. Furthermore, the dots arrays was governed and arose from intersect two patterns, first is grating that generated by direct interference writing formula and second is ripple that generated by interference between the incident laser and the frozen capillary waves (FCW). The continuous lines in X-axis were grating periods (d) induced by the interferometer technique and the discontinuous line breakage in the $\mathrm{Y}$-axis is a result of the (FCW) grating.

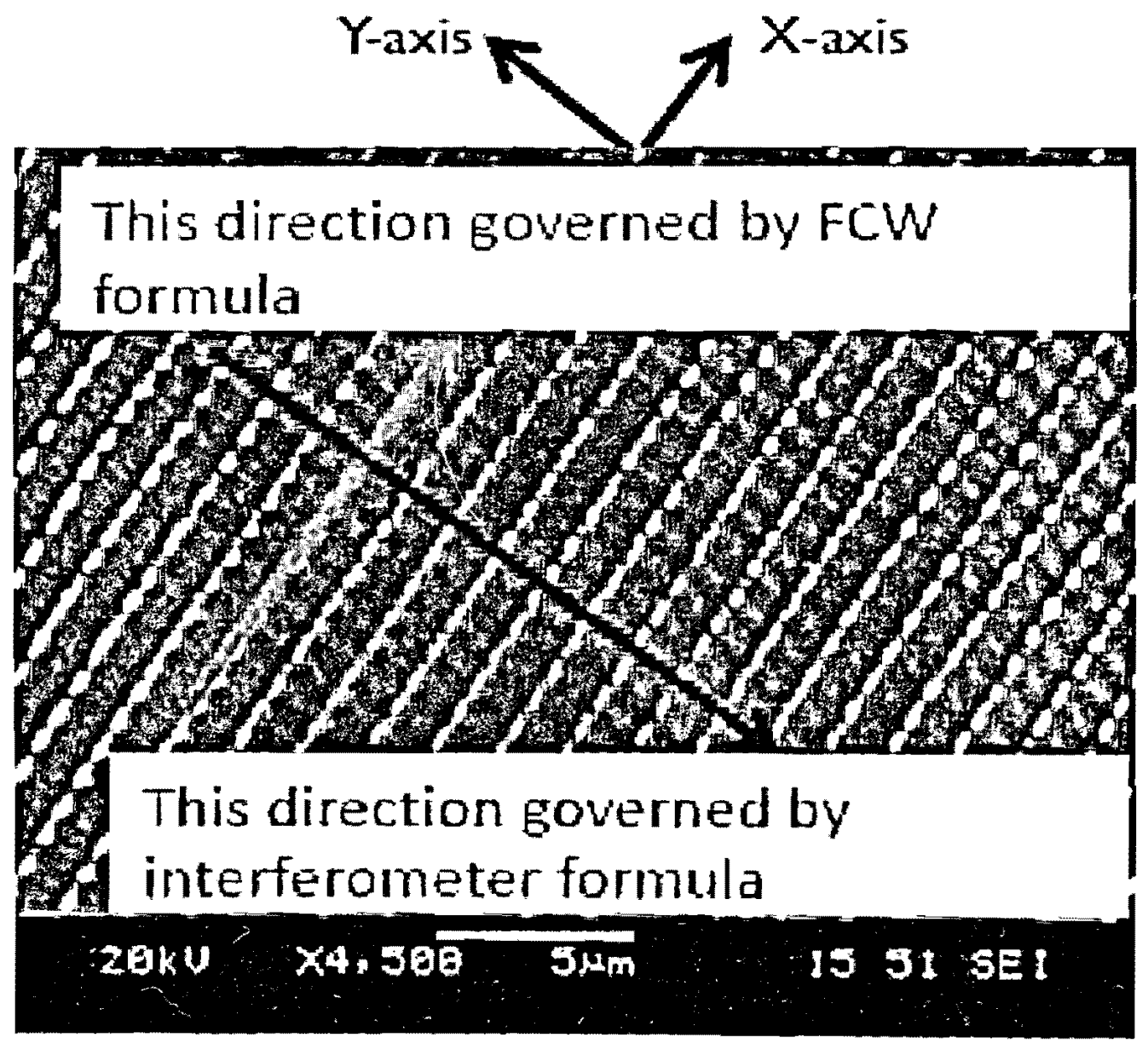

Figure 6-1: Submicron dots structure at repetition rate of $10 \mathrm{KHz}$ and pulse energy of $0.29 \mu \mathrm{J}$. 
against spot area. These dot structures were defined by two kinds of mechanism fonnatson which performed approximately at same time. Furthermore, the dots arrays was governed and arose from intersuct two patterns. first is grating that generated by direct interference wrsting formula and second is ripple that generated by interference berweon the incident laser and the frozen capillary waves (FCW). The contunuous lines in X-axis were grating periods (d) induced by the interferometer technique and the discontinuous line breakage in the Y-axis is a result of the (FCW) grating

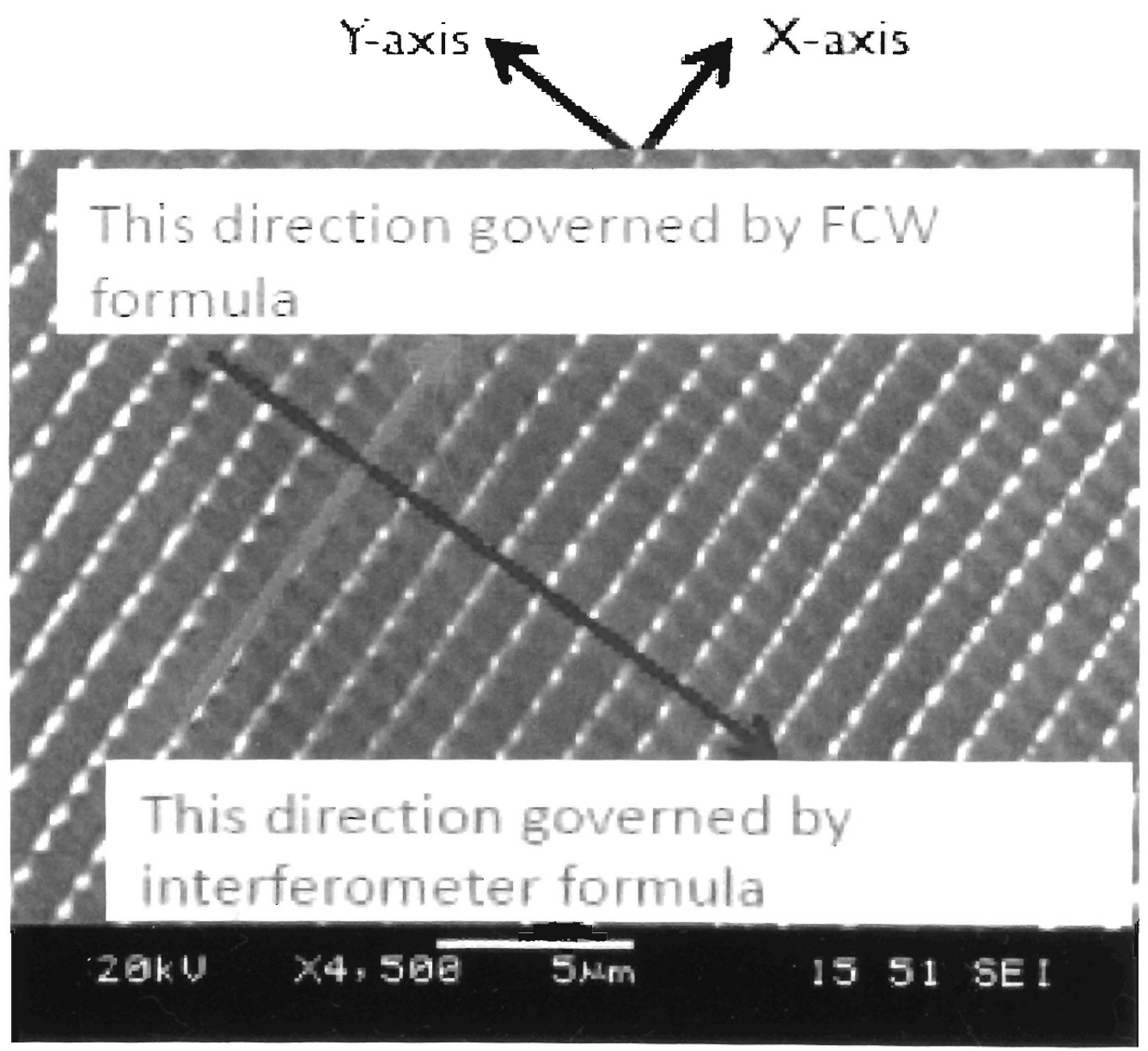

Figure 6-1: Submicron dots structure at repetition rate of $10 \mathrm{KHz}$ and pulse energy of $0.29 \mu \mathrm{J}$. 


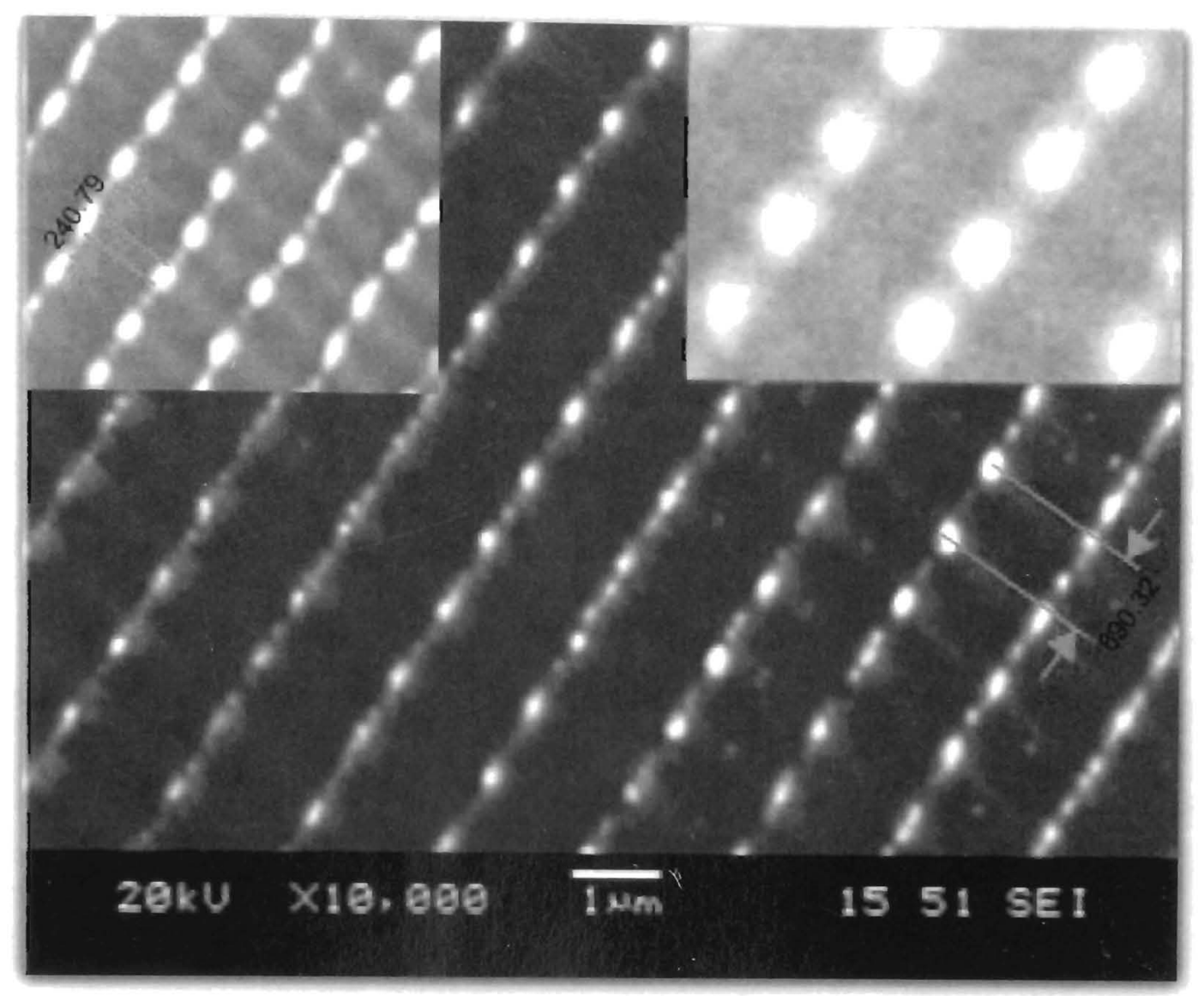

Figure 6-2: Zoom-in of submicron dots array of multi sceneries at repetition rate of $10 \mathrm{KHz}$ and pulse energy of $0.29 \mu \mathrm{J}$.

The forming of a submicron dots pattern on Si was such as regular arrays with average inıcrodots size of $240 \mathrm{~nm}$ and dots spacing was of $890 \mathrm{~nm}$, in the average. The dots structure was shaped at the interscetion point of the interferometer and the (FCW) grating. While the dimensions ut an array duc laser structuring depend upon the wavelength, and the spacing between two adjacent elements which is usually close to laser wavelength. Experimentally the average period $(d)$ of the interferometer grating was $1388 \mathrm{~nm}$, which is in agreement with results reported in Chapter 5. 


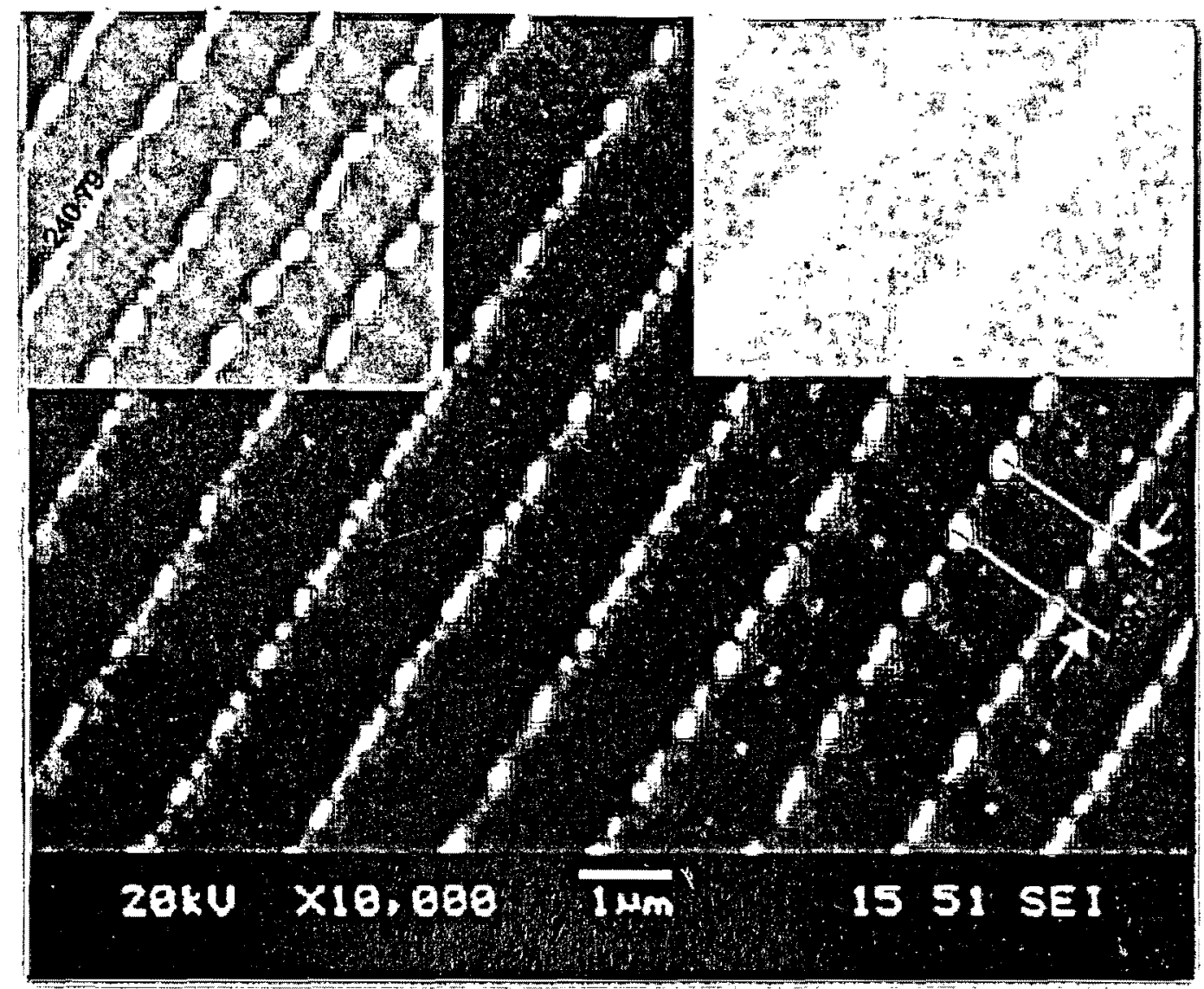

Figure 6-2: Zoom-in of submicron dots array of multi sceneries at repetition rate of $10 \mathrm{KHz}$ and pulse energy of $0.29 \mu J$.

The forming of a submicron dots pattern on Si was such as regular arrays with average microdots size of $240 \mathrm{~nm}$ and dots spacing was of $890 \mathrm{~nm}$, in the average. The dots structure was shaped at the intersection point of the interferometer and the (FCW) grating. While the dimensions of an array due laser structuring depend upon the wavelength, and the spacing between two adjacent elements which is usually close to laser wavelength. Experimentally the average period (d) of the interferometer grating was $1388 \mathrm{~nm}$, which is in agreement with results reported in Chapter 5 . 
The interferometer grating period (d) depends solely on the wavelength of the incident laser, $\lambda$, and the angle between the incident laser and substrate, $\theta$, as in Equation 1-3, which approximately equal to the incident laser wavelength, $\lambda$. If a shorter wavelength laser used in this setup, dots structures of shorter periods or smaller dots are achievable.

On the other hand the (FCW) grating was fabricated by spatial variation of the optical intensity resulting from the interference between the incident laser and the frozen capillary waves which were transiently characteristic to molten silicon and excited by incident laser light scattered parallel to the substrate, initially by the surface roughness and afterwards by the interferometer grating [43]. Experimentally the average dots separation by capillary wave etching was $890 \mathrm{~nm}$. The theoretical period $(\Lambda)$ of $(\mathrm{FCW})$, in a shallow liquid layer can be depicted by Equation 6-1 [75].

$$
\Lambda=\left[\frac{\sigma h}{p}\right]^{1 / 4}\left(2 \pi \tau_{L}\right)^{1 / 2}
$$

Where $\sigma$ is the surface tension coefficient of molten silicon $\left(850 \mathrm{mN} \mathrm{m}^{-1}\right), \rho$ the mass density of liquid layer $\left(2.52 \mathrm{~g} \mathrm{~cm}^{-3}\right), \tau_{L}$ the life time of the molten state (which can be approximated as pulse duration (20 ns), and $h$ is the height of the molten layer (121 nm).

Figure 6-3 $(a, b$ and $c)$ reveals the influence of further increase of dwell time and fluency decrease on dots structures. This leads to as partially as forming dots due to the fluency decrease which is in turn cause decrease the molten layer as in Equation 6-2. The (FCW) period $(\Lambda)$ is influenced by several variables which are very responsive for a tiny change as define by Equation 6-1. It is generally accepted that this type of the dots arises from optical interference effect caused by the interaction between interferometer grating due to interfere of two incident 
beams of fixed angle with a capillary waves which is created at the rough surface during the irradiation and scattered along the surface.

The height of the molten layer was estimated by the Equation 6-2, [76].

$$
h=\frac{\emptyset(1-R)}{\left(C_{v} T_{m}+L_{m}\right)}
$$

Where, $\mathscr{\ell}$, is the laser fluence, $R, C_{v}, T_{m}$, and, $L_{m}$, is given in Table 5-1. The, $h$, is calculated as $121 \mathrm{~nm}$, which is in the same order as the average diameter of the nanodots.

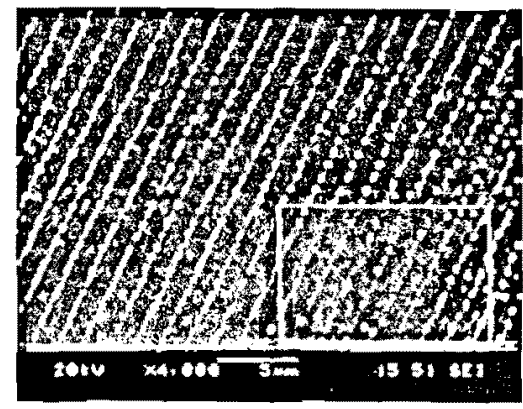

(a)

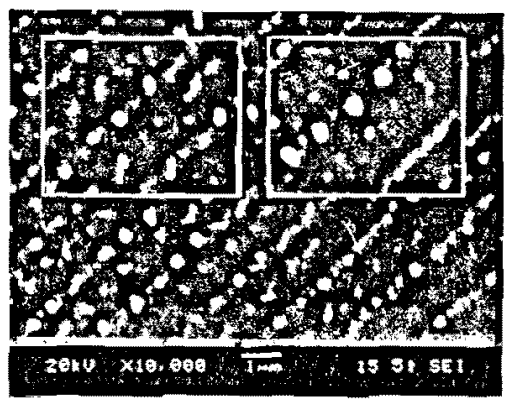

(b)

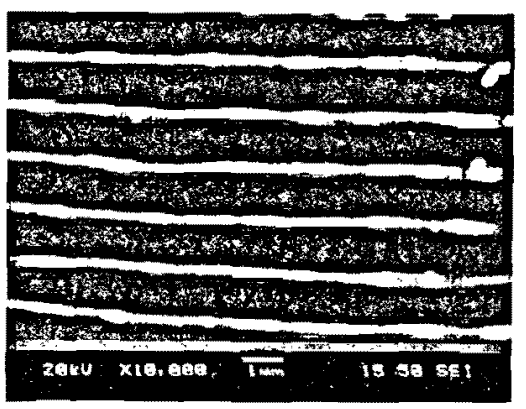

(c)

Figure 6-3: Normal view SEM images of the silicon wafer subjected to Nd: YAG laser pulses at laser fluence of $0.065 \mathrm{~J} \mathrm{~cm}$-2: (a) Shows submicron dots array of silicon surface for partially forming zone, (b) Views of partially structuring of submicron dots at longer dwell time of $150 \mathrm{~ms}, 60 \mathrm{KHz}$, and (c) Lines array in consequence of dots melting at $200 \mathrm{~ms},\left(\lambda=1064 \mathrm{~nm}, \tau_{L}=20 \mathrm{~ns}, 10 \mathrm{KHz}\right)$

Substituting Equation 6-2 in Equation 6-1 and the silicon parameters given in Table 6-1, getting the spatial periodicity $(\Lambda)$ of the grooves that etched by the (FCW) is $897 \mathrm{~nm}$. These results are nearly coincided with the experimental result of $890 \mathrm{~nm}$. The peak to peak splits of interferometer grating (d) is $1390 \mathrm{~nm}$ which was 1.56 time larger than of ( $\mathrm{FCW}$ ) grating spacing $(\Lambda)$ and results in spacious grooves, all grooves are straight, and no vacillation was observed, as shown in Figure 6-2. The submicron etching grooves by (FCW) were registered only at parallel 
beams of fixed angle with a capillary waves which is created at the rough surface during the Irradiation and scattered along the surface.

The height of the molten layer was estimated by the Equation 6-2. [76].

$$
h=\frac{\phi(1-R)}{\left(C_{v} T_{m}+L_{m}\right)}
$$

Where, $\mathscr{O}$, is the laser fluence, $R, C_{r}, T_{m}$, and. $L_{m}$, is given in Table 5-1 The $h$, is calculated as $121 \mathrm{~nm}$, which is in the same order as the average diameter of the nanoduts.

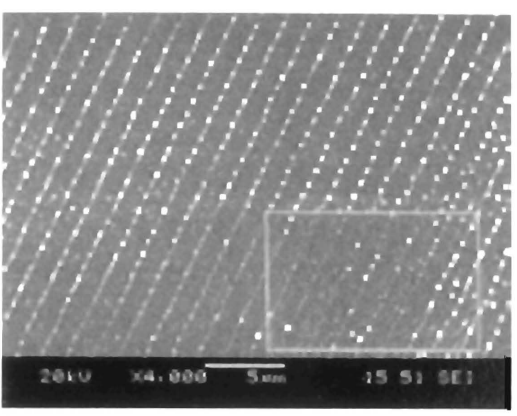

(a)

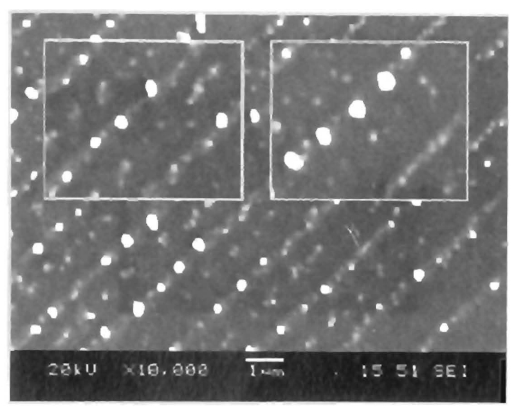

(b)

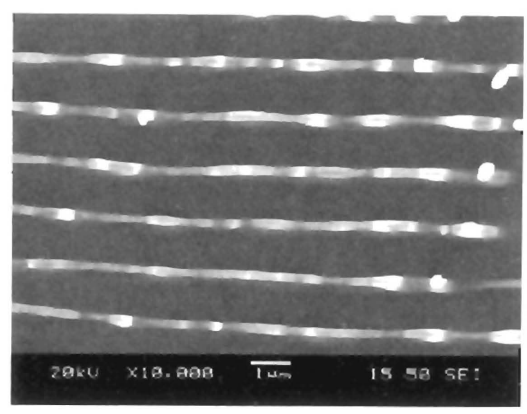

(c)

Figure 6-3: Normal view SEM images of the silicon wafer subjected to Nd: YAG laser pulses at laser fluence of $0.065 \mathrm{~J} \mathrm{cm-2:} \mathrm{(a)} \mathrm{Shows} \mathrm{submicron} \mathrm{dots} \mathrm{array} \mathrm{of} \mathrm{silicon} \mathrm{surface} \mathrm{for} \mathrm{partially} \mathrm{forming} \mathrm{zone,}$ (b) Views of partially structuring of submicron dots at longer dwell time of $150 \mathrm{~ms}, 60 \mathrm{KHz}$, and (c) Lines array in consequence of dots melting at $200 \mathrm{~ms},\left(\lambda=1064 \mathrm{~nm} \cdot \tau_{L}=20 \mathrm{~ns}, 10 \mathrm{KHz}\right)$

Substituting Equation 6-2 in Equation 6-1 and the silicon paramuters given in Table 6-1, getting the spatial periodicity $(\Lambda)$ of the grooves that etched by the (FCW) is $897 \mathrm{~nm}$. These results are nearly coincided with the experimental result of $890 \mathrm{~nm}$. The peak to pcak spiits of Interferometer grating (d) is $1390 \mathrm{~nm}$ which was 1.56 time larger than of (FCW) grating spacing $(\Lambda)$ and rusults in spacinus grooves, all grooves are straight, and no vacillation was observed. as shown in Figuru 6-2. The suhmicron etching grooves by (FCW) were registered only at parallel 
orientation to the irradiated substrate. It may be can explains similar to interference between the laser beams and the scattered beam on the substrate; so surface roughness and in-homogeneity caused surface waves, freezing of capillary waves and generation of a temporary periodic heating pattern during laser irradiation. These waves serve as the starting in-homogeneity of the absorptivity of the surface, as consequence of processes $[43,77,78]$. Take into consideration the fact that $(\mathrm{FCW})$ period $(\Lambda)$ cannot exceed the life time of the liquid layer $\tau_{L}$. The lifetime conditional on the over-heating of the substrate surface above the melting point of a solid and it should be close to laser pulse duration near the melting threshold [75]. Theoretically, nanosecond laser ablation below and close to threshold fluence, the melt has certainly fairly the same duration as the pulse itself, which would be $20 \mathrm{~ns}$ in the available nanosecond laser parameters. Thus, for fluency equal $0.129 \mathrm{~J} \mathrm{~cm}^{-2}$ and $\tau_{\mathrm{L}}$ of $20 \mathrm{~ns}$, the estimated (FCW) Periodicity $(\Lambda)$ which calculated from Equation 6-1 and Equation 6-2 would be equal $897 \mathrm{~nm}$. Expecting due to narrow line-width of interferometer grating, determines the dots diameter decrease in view of a small distribution of sizes. Equation 6-1 and Equation 6-2 predict longer molten state durations can cause melting in the surface substrate with appropriate laser fluence.

\subsubsection{RIPPLES PATTERNS INDUCED BY INTERFERENCE BETWEEN FROZEN CAPILLARY WAVES (FCW) AND LASER BEAM}

Novel method is presented for a single shot laser-patterning of variable features conducting secondary lines on solid surfaces. Employing laser induced grating and secondary patterning; silicon grating with secondary patterning is formed on (C-Si). Direct writing grating used wavelength of $1.064 \mu \mathrm{m}$ and laser beam with p-polarization were performed to characterize the secondary pattern grating as a result of sample optical parameters and energy density (laser 
fluence) by interfering two laser beams. As shown in Figure 6-4, ripples was arose beside and parallel to the interferometer grating periods (d) which formed vertically depends on modified laser polarization by direct writing laser in common optical path configuration. The ripples generated by interference between the incident laser and the frozen capillary waves (FCW). At the same time, ripples were formed in similar manner in vertical direction related to beam polarization of $(\mathrm{P})$ which caused by the interaction by the incident laser with capillary frozen wave. The average ripple spacing $(\Lambda)$ was $270 \mathrm{~nm}$ with orientation parallel to the interferometer grating patterns. Ripple formation happened by same phenomenon which expressed by Equation 6-1 and Equation 6-2. No dots arose in this case, no junction between two different patterns which formed by same orientation vertically to $\mathrm{X}$-axis of silicon wafer.

The forming of a ripple structure on $\mathrm{Si}$ was such as regular pattern with spacing $(\Lambda)$ of $270 \mathrm{~nm}$ in the average. The ripples structure was shaped at the valley spot between two lines of interferometer grating at one grating period. The dimensions of ripples spacing $(\Lambda)$ due laser structuring depend upon the laser fluence intensity of $0.015 \mathrm{~J} / \mathrm{cm}^{2}$ and pulse duration of $20 \mathrm{~ns}$ at repetition rate of $30 \mathrm{KHz}$ with machining spot size of $40 \mu \mathrm{m}$. Theoretically, the ripples period $(\Lambda)$ of secondary pattern is given by the Equation 6-1 and Equation 6-2 [75, 76]. 

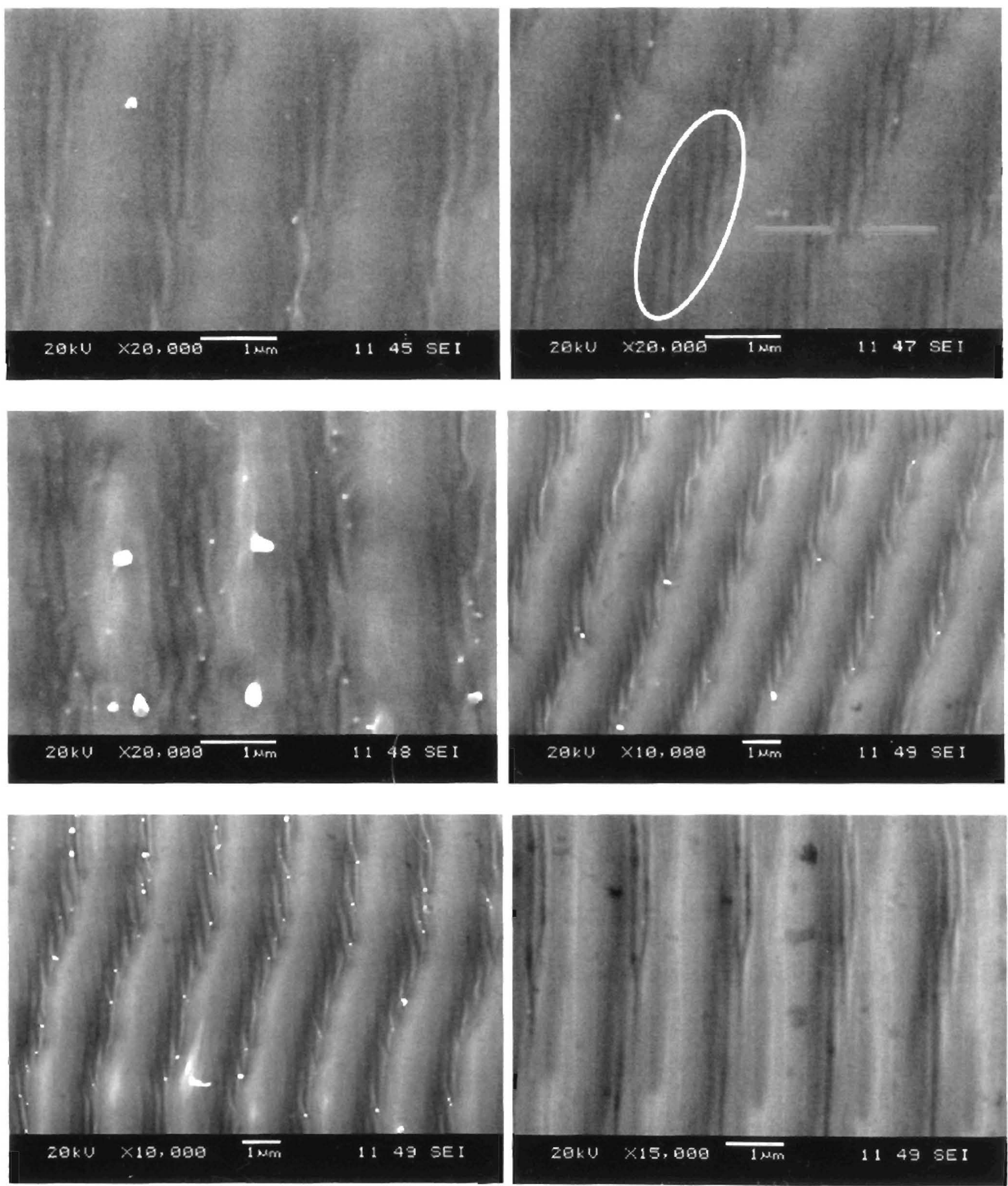

Figure 6-4: SEM views of ripples at repetition rate of $30 \mathrm{KHz}$ with pulse energy of $0.199 \mu \mathrm{J}$. 


\subsection{PRACTICAL CONSIDERATION}

\subsubsection{PROSSESSING PARAMETERS}

\subsubsection{Effect of Pulse Repetition Rate}

As seen in Figure 6-5 ( $a$ and $b$ ) the effect of pulse repetition rate on ablation threshold. Graphs demonstrating the threshold energy fluence and threshold average beam power for different laser repetition rates. Figure 6-5 (a) shows the threshold fluence decreases rapidly with increase in the laser repetition rate. This result agrees well with those obtained by other authors [79]. However, the higher the laser repetition rate is, the higher the threshold average beam power becomes as shown in Figure 6-5 (b).

It is important to note that Figure 6-5 (a) flats out in the range of repetition rate between $10-30 \mathrm{KHz}$ and remains at a constant threshold fluence. This is due to the fact that minimum threshold fluence is required to start the ablation process. If the laser fluence goes below this minimum threshold fluence of $10 \mathrm{KHz}$, no ablation occurs no matter how much machining time is fed to the target surface. Therefore, as Figure 6-5 (b) demonstrates, while the threshold fluence remains almost constant, increase in the pulse repetition rate leads to the increase in threshold laser power. 


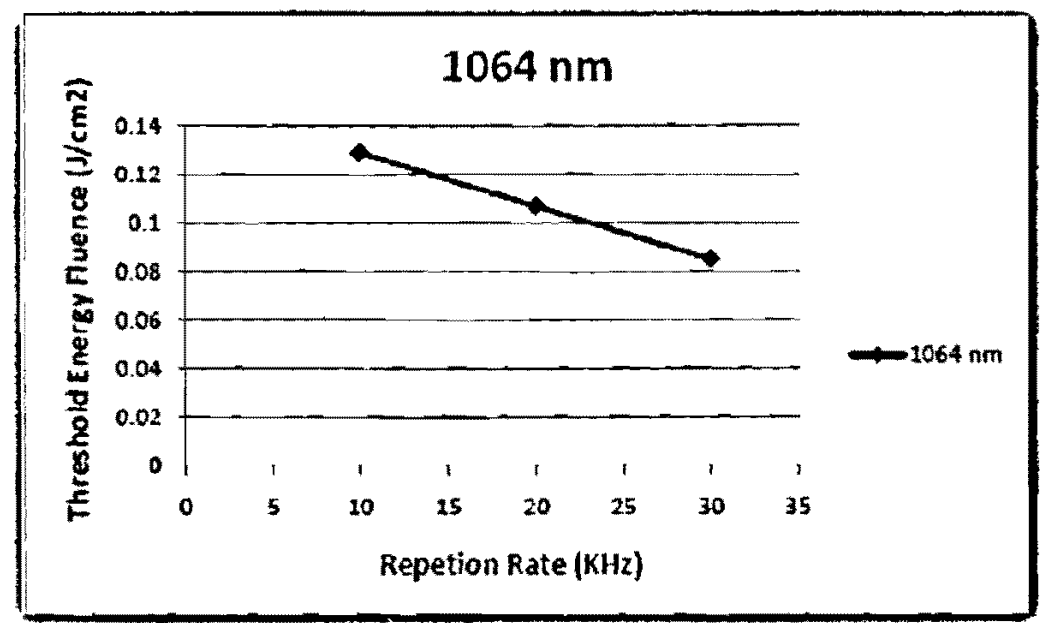

Figure 6-5 (a): Threshold Energy Fluence vs. Repetition Rate from $10 \mathrm{KHz}$ to $30 \mathrm{KHz}$

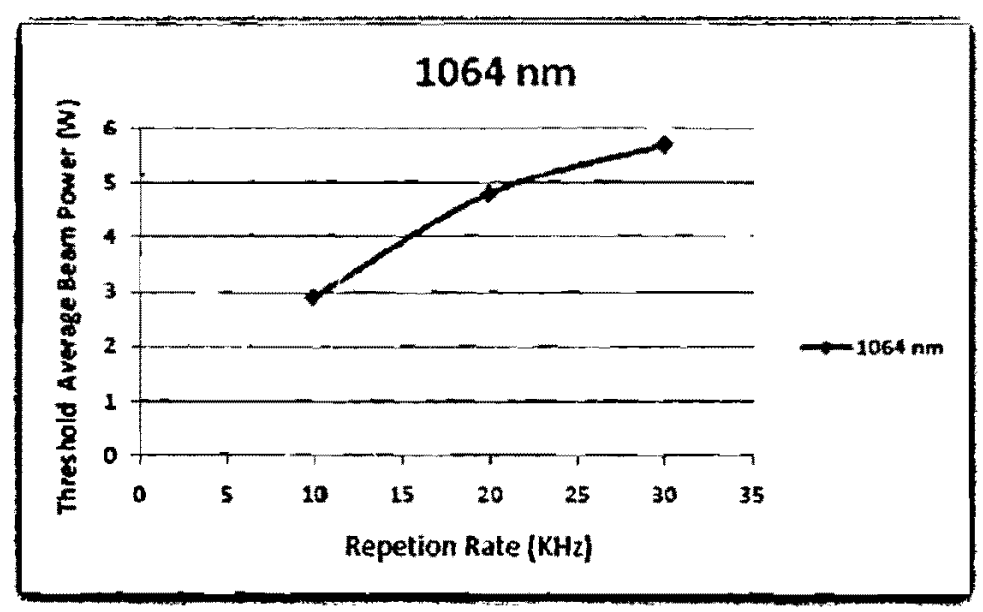

Figure 6-5 (b): Threshold Average Beam Power vs. Repetition Rate from $10 \mathrm{KHz}$ to $30 \mathrm{KHz}$

If these estimates are confirm the indication that the sub-micrometer structures obtained was practically frozen capillary waves (FCW), characteristic to molten silicon and suggested that surface waves created by the laser pulses can be responsible for the phenomenon. 


\begin{tabular}{|l|c|c|}
\hline \multicolumn{1}{|c|}{ Silicon Parameter } & Valua & Reference \\
\hline Reflectivity $\mathrm{R}$ & 0.3 & {$[80]$} \\
\hline Specificheat capacity $\mathrm{C}, \mathrm{J} \mathrm{\textrm {cm } ^ { - 3 } \mathrm { K } ^ { - 1 }}$ & 2 & {$[70]$} \\
\hline Metting temperature $\mathrm{Tm}, \mathrm{K}$ & 1685 & {$[76]$} \\
\hline Specificheat of malting $\mathrm{Lm}, \mathrm{cm}^{-3}$ & 4130 & {$[80]$} \\
\hline Surface tension coefficient $0, \mathrm{mN} \mathrm{m}^{-1}$ & 850 & {$[75]$} \\
\hline Liquid mass density $\mathrm{g} \mathrm{g} \mathrm{cm}^{-3}$ & 2.52 & {$[81]$} \\
\hline
\end{tabular}

Table 6-1: Silicon parameter used to solve the Equation 6-1 and Equation 6-2

Thus, this method of using laser induced (FCW) etching will be feasible for fabrication of submicron dots structures with unique physical properties such as quantum limitation to reduce the structure dimensions. The bright color of the dots refers to silicon dioxide which was prior to irradiation and consequence of silicon exposure to air [82].

\subsubsection{Secondary Ripples Formation Mechanisms}

As mentioned by $[10,74]$ after irradiation of silicon surface with linearly polarized the ripples will form at stages of amorphization and annealing before arrive at threshold ablation stage. Therefore in the next sections, several mechanisms for formation of ripples have been proposed. Surface tension waves, surface tension gradient, Acoustic waves, interference between the incident laser beam with surface electromagnetic waves (SEW), interference between the incident laser and the frozen capillary waves (FCW) mechanisms, have been described in detail. 


\subsection{Surface Tension Waves}

As mentioned by [83], related to an ideal irrotational and incompressible fluid, the dispersion relation for surface tension waves is given by Equation 6-3. This Equation can be used to estimate ripple periodicity,

$$
\omega=\left[\left(k^{3} / \rho\right) \tanh (k h)\right]^{1 / 2}
$$

Where, $\gamma$, is the surface tension, $k$, is the wave number. The angular frequency is assumed to be $2 \pi$ times the inverse laser pulse width. Equation $6-3$ is quite insensitive to the height of molten silicon. For [(hk) $>>1$ ], Equation $6-3$ is independent of $(h)$ and for $(h k<<1)$, capillary wavelength $\lambda$ varies as $h^{1 / 4}$.

\subsection{Surface Tension Gradients}

Throughout laser surface melting, temperature gradients on the melt surface between the laser beam impact point and the intersection line of the solid liquid interface with the surface lead to surface tension gradients that sweep liquid away from the beam impact point. The temperature gradient extends radially away from the center of the laser beam. Under the laser beam, the temperature of the liquid is at the highest and surface tension of the liquid is at the lowest. As the liquid temperature decreases away from the center of the beam, the surface tension of the liquid increases. This phenomenon causes the liquid to flow away from the center of the beam and therefore depress the liquid surface underneath the laser beam and ridge the liquid surface elsewhere. As the beam passes to other areas of the surface, the height difference between the liquid under the beam and the liquid away from the beam increases. A pressure head 
induces a counter flow of liquid back to the region underneath the beam. At steady state, the liquid flow away from the beam caused by the surface tension gradient and the gravity counter flow of the liquid towards the beam balance each other out. Due to the large temperature gradients and rapid solidification rates associated with laser surface melting, this distortion of the liquid surface becomes frozen into the surface and ripples appear. The only manner in which these ripples from surface tension gradients can be avoided is if the laser beam sweep velocity exceeds a certain critical velocity. The various stages of the laser beam sweep and the creation of ripples due to surface tension gradients obey the Equations which states the critical velocity of the laser beam must exceed, as the following [84],

$$
V_{C}=\frac{1}{2} w / \tau
$$

Where, $w$, is the ripple base width, $\tau$, is the time required to form ripples.

It should be noted that, $\tau$, can obtain by the Equation 6-5,

$$
\tau=\left[\frac{3 w}{g}\right]^{1 / 2}
$$

Where, $g$, is the acceleration field. Using Equations 6-4 and 6-5, $V_{c}$, was expressed as,

$$
V_{C}=\left[\frac{w g}{12}\right]^{1 / 2}
$$

The surface tension, $\sigma$, of a liquid may be written as $\left(\sigma=\sigma_{\mathrm{o}}-s T\right)$. Here, $\sigma_{\mathrm{o}}$ is the surface enthalpy, $s$, is the surface entropy and, $T$, is the temperature. Since the surface entropy $(s)$ is positive, the temperature coefficient $\mathrm{d} \sigma / \mathrm{dT}$ of the surface tension of most liquids is negative i.e. ( $\sigma / \mathrm{dT}<0$ ). However, a higher beam velocity requires a higher power laser in order to achieve 
the same penetration depth. The high power laser leads to additional cost and also surface rippling through other mechanisms such as vapour pressure depression of the melt surface.

\subsection{Acoustic Waves}

According to $[83,85]$, silicon goes through volume contraction and generates an acoustic wave upon melting. The thermo elasticity of silicon creates the acoustic wave even before melting with a power spectrum that peaks around inverse of the pulse duration. Higher above the threshold the generated sound wave lasts over several periods and consequently washes out. The acoustic wave propagates on the surface in an anisotropic manner. In [85], the ripples appeared parallel to one of the (110) Si crystal axes. However, in [83], the ripples are seen to be perpendicular to the laser polarization. The ripples are in fact a frozen out surface acoustic wave with a frequency of inverse the pulse width of the illuminating laser.

\subsection{Interference the Incident Laser Beam With Surface Electromagnetic Wave} (SEW)

According to [86] observes laser induced periodic surface structures (LIPSS) in term of ripples. Author stated that after exposure to linearly polarized radiation at normal incidence, the lateral period of the observed surface structures is very close to the wavelength of the incident radiation. It is generally accepted that this type of wavelength ripple arises from optical interference effects due to the superposition of the incident radiation with a surface electromagnetic wave (SEW) which is created at the rough surface during the irradiation and which is scattered along the surface. Like this theory takes into consideration the interaction of an electromagnetic wave with a microscopically rough surface, which includes also the possible 
excitation of surface polaritons. This theory predicts possible (LIPSS) wave vectors $k$ of the surface [with $|\mathrm{k}|=(2 \pi) / こ$ ] as a function of the laser parameters (angle of incidence $\theta$, polarization direction, and wave vector of the incident radiation $\mathrm{k}_{\mathrm{L}}\left[\right.$ with $\left.\left|\mathrm{k}_{\mathrm{L}}\right|=(2 \pi) / \lambda\right]$ which has a component $\mathrm{k}_{\mathrm{i}}$ in the surface plane and surface parameters as dielectric constant and surface roughness. It leads to an expression for the inhomogeneous energy deposition into the irradiated material which is proportional to $n\left(k, k_{i}\right) \times|b(k)|$, where $n$ is a response function describing the efficacy with which the surface roughness at $\mathrm{k}$ leads to inhomogeneous absorption of radiation. The second factor $b$ represents a measure of amplitude of the surface roughness at $k$ which is a slowly varying function for a surface with a homogeneously distributed roughness.

As [87] illustrates, the periodic ripple structures are formed by the spatial modulation of optical intensity resulting from the interference between the incident laser light and (SEW).

A variety of different (SEW) surface modification have ripple structures can be achieved by this fabrication method based on polarization dependence. The (SEW) structures with a high spatial frequency are attractive, but unsuitable for application to microelectronic devices. The orientation and spatial period of the (SEW) structure are determined by the wavelength, the polarization, and incident angle of the beam. The orientation of the (SEW) grating structure is determined exclusively by the polarization of light. In particular, when using s-polarized light the grooves of the (SEW) grating run perpendicular to the vector of the incident electric field. In the case of using p-polarized light, the grooves of the SEW grating run parallel to the vector of the incident electric field.

The theoretical period of SEW ripple obtained by using P-polarized laser is described as [87], 


$$
\square_{s}=\frac{2 \pi}{k_{s}+k_{0} \sin \theta}
$$

Where, $k_{o}$, is the wave number of incident laser light and, $k_{s}$, the real part of the propagation (SEW) wave vector. If, $\square_{h}$, is an integer multiple of, $\square_{s}$,

$$
\Sigma_{\mathrm{h}}=\mathrm{m} \cdot \Xi_{\mathrm{s}}
$$

Where, $m$, is an integer

The spatial periods of (SEW) ripples are determined by the incident angle and wave number of incident light. Thus, the spatial period $(\Lambda)$ of these ripples is known to be closely related to the wavelength number and angle of incidence.

\subsection{Interference between the Incident Laser and the Frozen Capillary Waves (FCW)}

A frozen capillary wave was as interest mechanism for many groups in laser induced ripple structures. According to [75] illustrates, the large scale periodic structures on a single crystal Si surface. It is suggested that the spatial period of the structures is determined by the capillary wave period on the molten solid surface. These waves serve as the starting inhomogeneity of the absorptivity of the surface. The capillary waves can be responsible for the formation of the initial periodic structure on the surface of molten $(\mathrm{C}-\mathrm{Si})$. The dispersion relation

- for shallow capillary waves on a liquid surface for duration of laser pulse near threshold melting $\left(\tau_{\mathrm{p}}=20 \mathrm{~ns}\right)$ are given by the following expression,

$$
\omega^{2}=\left(\frac{\sigma}{\rho}\right) h k^{4}
$$


The period of the wave as the time of existence of the melt, $t$, is expressed by,

$$
t=2 \pi / \omega
$$

This time depends on the overheating of the surface above the melting point of a solid and should be close to the duration of the laser pulse near the threshold of melting, therefore $t \approx$ $\tau_{\mathrm{p}}=20 \mathrm{~ns}$.

$$
\begin{aligned}
& \text { Also for, } k \text {, it's given by, } \\
& \mathrm{k}=2 \pi / \lambda
\end{aligned}
$$

Substituting Equation 6-10 and Equation 6-11 in Equation 6-9 will get the relation by expression of wavelength, $\lambda$, as follow,

$$
\lambda=\left[\frac{\sigma h}{\rho}\right]^{1 / 4}\left(2 \pi \tau_{L}\right)^{1 / 2}
$$

Where, $h$, is the depth of the molten liquid, $\omega$, is the frequency of the wave, $\sigma$, is the coefficient of surface tension of the melt, $\rho$, mass density of the liquid layer, $k$, stands for the wave vector of the capillary wave, and, $\lambda$, is the wavelength of the capillary wave.

Group [88] demonstrated that the periodic ripple structures on silicon surface exposed in air to $1064 \mathrm{~nm}, \mathrm{Nd}$ : YAG pulsed laser irradiation. They are reached to same as results as of [75]. The group put to considering the fact that the capillary wave period cannot exceed the life time of the molten state $\tau_{\mathrm{L}}$, and therefore the average microstructures ripples spacing $(\Lambda)$, can be estimated instead by the expression as in Equation 6-1. Finally, the estimation of the height of 
the molten layer (h) in Equation 6-1, can get it by the Equation 6-2, according to [89] which have suggested that $(\mathrm{FCW})$ is responsible for ripples forming.

In the experiment all the indicators for "dots formation" attributed to the theory of interference between the incident light and the capillary surface wave which effect on the molten silicon layer height and dots size. As $[75,88,89]$ demonstrated that the formation of ripple using pulsed laser below threshold energy fluence was related to the theory of "interference between the incident laser and the frozen capillary surface wave". However, our experimental results show for higher applied laser fluence, the high pulse energy at $10 \mathrm{KHz}$ induced ripples separations. For instance, the experimentally result of frozen capillary wave separation equal 890 $\mathrm{nm}$, this values so far from the applied laser wavelength of $1064 \mathrm{~nm}$. So, theoretically it can only apply the Equation 6-1 and Equation 6-2 in order to expedience both theoretically $897 \mathrm{~nm}$ and experimentally $890 \mathrm{~nm}(\mathrm{FCW})$ reading. Therefore for nanosecond laser pulse duration that was used, the above "SEW and acoustic wave" mentioned mechanisms are not responsible for formed ripples on silicon surface.

\subsection{SUMMARY}

The study in this Chapter has investigated the capability of using a low power nanosecond laser for silicon sample. Nanosecond laser of $1064 \mathrm{~nm}$ wavelength with high energy density combined with the low repetition rate in the range of $10-70 \mathrm{KHz}$ was presented for $(\mathrm{C}-\mathrm{Si})$ patterning. This method attains new concept to fabricate periodic submicron dot structures of 240 $\mathrm{nm}$ diameter (dd) in average fabricated directly by a single step maskless process. The period (d) of the etched groove by the interferometer grating was $1390 \mathrm{~nm}$, while the spatial periodicity $(\Lambda)$ of the groove by the frozen capillary wave (FCW) etching was $897 \mathrm{~nm}$. The influence of the 
machining time, pulse energy and laser parameters on the machining quality and the feature size were studied successfully. The etched depth of interferometer grating increased linearly in time, while the depth of the (FCW) grating was increasing exponentially with increasing laser irradiation time. Based on our knowledge, it was first time that the low-repetition-rate nanosecond laser has been demonstrated industrial applications for submicron dot structures. Colliding two beams and generating interference fringes to fabricate submicron dots arrays by nanosecond laser the concept is did not demonstrate before. Such as this technique the submicron dots formation, patterning and orientation obey the grating which generated by self-aligned common path optical configuration interference and the surface roughness capillary wave (FCW) growth Equations. The laser induced by (FCW) etching of direct ablation technique, which offers substantial advantages over conventional lithographic techniques in procedure flexibility, speeds, and simplicity. This technique for dots array structures fabrication can be realized for commercially usage if the beam quality of shorter wavelength lasers is further improved. Future work would comprise increase in the dots density per area, reduction of the dots size and more control for the orientations.

The formation of the submicron dots and the dynamics of the interaction between the nanosecond laser and Silicon have been discussed. An in-depth study was carried out in which of the laser parameters influence on the profile and quality of fabricating sub-micron dots patterns. Several mechanisms for formation of ripples have been proposed. The preferred mechanism which responsible for ripple structure in nanosecond regime was interference between the incident laser and the (FCW) theory, have been described in detail. 


\section{CHAPTER 7}

\section{LASER PATTERNING USING SECOND HARMONIC GENERATION WAVELENGTH (SHG)}

\subsection{INTRODUCTION}

In this Chapter, a novel method is developed for a single shot laser patterning of variable nano features on a thin gold film grating of $1000 \AA$ thickness coated on quartz substrate. Direct writing grating in common optical path setup that uses second harmonic wavelength were performed for grating fabrication. The grating characterize was a result of sample optical parameters and laser power density of interfering two laser beams in common optical path configuration.

Driven by the potential applications in optical communications and displays, a considerable research effort has been ongoing in fabricating sub wavelength structures with periods smaller than $200 \mathrm{~nm}$. Sub $200 \mathrm{~nm}$ gratings have the potential of replacing the conventional optical multi layer elements [96], such as anti-reflection surface, polarization splitter, and resonant filter, with improved quality. Usually, these gratings should be written either in transparent materials or on optical thin films. The standard fabrication process involves interferometric lithography; evaporation and dry etch [97]. The end product will then be used as a master to perform the standard replications of sub wavelength structures.

There are numerous different approaches to generate sub wavelength gratings, including (UV) lithography [98], e-beam lithography [99] and nano-imprint lithography [100]. Nanosecond 
and femtosecond lasers have become promising tools for inscribe gratings; primarily due to their unique properties of photon-matter interaction [20] which makes material removal can be precisely controlled in sub-wavelength scale [101]. Several attempts of using direct writing of laser interference for surface relief grating writing have been made $[48,55,102]$.

\subsection{EXPERIMENTAL RESULTS}

\subsubsection{GRATINGS INDUCED BY LONG PULSES NANOSECOND LASER OF $532 \mathrm{NM}$ USING COMMON OPTICAL PATH INTERFERENCE}

Interferometric grating patterning is based on the formation of stable and uniform interference fringes. This task demands light sources of high temporal and spatial coherence. Typical Nd: YAG lasers, produce high energy density in the UV region. Fabrication setup was used pulse duration of $20 \mathrm{~ns}$ of second harmonic generation wavelength of $532 \mathrm{~nm}$ that generates low laser energy of $0.152 \mu \mathrm{J}$. Laser patterning of grating of $260 \mathrm{~nm}$ period were conducted on silicon wafer. The morphology of the samples was characterized and studied with the help of a (SEM).

Laser parameter was used is P-polarization of $50 \mathrm{KHz}$ repetition rate nanosecond laser. The effect of using high repetition rate of $50 \mathrm{KHz}$ shows that grating edges and line width is more uniform. The feature size of grating period is $260 \mathrm{~nm}$ is less than half wavelength of 532 $\mathrm{nm}$ that used. This result can consider as a great achievement when nanosecond laser is used; in spite of HAZ impress and some of separated debris is explicit. Figure 7-1 displays grating

structure of period $260 \mathrm{~nm}$, arose through interfere two laser beams in common optical path configuration. 

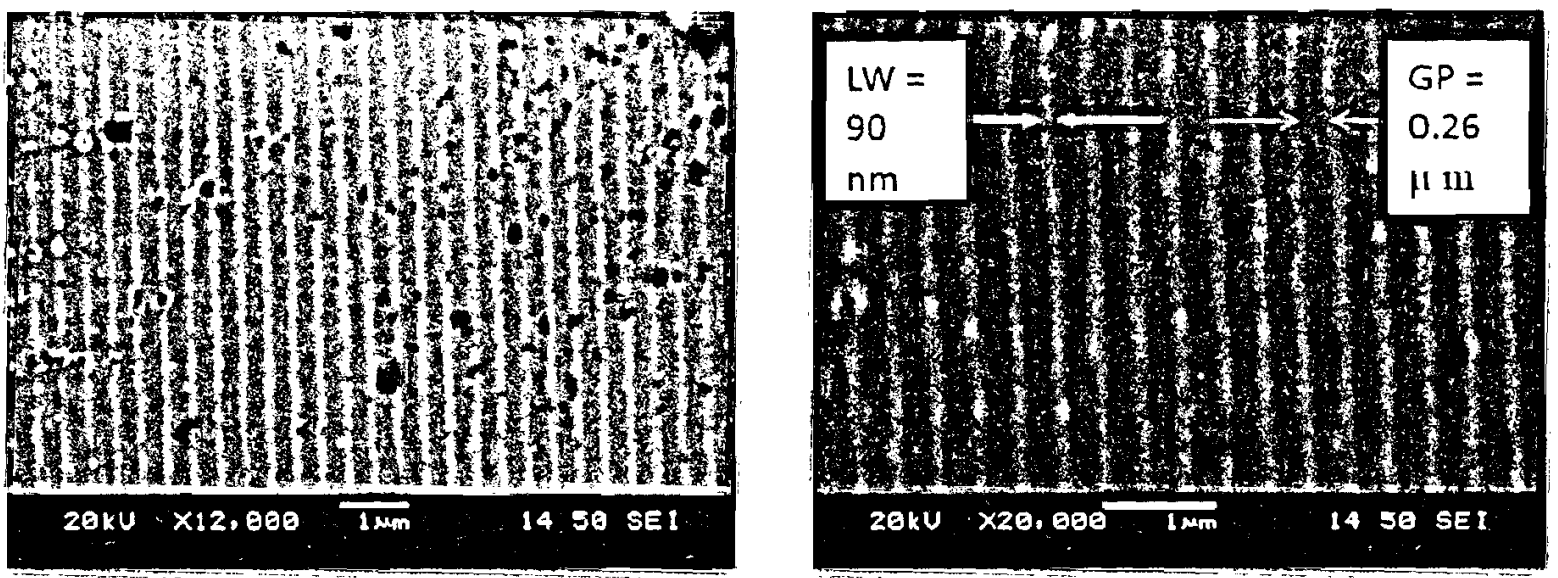

Figure 7-1: shows SEM image of the grating fabricated using p-polarization of second harmonic generation of $532 \mathrm{~nm}$ of wavelength at repetition rate of $50 \mathrm{KHz}$, pulse duration of $20 \mathrm{~ns}$, and (LW) of $90 \mathrm{~nm}$ and $(G P)$ of $260 \mathrm{~nm}$

\subsubsection{GRATINGS PATTERN INDUCED BY ULTRASHORT FEMTOSECOND PULSES LASER OF $400 \mathrm{NM}$}

In this experimental study, a novel direct patterning technique for gratings fabrication on thin gold film surface was presented. The pattern arose by interfere two ultrashort laser beams in a common optical path configuration. The grating fabrication by femtosecond laser was employed the same setup and concept for common optical path which used in Chapter 5. The most challenge for using short pulses femtosecond laser in term of laser interference is time delay or the difference between two interfering beams (two interfering pulses) which should not exceed $45 \mu \mathrm{m}$ in our parameters. Otherwise the setup will not meet the criteria for suitable fringes pattern generation. Since light speed is $2.8 \times 10^{9} \mathrm{~m} / \mathrm{s}$. If light speed will multiply by femtosecond pulse duration of $150 \mathrm{fs}$, this will give certain small value of $45 \mu \mathrm{m}$. While for long pulses nanosecond laser the problem disregards and paths difference will be much bigger of 56 m. Hence two pulses time delay in femtosecond laser system is very limited and should not be 

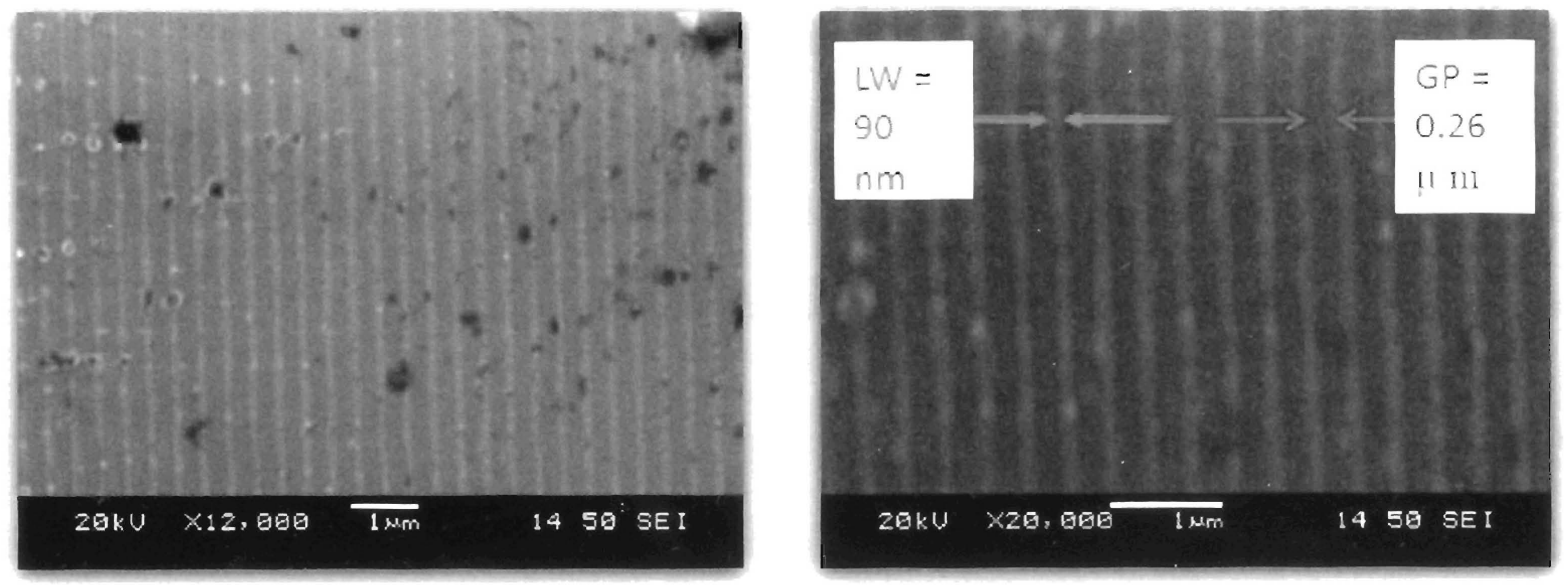

Figure 7-1: shows SEM image of the grating fabricated using p-polarization of second harmonic generation of $532 \mathrm{~nm}$ of wavelength at repetition rate of $50 \mathrm{KHz}$, pulse duration of $20 \mathrm{~ns}$, and (LW) $G_{j}^{5}$ $90 \mathrm{~nm}$ and $(G P)$ of $260 \mathrm{~nm}$

\subsection{GRATINGS PATTERN INDUCED BY ULTRASHORT FEMTOSECOND PULSES I. ASER OF 400 NM}

In this experimental study, a novel direct patterning wechnique for gratings fubrication on thin gold film surface was presented Thu pattern arosc by inierfere two ultrashori laser buams in a common optical path configuration. The grating fabrication by femiosecond laser was smployed the same setup and concept for common optical path which used in Chapter 5. The most challenge fur using short pulses femtosecond laser in term fl laser interference is timu delay or the difference between two interfering beams (two interfering pulses) which should not exceed $45 \mu \mathrm{m}$ in our parameters. Otherwise the sutup will not meet the criteria for suitable fringes pattern generation. Since light speed is $2.8 \times 10^{9} \mathrm{~m}$ 's. If light speed will multiply by femtosecond pulse duration of $150 \mathrm{fs}$, this will give certain small value of $45 \mu \mathrm{m}$. While for long pulses nanosecond laser the problem disregards and paths difference will be 1. unch bigger of 56 m. Hence two pulses timu delay in femtosecond laser system is very limited and should not be 
greater than $45 \mu \mathrm{m}$. The fabrication technique is simplifies the optical setup, immune to extraneous, good edge acuity and inherent vibration. The morphology of the samples surfaces was characterized with the help of atomic force microscope (AFM).

As in Figure 7-2, the experimental result that induced by femtosecond laser system which delivered pulses at a central wavelength of $800 \mathrm{~nm}$ and pulse duration of $150 \mathrm{fs}$ at repetition rate of $1 \mathrm{kHz}$. The emitting laser beam was linearly polarized and had a diameter of $5 \mathrm{~mm}$. A second harmonic generator was used to double the laser frequency to $400 \mathrm{~nm}$.

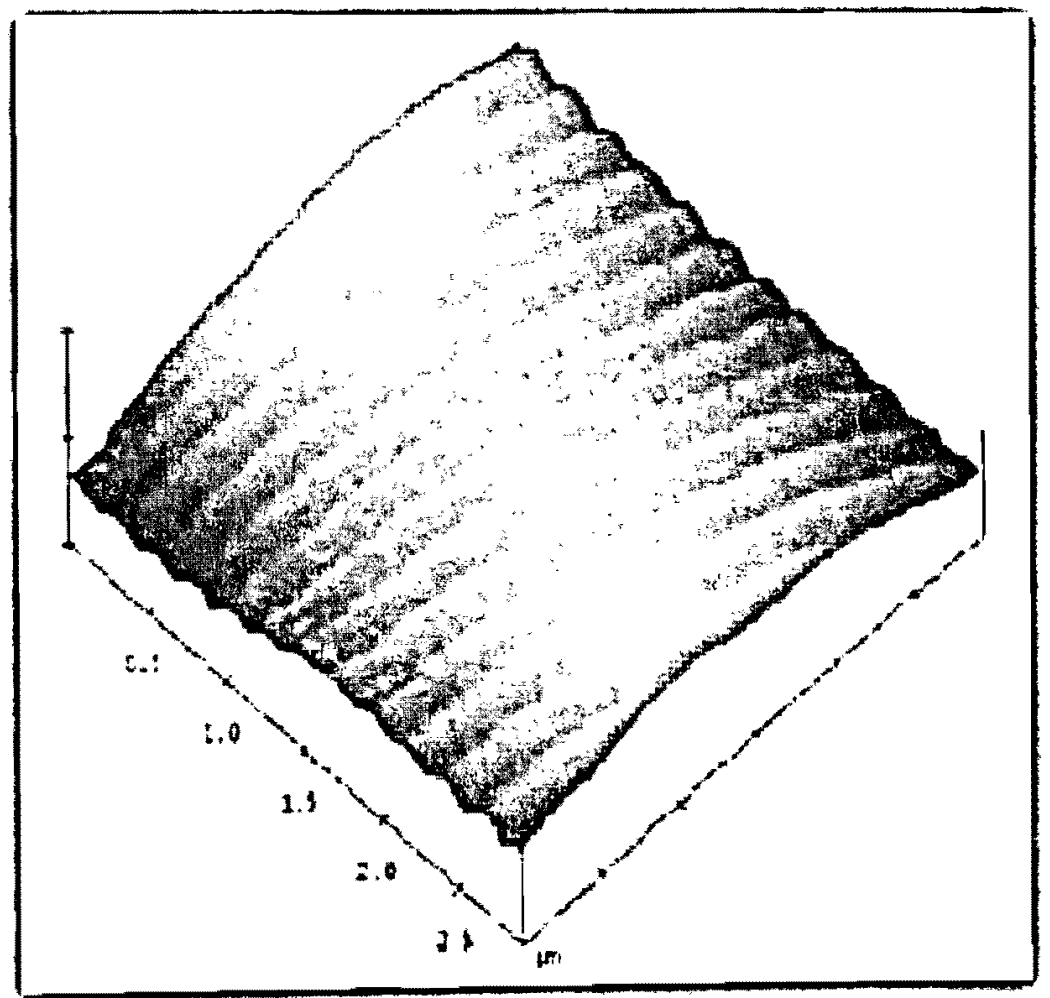

Figure 7-2: shows AFM image of the grating formed at polarization parallel to the propagation plan employed second harmonic generation wavelength of short pulse femtosecond laser of $400 \mathrm{~nm}$ and short pulse duration of $150 \mathrm{fs}$

Figure 7-2 presents an (AFM) image of a grating formed with common optical path setup. The intensity of the pulses was controlled far below the ablation threshold; otherwise the 
greater than $45 \mu \mathrm{m}$. The fabrication technique is simplifies the optical setup. immune to extraneous, good edge acuity and inherent vibration. The morphology of the samples surfaces was characterized with the help of atomic force microscope (AFM).

As in Figure 7-2, the experimental result that induced by femtosecond laser system which deliverid pulses at a central wavelength of $800 \mathrm{~nm}$ and pulse duration of $150 \mathrm{fs}$ at repetition rate of $1 \mathrm{kHz}$. The emitting laser beam was linearly polarized and had a diameter of $5 \mathrm{~mm}$. A second harmonic generator was used to double the laser frequency to $400 \mathrm{~nm}$.

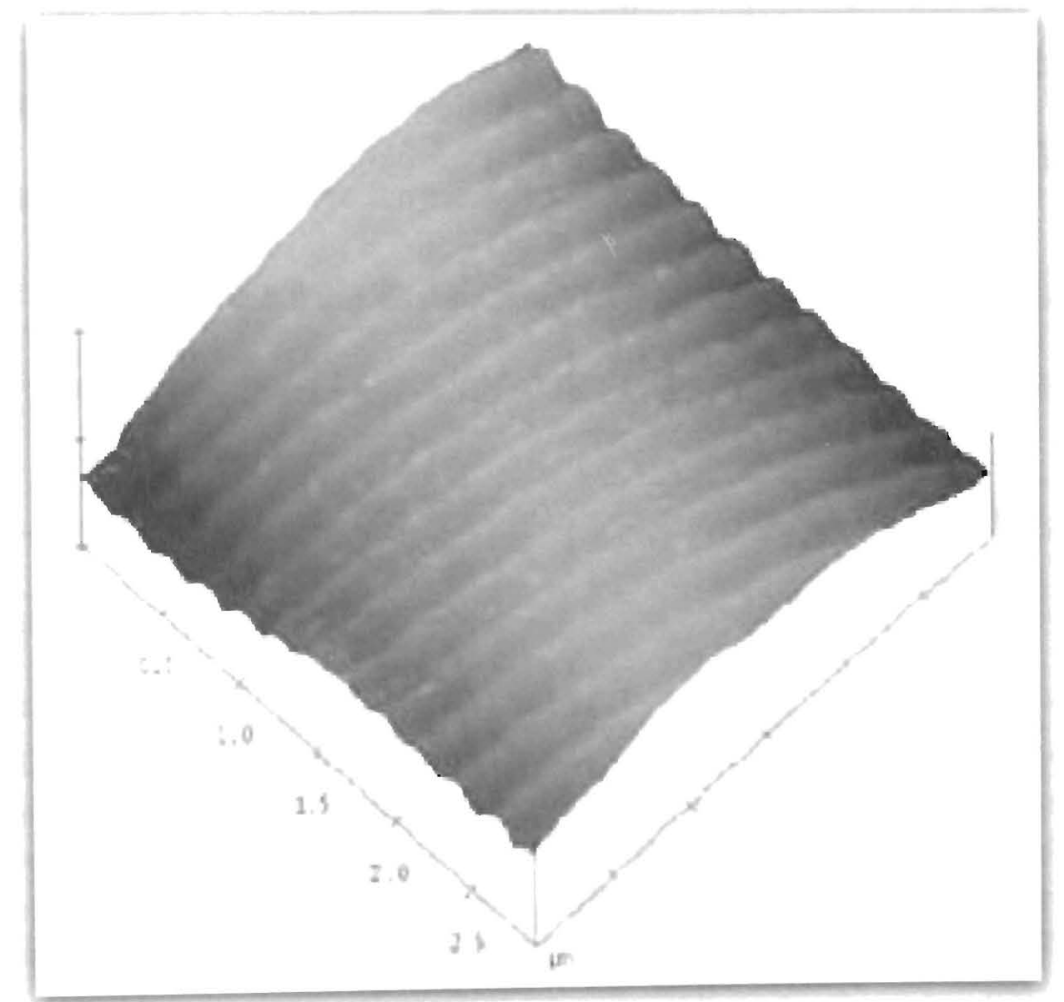

Figure 7-2: shows .1FM image of the grating formed at polarization parallel to the propagation plan employed second harmonic generation wavelength of short pulse femtosecond laser of $400 \mathrm{~nm}$ and short pulse duration of $150 \mathrm{fs}$

Figure 7-2 presents an (AFM) image of a grating formed with cummon upticai path setup. The intensity of the pulses was controlled far bolow the ablation threshold; otherwise the 
high thermal transports during the laser interaction will wash out the finely structured grating. The fringe orientation is parallel to beams overlapping direction (the propagation plane) which fulfilled by controlling the polarization of the incident beam and rotates to the direction parallel to the propagation plane. The profile of the grating was measured by (AFM) and is given in Figure $7-3$. The depth of the grating is about $40 \mathrm{~nm}$. The period (d) of the fabricated grating was measured as $220 \mathrm{~nm}$, which is nearly agrees with half wavelength of the incident laser beam. Leaping to shorter wavelength than $400 \mathrm{~nm}$ at $3^{\text {rd }}$ harmonic wavelength, the feature size become smaller, and so for every H.G., the feature size will goes to half the wavelength.

\subsubsection{PROCESSING PARAMETERS}

\subsubsection{Effect of Wavelength}

To induce femtosecond laser interference, pulses must temporally and spatially overlap. The optical path difference $(\Delta d)$ for two pulses expressed by Equation 7-1[103],

$\Delta \mathrm{d}<c \tau$ Eq 7-1

Where, $c$, is the light speed and, $\tau$, is the pulse duration. For example to interfere $150 \mathrm{fs}$ laser pulses, the optical path difference between two interfering beams should not be greater than $45 \mu \mathrm{m}$. This number can be very critical value to superpose two beams at same focal plane and raise fringes pattern. 


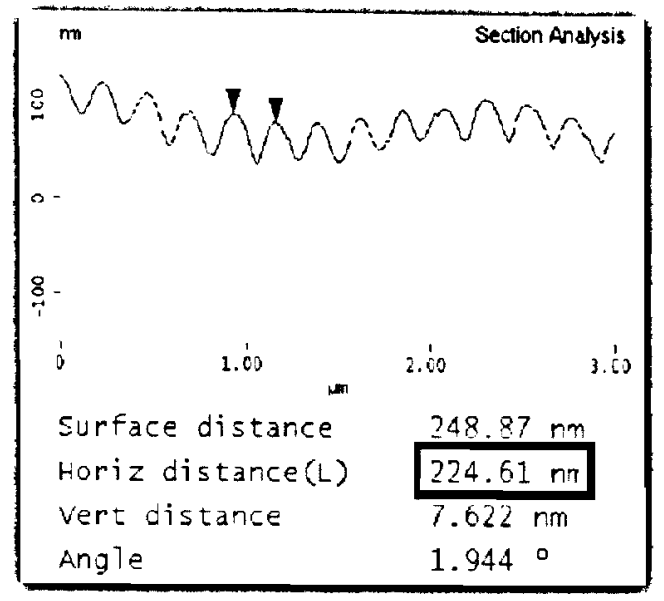

Figure 7-3: The grating period is measured as about $220 \mathrm{~nm}$ that induced by femtosecond laser of 400 ns wavelength

The number of fringes produced by the interference of two beams can be controlled by varying laser wavelength to smaller harmonic generation or frequency. This method offers a femtosecond laser technique for gratings fabrication by direct writing in a common optical path configuration without difficulty in beams interfering. Their offers precisely control the optical path difference.

\subsection{SUMMARY}

In this Chapter grating fabrication by laser interference in common optical path configuration has been proposed. The two beams interference for a surface grating by using second harmonic generation for both nanosecond and femtosecond laser have been demonstrated. Initially nanosecond laser has pulse duration of $20 \mathrm{~ns}$ and low laser energy of $0.152 \mu \mathrm{J}$ was employed. Laser patterning of grating of $260 \mathrm{~nm}$ period were conducted on silicon wafer by second harmonic generation of $532 \mathrm{~nm}$ wavelength. Laser was polarized to Ppolarization of $50 \mathrm{KHz}$ repetition rate. The morphology of the samples was studied by (SEM). 
Secondly, direct writing of half wavelength gratings with simple optics by interference femtosecond pulses on transparent substrate coated with thin gold film. Equation 1-3 was employed to calculate grating period (d). Surface grating on gold film was conducted in atmosphere of period (d) $220 \mathrm{~nm}$. Femtosecond laser has short pulse duration of 150 fs for second harmonic generation wavelength of $400 \mathrm{~nm}$. The result was examined by (AFM).

This technology used common optical path configuration was applied for femtosecond laser fabrication. The beams automatically interfered and it does not require complicated optical setup. The optical setup is immune to extraneous and inherent vibration; it is fast and consists of little optics. This fabrication technique can be realized commercially. Future work would involve reduction of the feature size and increase in the frequency of the grating. 


\section{CHAPTER 8 \\ SUMMARY, CONCLUSIONS AND FUTURE WORK}

\subsection{SUMMARY AND CONCLUSIONS}

Although the likelihood of using nanosecond lasers for micro machining has been predicted for some time, industrial application of this technique is awaited due to the challenges faced in terms of laser parameters and inadequate experimental studies in this area. The objective of this report was to further extend the capability of nanosecond laser for direct nanopatterning and overcome some challenges faced by the conventional techniques which currently in use in different industries.

First, a common path optical configuration was developed for nanograting impress based on a custom made prism. The silent feature of the configuration is that it is self aligned and has wide incident angle. With this optics, nanograting and nanodot array were generated in silicon substrates and gold thin films.

The experimental results outlined in Chapter 5 for direct fabrication of grating in silicon using fundamental harmonic wavelength of $1064 \mathrm{~nm} \mathrm{Nd:} \mathrm{YAG} \mathrm{laser} \mathrm{interference} \mathrm{was}$ demonstrated. Appropriate gratings period (d) of $1300 \mathrm{~nm}$ with high smoothness were successfully fabricated in silicon using a variety of exposure conditions and parameters mainly pulse repetition rate and dwell time. Study the influence of pulse energy, low repetition rate of $10-70 \mathrm{KHz}$ using low power nanosecond laser on grating patterns. Gratings were obtained by single shot of multi pulse exposures, and their characters were characterized using (SEM) measurements. 
Chapter 6 displays developing a new method for the generation of nanodot array and investigate phenomenon of ripple formation that associated with grating writing. The dot array was governed and arose from intersect two patterns, first is grating that generated by direct interference writing formula and second is ripple that generated by interference between the incident laser and the (FCW). Since the dot structures were defined by two kinds of mechanism formation which performed approximately at same time. The experimental results outlined in this Chapter demonstrate principally the influence of the fixed pulse width of $20 \mathrm{~ns}$, repetition rate, pulse energy, and the dwell time on the dots array formation as well as the dots separation created during irradiation.

In our experiments, the dots formed at a laser fluence intensity of $0.129 \mathrm{Jcm}^{-2}$. In the nanosecond regime, the dots as well as ripple formation on the silicon surface was based on the interference between the incident laser and the (FCW) mechanisms. The dimensions of an array due grating structuring depend upon the laser wavelength and the angle. The spacing between two adjacent elements is usually close to wavelength, it was $1300 \mathrm{~nm}$. While the dots separation $(\Lambda)$ by $(\mathrm{FCW})$ etching in a shallow liquid layer depends on laser fluency and pulse duration, it was $890 \mathrm{~nm}$. Theoretically, it was seen that for pulse width (laser pulse duration) used, repetition rate and pulse energy; the dot array spacing $(\Lambda)$ increased with the life time of the molten state $\tau_{L}$ that equal to laser pulse duration, as well as with effect of laser fluence Ǿ, while other parameters did not play as decisive a role.

Also, in Chapter 6, the secondary ripple formation on silicon surface which based on the interference between the incident laser and the (FCW) mechanisms have been demonstrated. At $30 \mathrm{KHz}$ pulse repetition rate with $0.015 \mathrm{~J} / \mathrm{cm}^{2}$ beam energy fluence measured $270 \mathrm{~nm}$ in ripple 
spacing $(\Lambda)$ for the fundamental wavelength of $1064 \mathrm{~nm}$. It was found that laser fluence and pulse width only played a significant role in the amount of ripples separation.

The experimental results outlined in Chapter 7 demonstrate that low pulse repetition rate range is ideal for surface patterning of silicon. It was seen that smaller wavelength lead to smaller grating period and higher repetition rate lead to uniform line width and high aspect ratio. Moreover, fabrication applying common optical path setup configuration using (SHG) wavelength of $532 \mathrm{~nm}$ of long pulse nanosecond laser as well as short pulse femtosecond laser of SHG wavelength of $400 \mathrm{~nm}$ provides more accurate and precise results which inspected by employing (SEM) and (AFM) measurements. It was found that pulse repetition rate only played a significant role in the size of the (HAZ) with femtosecond laser in the lower pulse energy ranges.

In conclusion, the principal achievements of the work described in this thesis are three, (i) the demonstration of interferometric Nd: YAG nanosecond laser fabrication for surface patterning of grating channels and dot array in semiconductor and metallic materials, (ii) the realization the efficiency of using nanosecond laser system in common optical path of simplest and fixed setup using custom-made prism for getting beams interference and (iii) An efficient and yet simple experimental setup was arrived at after studying the capabilities of the proposed laser system using a low repetition rate nanosecond laser while keeping the objectives of the research in mind. 


\subsection{FUTURE WORK}

The laser system used in these experiments does have many deficiencies especially for the type of economic basic laser system was used. The laser used has a maximum average power of $10 \mathrm{~W}$. In the experiments maximum power used was $8.80 \mathrm{~W}$ at $70 \mathrm{KHz}$ to get pulse energy of $0.125 \mu \mathrm{J}$, which inefficient for machining in range of nanosecond laser system at high repetition rate. As a result, all experiment results suffered from power fluctuation as evident in the (SEM) images. These applications would require a laser system with a higher maximum average power of approximately $15 \mathrm{~W}$ to obtain stable and enough pulse energy at higher repetition rate which lead to uniform line width and better pattern quality. Such a system would be capable of larger pulse energy increments allowing for more precise control of feature size. Future research should utilize a $15 \mathrm{~W}$ laser system to repeat the study in Chapters 5,6 and 7 in order to further optimize the repetition rate that would create the minimal feature size with enough laser energy. Using this kind of setup along with techniques such as different polarizations and a different gas assist may enable us to create sub $100 \mathrm{~nm}$ feature size with good quality and higher aspect ratio with minimal of HAZ effect. In the experiments outlined in Chapter 7, the (SHG) of $532 \mathrm{~nm}$ central wavelength was used. As a future work, these experiments can be performed at lower wavelength frequencies of $3^{\text {rd }}$ and $4^{\text {th }}$ harmonics generation or smaller. Fabrication use smaller wavelength frequencies for smaller features size and higher power laser. Even with the loss of laser power associated with the optics absorption and material reflection, this kind of setup may be able to further improve the quality of the machining as well as further reduce the size of the submicron features on the silicon wafer and thin gold film. Finally, use other material and study the effect of laser parameters as well as extend study more into femtosecond laser system. 


\section{REFERENCES}

[1] M. Kohler and W. Fritzsche, Nanotechnology: An Introduction to Nanostructuring Techniques, Weinheim, Germany: Wiley-VCH, $2^{\text {nd }}$ Edition, 2007

[2] M. C. Hutley, "Diffraction Gratings: Techniques of Physics": 6, London NW1, Great Britain: Academic press Inc., 1st Edition, 1982

[3] Maximilian N. Schulz, Progress in Optics Research, New York, USA, Nova Science Publishers, Inc. $1^{\text {st }}$ Edition, 2009

[4] Carol I. H. Ashby, Pattern formation techniques in photochemical etching, Thin Solid Films, 218 252-258 (1992)

[5] R. Fabian Pease, Lithography and Other Patterning Techniques for Future Electronics, IEEE Vol. 96, No. 2, February 2008

[6] M.S. Amera, M.A. El-Ashrya, L.R. Dosserb, K.E. Hixb, J.F. Maguirec and Bryan Irwin, Femtosecond versus nanosecond laser machining: comparison of induced stresses and structural changes in silicon wafers, Applied Surface Science 242 (2005) 162-167

[7] B.N. Chichkov, C. Momma, S. Nolte, F. von Alvensleben, A. Tu-nnermann, "Femtosecond. picosecond and nanosecond laser ablation of solids", Appl. Phys. A 63, 109-115 (1996)

[8] Pronko, P.P., Dutta, S.K., Squier, J., Rudd, J.V., Du, D., Mourou, G., Thermophysical Effects in Laser Processing of Materials with Picosecond and Femtosecond Pulses, Journal of Applied Physics 78.10 (1995): $6233-40$

[9] Gamaly, E.G., Rode, A.V., Luther-Davies, D., Ultrafast Ablation with High-Pulse-Rate Lasers. Part I: Theoretical Considerations, Journal of Applied Physics 85.8 I (1999): 4213-21

[10] Bonse, J., Baudach, S., Kru $\square$ ger, J., Kautek, W., Lenzner, M., Femtosecond Laser Ablation of Silicon-Modification Thresholds and Morpholog, Applied Physics A: Materials Science and Processing 74.1 (2002): 19-25 
[11] Scha $\square$ fer, C., Urbassek, H. M., Zhigilei, L. V., Metal Ablation by Picosecond Laser Pulses: A Hybrid Simulation, Physical Review B - Condensed Matter and Materials Physics 66.11 (2002): $1154041-8$

[12] Lorazo, P., Lewis, L. J., Meunier, M., Short-Pulse Laser Ablation of Solids: From Phase Explosion to Fragmentation, Physical Review Letters 91.22 (2003)

[13] Hardway, GA, Applications of Laser Systems to Microelectronics and Silicon Wafer Dicing, Solid State Technology 13.4 (1970): 63-7

[14] Kolomenskii, Al A., et al, Interaction of Laser-Generated Surface Acoustic Pulses with Fine Particles: Surface Cleaning and Adhesion Studies, Journal of Applied Physics 84.5 (1998): 24042410.

[15] Henry, M., J. Wendland and P. M. Harrison, Nanoscale Analysis of Laser Ablated Thin Films used in Industrial Manufacturing of Flat Panel Displays, ICALEO (2006) - 25th International Congtess on Applications of Laser and Electro-Optics, Congress Proceedings

[16] Leiderer, P., et al, Laser-Induced Particle Removal from Silicon Wafers, Proceedings of SPIE - The International Society for Optical Engineering, (2000), 4065, 249-259

[17] Clark-MXR Inc., Dexter, MI, USA, (Feb. 4, 2008) http://www.cmxr.com/Industrial/Handbook

[18] Yi-Jie Jia, J., "The Mechanism of Thin Film Si Nanomachining Using Femtosecond Laser Pulses", Master of Science Thesis, June 2004, Massachusetts Institute of Technology (MIT)

[19] Liau, Z. L., et al, Reduction of Thermal Conductivity in Wafer-Bonded Silicon, Applied Physics Letters 93.2 (2008)

[20] Liu X, Du D, Mourou G., "Laser ablation and micromachining with ultrashort laser pulses", IEEE J. Quantum Electron, Vol. 33, No. 10, 1706-1716, 1997

[21] Kononenko, T. V., et al. "Comparative Study of the Ablation of Materials by Femtosecond and Pico-Or Nanosecond Laser Pulses", Quantum Electronics 29.8 (1999): 724-8

[22] Le Harzic, R., et al. "Comparison of Heat-Affected Zones due to Nanosecond and Femtosecond Laser Pulses using Transmission Electronic Microscopy", Applied Physics Letters 80.21 (2002): 3886 
[23] Lee, B. M., et al. "Molecular-Dynamics Analysis of the Nucleation and Crystallization Process of Si", Physica B: Condensed Matter 392.1-2 (2007): 266-71

[24] Bonse, J., K. -W Brzezinka, and A. J. Meixner. "Modifying Single-Crystalline Silicon by Femtosecond Laser Pulses: An Analysis by Micro Raman Spectroscopy, Scanning Laser Microscopy and Atomic Force Microscopy", Applied Surface Science 221.1-4 (2004): 215-30.

[25] Pronko, P. P., et al. "Machining of Sub-Micron Holes using a Femtosecond Laser at $800 \mathrm{Nm}$ ", Optics Communications 114.1-2 (1995): 106-10

[26] Ermer, D. R., et al, "Ablation of Dielectric Materials during Laser Irradiation Involving Strong Vibrational Coupling", Applied Physics A: Materials Science and Processing 70.6 (2000): 633-5.

[27] Takeshima, M. "Auger Recombination in a Quasi-One-Dimensional-Structure Semiconductor", Physical Review B 31.2 (1985): 992-9

[28] Burakov, I. M., et al. "Theoretical Investigations of Material Modification using Temporally Shaped Femtosecond Laser Pulses", Applied Physics A: Materials Science and Processing 81.8 (2005): 1639-45

[29] Hirayama, Y., and M. Obara. "Heat Effects of Metals Ablated with Femtosecond Laser Pulses", Applied Surface Science_197-198 (2002): 741-5

[30] Van Vechten, J. A., et al. "Reasons to Believe Pulsed Laser Annealing of Si does Not Involve Simple Thermal Melting", Physics Letters A_74.6 (1979): 417-21

[31] Van Vechten, J. A., R. Tsu, and F. W. Saris, "Non-thermal Pulsed Laser Annealing of Si; Plasma Annealing", Physics Letters A74.6 (1979): 422-426

[32] Sokolowski-Tinten, K., et al. "Transient States of Matter during Short Pulse Laser Ablation", Physical Review Letters_81.1 (1998): 224-227

[33] Cavalleri, A., et al. "Femtosecond Melting and Ablation of Semiconductors Studied with Time of Flight Mass Spectroscopy", Journal of Applied Physics_85.6 (1999): 3301-9.

[34] Izawa, Y., et al. "Ultrathin Amorphous Si Layer Formation by Femtosecond Laser Pulse Irradiation", Applied Physics Letters 90.4 (2007). 
[35] Agranat, M. B., S. I. Anisimov, and B. I. Makshantsev. "The Anomalous Thermal Radiation from Metals Produced by Ultrashort Laser Pulses", Applied Physics B Photophysics and Laser Chemistry 47.3 (1988): 209-21

[36] Lu, Q., et al. "Theory Analysis of Wavelength Dependence of Laser-Induced Phase Explosion of Silicon", Journal of Applied Physics_104.8 (2008)

[37] Kaebernick, H., D. Bicieanu, and M. Brandt. "Theoretical and Experimental Investigation of Pulsed Laser Cutting", CIRP Annals - Manufacturing Technology_48.1 (1999): 163-6

[38] Wang, X. Y., et al. "Time-Resolved Electron-Temperature Measurement in a Highly Excited Gold Target using Femtosecond Thermionic Emission", Physical Review B_50.11 (1994): 8016-9

[39] Fann, W. S., et al. "Electron Thermalization in Gold", Physical Review B_46.20 (1992): 13592-5

[40] Preuss, S., A. Demchuk, and M. Stuke. "Sub-Picosecond UV Laser Ablation of Metals", Applied Physics A Materials Science \& Processing_61.1 (1995): 33-37

[41] M. von Allmen, A. Blatter, "Laser-Beam Interactions with Materials", Springer-Verlag, Berlin 1995

[42] Herziger, G., Kreutz, E. W., Wissenbach, K., "Fundamentals Of Laser Processing Of Materials", Proceedings of SPIE - The International Society for Optical Engineering, (1986), 668, 2-10

[43] B Tan and K Venkatakrishnan, "A femtosecond laser-induced periodical surface structure on crystalline silicon", J. Micromech. Microeng. 16 (2006) 1080-1085

[44] C.W. Luo, C. C. Lee, C. H. Li, H. C. Shih, Y.-J. Chen, C. C. Hsieh, C. H. Su, W. Y. Tzeng, K. H.Wu, J. Y. Juang, T. M. Uen, S. P. Chen, J.-Y. Lin and T. Kobayashi, "Ordered YBCO submicron array structures induced by pulsed femtosecond laser irradiation", OPTICS EXPRESS 20610, Vol. 16- No. 25, 8 Dec 2008

" [45] Klein-Wiele, J.H. and Simon P., "Enhancement of laser nano-patterning of semiconductors: Direct ablation of PMMA coated silicon", Conference on Lasers and Electro-Optics and 2006 Quantum Electronics and Laser Science Conference, CLEO/QELS 2006 Volume , Issue , 21-26 May 2006 Page(s):1 - 2 
[46] J.H. Klein-Wiele, J. Bekesi and P. Simon, "Sub-micron patterning of solid materials with ultraviolet femtosecond pulses", Appl. Phys A 79, 775-778 (2004)

[47] B. N. Chichkov, J. Koch, A. Ovsianikov, S. Passinger, C. Reinhardt and J. Serbin, "Direct-Write Micro- and Nanostructuring with Femtosecond Lasers", Materials Research Society Symposium Proceedings, vol. 850, pp. 179-186, 2005

[48] K. Venkatakrishnan, N. R. Sivakumar and B. Tan, "Fabrication of Planar Grating by direct ablation using Ultrashort Pulse Laser in a common optical path configuration", Applied Physics A, Vol 76, $143-146,2002$.

[49] Krishnan Venkatakrishnan, B. Tan, Paul Stanley, L. E. N. Lim and Bryan Kok Ann Ngoi, Opt. Eng. 41(6) 1441-1445 (June 2002)

[50] Nolte, S., Momma, C., Kamlage, G., Chichkov, B.N., Tuennermann, A., von Alvensleben, F., Welling, H., "Micromachining with Femtosecond Lasers." Conference on Lasers and ElectroOptics Europe - Technical Digest", (1998), 510-511

[51] Chang, J.J., Warner, B. E., "Laser-Plasma Interaction during Visible-Laser Ablation of Methods", Applied Physics Letters 69.4 (1996): 473-475

[52] Graham D. Marshall, Optics Letter, Vol. 31, No. 18, September 15, 2006

[53] T. T. Chen and Da H-Min Hawang, Chinese journal of Physics Vol. 15, No. 1 Spring, 1977

[54] Katsuhiro Munakata, Kenji Harada, Nobukazu Yoshikawa, Masahide Itoh, Shinsuke Umegaki and Toyohiko Yatagai 0-7803-5661-6/1999 IEEE, 98 /CLEO/Pacific Rim 99/TuL4

[55] K. Venkatakrishnan, N.R. Sivakumar, C. W. Hee, B. Tan, W. L. Liang and G. K. Gan, Applied Physic A, Volume 77, Number 7,959-963, 2003

[56] Csaba Vass and Karoly Osvay, 4 September $2006 /$ Vol. 14, No. $18 /$ optics express 8354 "Fabrication of $150 \mathrm{~nm}$ period grating in fused silica by two-beam interferometric laser induced backside wet etching method"

[57] Bo Tan, Narayanswamy R Sivakumar and Krishnan Venkatakrishnan, J. Opt. A: pure Appl. Opt. 7 (2005) 169-174 
[58] Yokotani, A., Matsuo, N., Kawahara, K., Kurogi, Y., Matsuo, N., Ninomiya, T., Sawada, H., Kurosawa, K. , "Development of Dicing Technique for Thin Semiconductor Substrates with Femtosecond Laser Ablation", Proceedings of SPIE - The International Society for Optical Engineering, (2002), 4637, 180-187

[59] Kamata, M., Imahoko, T., Ozono, K., Obara, M. "Materials Processing by use of a Ti: Sapphire Laser with Automatically-Adjustable Pulse Duration", Applied Physics A: Matcrials Science and Processing 79.7 (2004): 1679-1685

[60] Bliss, D.E., Adams, D.P., Cameron, S.M., Luk, T.S., "Laser machining with ultrashort pulses: Effects of pulse-width, frequency and energy" Materials Research Society Symposium Proceedings 546, (1999): 81-84

[61] To $\square$ nshoff, H.K., Ostendorf, A., Korte, F., Serbin, J., Bauer, T., "Generation of Periodic Microstructures with Femtosecond Laser Pulses", Second International Symposium on Laser Precision Microfabrication. Singapore, (2001)

[62] D.Bauerle, Journal of Photochemistry and Photobiology a: Chemistry 106 (1997) 27-30

[63] Anant Chimmalgi, David J. Hwang and Costas P. Grigoropoulos, Nano Letters, Vol. 5, No. 10, 1942-1930, (2005)

[64] Hong-Yu Chu, M.S. Thesis, Department of Physics National Central University (May, 2002)

[65] P. Ecija et al, physical review A 77, 032904, 2008

[66] D. Ivanov et al, G. O'Connor et al, and A. Volkov et al, "The mechanism of nano bump formation in short pulse laser nanostructuring of metals", PACS: 61.80.Az, 02.70.Ns, 64.70.D

[67] Jing Huang, Yuwen Zhang *, J.K. Chen, "Ultrafast solid-liquid-vapor phase change in a thin gold film irradiated by multiple femtosecond laser pulses", International Journal of Heat and Mass Transfer 52 (2009) 3091-3100

[68] K. Nishioka and S. Horita, "Periodic arrays of submicron $\mathrm{Si}$ and Ni dots on SiO2 fabricated using linearly polarized Nd: YAG pulsed laser", Appl. Phys: A 91, 235-240 (2008)

[69] D. Kawamura, A. Takita, Y. Hayasaki, and N. Nishida, "Bump formation on a glass surface with a transparent coating using femtosecond laser processing", Appl. Phys: A 85, 39-43 (2006) 
[70] D. Mills, T. Kreouzis, A. Sapelkin, B. Unal, N. Zyuzikov, K.W. Kolasinski, "Surface texturing of Si, porous Si and TiO2 by laser ablation"

[71] Zhongyi Guo, Shiliang Qu, Lingling Ran, Yanhua Han, and Shutian Liu, "Formation of twodimensional periodic microstructures by a single shot of three interfered femtosecond laser pulses on the surface of silica glass", Optics Letters Vol. 33, No. 20 (2008)

[72] Jinhai Si , Zhichong Meng, Shingo Kanehira, Jianrong Qiu, Bin Hua and Kazuyuki Hirao, , "Multiphoton-induced periodic microstructures inside bulk azodye-doped polymers by multibeam laser interference", Chemical Physics Letters 399 (2004) 276-279

[73] Q. Zhao, J. Qiua, C. Zhao, X. Jiang and C. Zhu, "Formation of array microstructures on silicon by multi-beam interfered femtosecond laser pulses" , Applied Surface Science 241 (2005) 416-419

[74] P.L. Liu, R.Yen, N. Bloembergen and R.T. Hodgson, "Picosecond laser-induced melting and resolidification morphology on Si", Applied Physics Letters, Vol. 34, No. 12 (1979), pp. 864-6

[75] S.I. Dolgaev, S.V. Lavrishev, A.A. Lyalin, A.V. Simakin, V.V. Voronov, G.A. Shafeev, "Formation of conical microstructures upon laser evaporation of solids", Appl. Phys. A 73, 177$181(2001)$

[76] V.I. Emel'yanov, D.V. Babak, "Defect capture under rapid solidification of the melt induced by the action of femtosecond laser pulses and formation of periodic surface structures on a semiconductor surface", Appl. Phys: A 74, 797-805 (2002)

[77] Y. F. Lua, W. K. Choi, Y. Aoyagi, A. Kinomura and K. Fujii, "Controllable laser-induced periodic structures at silicon-dioxide/silicon interface by excimer laser irradiation", Appl. Phys: 80, 12 (1996)

[78] Brijesh Kumar and R K Soni, "Sub-micrometer periodic surface structures in InP induced by nanosecond UV laser pulses", Appl. Phys. 41 (2008) 155303 (6pp)

[79] K. Venkatakrishnan, P. Stanley, N.R. Sivakumar, B. Tan and L.E.N. Lim, "Effect of scanning resolution and fluence fluctuation on femtosecond laser ablation of thin films", Appl. Phys A: Materials Science and Processing, Vol. 77 No. 5, 655-658, (2003) 
[81] The Institution of Electrical Engineers, London, United Kingdom, 1999, "Properties of Crystalline Silicon", Published by: INSPEC, the Institution of Electrical Engineers, page 157, ISBN 085296 933

[82] C. Wu, C.H. Crouch, L. Zhao, E. Mazur, Appl. Phys. Letter. 81 (2002) 1999

[83] G. Gorodetsky, J.Kanicki, T. Kazyaka, and R.L.Melcher, "Far UV Laser Melting of Silicon", Applied Physics Letters, Vol. 46, No. 6 (1985), 547-549

[84] T.R. Anthony and H.E. Cline, "Surface rippling induced by surface-tension gradients during laser surface melting and alloying", Journal of Applied Physics, Vol. 48, No. 9 (1977), pp. 3888-94

[85] D. Jost, W.Luthy, H.P.Weber and R.P.Salathe, "Laser pulse width dependent surface ripples on silicon", Applied Physics Letters, Vol. 49, No. 11 (1986), pp. 625-627

[86] J. Bonse, M. Munz and H. Sturm, "Structure formation on the surface of indium phosphide irradiated by femtosecond laser pulses", Journal of applied physics 97, 013538 (2005)

[87] Mizunori Ezaki, Hiroshi Kumagai, Koichi Toyoda, and Minoru Obara, "Surface Modification of 111-V Compound Semiconductors Using Surface Electromagnetic Wave Etching Induced by Ultraviolet Lasers", IEEE Journal of selected topics in quantum electronics, Vol. 1, No. 3, Sep. 1995

[88] Nastaran Mansour, Kazem Jamshidi-Ghaleh, and David Ashkenasi, "Formation of Conical Microstructures of Silicon with Picosecond Laser Pulses in Air", JLMN-Journal of Laser Micro/Nanoengineering, Vol. 1, No. 1, 2006

[89] M.S. Trtica, B.M. Gakovic, B.B. Radak, D. Batani, T. Desai, M. Bussoli, "Periodic surface structures on crystalline silicon created by $532 \mathrm{~nm}$ picosecond Nd: YAG laser pulses", Applied Surface Science 254 (2007) 1377-1381

[90] N.B. Dahotre and S.P. Harimkar, "Laser Fabrication and Machining of Materials", Springer 2008, ISBN 978-0-387-72343-3

[91] Max Born and Emil Wolf, "Principles of optics: electromagnetic theory of propagation", 7th edition, 1999, ISBN 0521642221

[92] Christopher Palmer, "diffraction grating handbook", sixth edition, Newport Corporation, Erwin Loewen, first edition, New York 14605 USA

[93] http://mysite.du.edu/ etuttle/optics/ywave.htm\#Oth 
[94] O. I. Szentesi and E. A. Noga, "Parylene C Films for Optical Waveguides", Appl. Opt. 13, 24582459 (1974)

[95] MNT Bulletin Vol.9/No.1 - 2008, "Development of Lithography Technology for Nanoscale Structuring of Materials Using Laser Beam Interference", "ttp://www.imt.ro/mnt/V9N1/pag\%208.pdf"

[96] M. Schnieper, M. T. Gale, C. Zschokke, F. Di Prima, B. Satilmis and C. David, "Grating nanostructures for optical elements", CSEM science and technology report 2002, 30

[97] M. Schnieper, M. T. Gale, B. Satilmis, J. Sochtig, C. Zschokke, and C. David, "Subwavelength optics", CSEM science and technology report 2001, 24

[98] M. C. Castex, A. Fischer, D. Ades and A. Siove, "Fabrication of polymer surface relief gratings with UV and VUV lasers", The 7th International Conference on Laser Ablation, October 5-10, 2003, Hersonissos, Crete, Greece

[99] Janne Simonen, "Applied diffractive optics with electron beam lithography", Dissertations 37, Department of physics, University of Joensuu

[100] Zhaoning Yu, Paru Deshpande, Wei Wu, Jian Wang, and Stephen Y. Chou, "Reflective polarizer based on a stacked double-layer subwavelength metal grating structure fabricated using nanoimprint lithography", Applied Physics Letters, 77 (7): 927-929, 2000.

[101] K. Venkatakrishnan, B. Tan, and B. K. A. Ngoi, "Submicron Holes in Copper Thin Film Directly Ablated Using Femtosecond Pulsed Laser", Optical Engineering, Vol.40, No.12, 2892-2893, Dec. 2001

[102] K. Kawamura, N. Sarukura, M. Hirano and H. Hosono, "Holographic encoding of permanent gratings embedded in diamond by two beam interference of a single femtosecond near infrared laser pulse", Japanese Journal of Applied Physics, 39, L767-769, 2000

[103] Eunha Kim, Digant Dave and Thomas E. Milner, "Fiber-optic spectral polarimeter using a broadband swept laser source", Optics Communications, Volume 249, Issues 1-3, 1 May 2005, Pages 351-356 